\title{
O problema do centro-foco para singularidades nilpotentes no plano
}

\author{
Jackson Itikawa
}



SERVIÇO DE PÓS-GRADUAÇÃO DO ICMC-USP

Data de Depósito:

Assinatura:

\title{
O problema do centro-foco para singularidades nilpotentes no plano
}

\author{
Jackson Itikawa
}

Orientadora: Profa. Dra. Regilene Delazari dos Santos Oliveira

Dissertação apresentada ao Instituto de Ciências Matemáticas e de Computação - ICMC-USP, como parte dos requisitos para obtenção do título de Mestre em Ciências - Matemática . EXEMPLAR DE DEFESA

USP - São Carlos

Março de 2012 
Ficha catalográfica elaborada pela Biblioteca Prof. Achille Bassi e Seção Técnica de Informática, ICMC/USP, com os dados fornecidos pelo(a) autor(a)

Itikawa, Jackson
O problema do centro-foco para singularidades
nilpotentes no plano / Jackson Itikawa; orientadora
Regilene Delazari dos Santos Oliveira. -- São
Carlos, 2012 .
126 p.
Dissertação (Mestrado - Programa de Pós-Graduação en
Matemática)-- Instituto de Ciências Matemáticas e
de Computação, Universidade de São Paulo, 2012.
1. Problema do centro-foco. 2. Singularidades
nilpotentes. 3. Constantes de Lyapunov. 4.
Monodromia. I. Delazari dos Santos Oliveira,
Regilene, orient. II. Título.


Aos pilares da minha vida:

Judith, Katuhide, Katielly e Mauricio. 


\section{Agradecimentos}

A produção intelectual, sobretudo a acadêmica, costuma ser apresentada em seu formato final, acabado. Oculta-se, assim, a falta de inspiração, a síndrome da folha branca, os momentos de lassidão e fadiga, e mesmo os instantes de inspiração fulgurante, que resultam na solução de um problema difícil ou no vislumbre de uma demonstração há muito procurada.

Neste trabalho não foi diferente, e tenho certeza que não o será em outros que virão. Mas é inegável a sua importância em minha vida. É o resultado de escolhas que fiz ao longo desses anos, algumas delas bastante difíceis; é o ápice de muitas conquistas que me exigiram grande esforço e muita força de vontade. Não me arrependo de minhas escolhas e orgulho-me de minhas conquistas. Acima de tudo, sou grato pelo afeto, apoio, incentivo e compreensão que recebi ao longo desse tempo, sem os quais certamente não poderia ir tão longe. Chego até aqui com o sentimento de dever cumprido, mas não satisfeito. Mais escolhas e conquistas ainda estão por vir e quero sempre ir além.

Agradeço à minha mãe, Judith, e ao meu pai, Katuhide, por todo afeto, confiança, respeito e dedicação. Pelo exemplo de coragem, força, humildade e determinação.

Atribuem a Bertrand Russell uma frase maravilhosa: "os nossos pais amam-nos porque somos seus filhos, é um fato inalterável. Nos momentos de sucesso, isso pode parecer irrelevante, mas nas ocasiões de fracasso, oferecem um consolo e uma segurança que não se encontram em qualquer outro lugar." Obrigado pai e mãe por sempre acreditarem em mim, mesmo quando eu mesmo deixei de acreditar!

À minha irmã Katielly, tão mais jovem que eu, mas tão mais madura e sábia. Dona de um coração abnegado e afetuoso, a quem confio minhas maiores tristezas e compartilho sempre as grandes e pequenas alegrias. 
Ao meu irmão Mauricio, que, com seu jeito reservado e mesmo morando tão longe, está sempre zelando pelo bem estar da família. Quando precisei, não hesitou. Largou tudo e foi em meu socorro.

À professora Regilene, pela competência, amizade e confiança. Sua mão segura me guiou nos primeiros passos da pesquisa acadêmica com maestria, dedicação e paciência.

Nesses anos todos, tive a felicidade de encontrar algumas pessoas especiais, amigos para a vida inteira. Sou-lhes muito grato pelo apoio e amizade. Agradeço especialmente ao Marco Queiroz e à Liane Weismann, por seu afeto, sabedoria e disponibilidade para me ouvir; ao Caio Areias, pelas intermináveis conversas, muitas delas discussões acaloradas que invariavelmente terminavam em boas risadas; à Irene Carvalho, amiga fiel de tantos anos; à Noemi Rocha, minha grande companheira nesses anos todos no ICMC; à Adriana Pereira Gomes, uma das almas mais generosas que já conheci; ao Silas Vaz da Silva, por me ensinar o valor da ética profissional e pela amizade duradoura; à Leila Karina (in memoriam), cujo afeto e alegria estão indeléveis em meu coração.

Aos professores e mestres Paulo "Jacaré" Almeida, Cláudia Cueva Cândido, João Eduardo Kogler Jr., José Jaime Cruz e Maria Aparecida Soares Ruas, pelo incentivo, apoio e exemplo de dedicação e competência. Cada um a seu modo, fez crescer em mim o gosto pela pesquisa e o amor à Matemática.

Aos professores do ICMC, sou grato pela rica experiência de aprendizado; à Regina Medeiros e à Irene Lucinda, que sempre me atendem com cordialidade e eficiência na biblioteca do ICMC; à Ana Paula Fregona da secretaria da pós-graduação, por sua solicitude e paciência. Aos funcionários do ICMC, atenciosos e colaboradores.

À Ingrid Sofia Mesa e ao Alex Rezende, bons companheiros de pesquisa. Aos amigos que fiz em minha graduação e durante o mestrado, com quem dividi intermináveis horas de estudo, mas também bons momentos de descontração. Pelo apoio, aprendizagem, paciência e amizade, muito obrigado!

Ao professor Ricardo Miranda Martins, pela grande colaboração no desenvolvimento das planilhas do Maple ${ }^{\circledR}$ usadas neste trabalho.

À FAPESP, pela bolsa que possibilitou esta pesquisa.

Enfim, a todos aqueles que colaboraram de alguma forma com esta dissertação, meus sinceros agradecimentos. 
"Porque tenho sido tudo, e creio que minha verdadeira vocação é procurar o que valha a pena ser."

- Monteiro Lobato 



\section{Resumo}

O estudo dos pontos singulares em campos vetoriais analíticos é um problema quase completamente resolvido. O único caso que ainda permanece insolúvel é o caso monodrômico, em que as órbitas circundam a singularidade. Em sistemas diferenciais analíticos, se $p$ é singularidade monodrômica, então $p$ ou é um centro, ou é um foco. O problema do centro-foco consiste em determinar condições que diferenciem os casos em que $p$ é um foco, daqueles em que $p$ é um centro.

O tema central desta dissertação é a investigação do problema do centro-foco em sistemas diferenciais analíticos com singularidade nilpotente. Este problema é bastante estudado, uma vez que ainda não existe um algoritmo eficiente para este caso, tal como ocorre em sistemas com singularidades não degeneradas.

Estudamos duas técnicas bastante distintas. A primeira faz uso da teoria das formas normais e aborda o problema da maneira clássica, dividindo-o na investigação da monodromia e no estudo da estabilidade. O outro método investiga os sistemas diferenciais com singularidades nilpotentes como limite de sistemas com singularidades não degeneradas.

A fim de avaliarmos sua eficiência e compreendermos as possíveis obstruções envolvidas, aplicamos os métodos a famílias concretas de sistemas diferenciais.

Palavras-chave: Problema do Centro-Foco; Constantes de Lyapunov; Singularidades Nilpotentes; Monodromia. 


\section{Abstract}

The study of singular points in planar analytic vector fields is a problem almost completely solved. The only case that remains open is the monodromic one, in which the orbits turn around the singularity. In analytic differential systems, if $p$ is a monodromic singular point, then $p$ is either a center or a focus. The center-focus problem consists in determining conditions for distinguishing between a center and a focus.

The main purpose of this work is the investigation of the center-focus problem in analytic differential systems with nilpotent singular points. This problem is still widely studied, since there is no algorithm for such case, comparable to the Lyapunov method for the case of non-degenerate singularities.

We studied two different methods. The first makes use of the normal form theory and deals with the problem in the classic way, splitting it up in two parts: the investigation of the monodromy and the study of the stability. The latter investigates the differential analytic systems with nilpotent singular points as limit of differential systems with nondegenerate singularities.

In order to evaluate the efficiency and understand possible obstructions, we applied the two techniques to concrete families of differential systems.

Key words: The Center-Focus Problem; Lyapunov Constants; Nilpotent Singularities; Monodromy. 



\section{Sumário}

1 Elementos da teoria qualitativa das EDO's no plano 5

1.1 Campos vetoriais e pontos singulares . . . . . . . . . . . . 5

1.2 Classificação local de campos de vetores . . . . . . . . . . . . . . . . . 10

1.3 Formas normais . . . . . . . . . . . . . . . . . . . . 17

1.4 Desingularização de pontos singulares . . . . . . . . . . . . . . . . 20

2 Problema do centro-foco $\quad 37$

2.1 Singularidades não degeneradas . . . . . . . . . . . . . . . . . 37

2.1.1 Preâmbulo teórico . . . . . . . . . . . . . . . . . . . . 38

2.1.2 Constantes de Lyapunov . . . . . . . . . . . . . . . . . . . . 55

2.1 .3 Algoritmo de Lyapunov . . . . . . . . . . . . . . . . 57

2.1.4 Campos vetoriais quadráticos no plano . . . . . . . . . . . . . 62

2.2 Singularidades degeneradas linearmente nulas . . . . . . . . . . . . 66

3 Problema do centro-foco: singularidades nilpotentes $\quad 71$

3.1 Método de Álvarez-Gasull . . . . . . . . . . . . . . . . . . . . . . . 71

3.1.1 Problema da monodromia . . . . . . . . . . . . . . . . 72

3.1.2 Constantes de Lyapunov generalizadas . . . . . . . . . . . . . . 82

3.1.3 Problema da estabilidade . . . . . . . . . . . . . . . . . 84

3.2 Método de Giacomini-Giné-Llibre . . . . . . . . . . . . . . . . . . 97 
4.1 Método de Álvarez-Gasull . . . . . . . . . . . . . . . . . . . . . . . . . . . 104

4.2 Método de Giacomini-Giné-Llibre . . . . . . . . . . . . . . . . . . 108

5 Conclusões

A Componentes da função $b(x)$ 


\section{Lista de Figuras}

1.1 Interpretação geométrica do teorema de Hartman-Grobman . . . . . . . . . . 12

1.2 Esboço do retrato de fase do campo $\hat{X} \ldots \ldots \ldots \ldots$

1.3 Exemplo de blow up polar . . . . . . . . . . . . . . . . 25

1.4 Exemplo de blow up direcional . . . . . . . . . . . . . . . . 28

1.5 Relação entre blow up na direção x e blow up polar . . . . . . . . . . . . . 29

1.6 Relação entre blow up na direção y e blow up polar . . . . . . . . . . . . . 30

1.7 Exemplo de blow ups direcionais sucessivos . . . . . . . . . . . . . . . 32

1.8 Exemplo de blow-up $(\mathrm{m}, \mathrm{n})$-quasehomogêneo $\ldots \ldots \ldots$. . . . . . . . . 34

2.1 Órbita periódica $\gamma_{0}$, curvas fechadas simples $\Gamma_{1}$ e $\Gamma_{2}$ e a região $\Omega$. . . . . 39

2.2 Interpretação geométrica da Prop. 2.1.1 para o sistema (2.0.1) . . . . . . . 44

2.3 Interpretação geométrica da Prop. $2.1 .10 \ldots \ldots$. . . . . . . . 54

3.1 Índice de um campo vetorial analítico com monodromia . . . . . . . . . . . 74 


\section{Introdução}

A caracterização local dos retratos de fase de campos vetoriais planares é um dos problemas clássicos da teoria qualitativa das equações diferenciais ordinárias (EDO's). Este problema está quase completamente resolvido, restando essencialmente o caso em que a singularidade não tem direções características (monodromia). Em sistemas diferenciais analíticos, se $p$ é monodrômico, então $p$ ou é um centro, ou é um foco - cf. [33].

Considere o sistema diferencial analítico no plano:

$$
\left\{\begin{array}{l}
\dot{x}=P(x, y), \\
\dot{y}=Q(x, y),
\end{array}\right.
$$

e seja $p$ um ponto singular isolado deste sistema.

O problema do centro-foco consiste em determinar condições que diferenciem os casos em que $p$ é um foco dos casos em que $p$ é um centro - cf. [28, 38].

A investigação do problema do centro-foco pode ser dividida em três casos, de acordo com o tipo de singularidade:

- Pontos singulares não degenerados: o problema do centro-foco foi teoricamente solucionado por Poincaré [45] e Lyapunov [36]. Entretanto, obstruções computacionais e de suficiência ainda não permitem obter uma solução geral para famílias concretas de sistemas diferenciais, à exceção dos campos vetoriais quadráticos de grau 2 - cf. [10, 21];

- Pontos singulares degenerados nilpotentes: neste caso, o problema do centrofoco usualmente é dividido em duas partes, o problema da monodromia, que foi 
solucionado por Andreev - cf. [3], em 1958, e o problema da estabilidade, resolvido por Moussu - cf. [41], em 1982. Tal como no caso anterior, obstruções computacionais e de suficiência dificultam a investigação de centros nilpotentes em sistemas diferenciais concretos. Este caso é um dos mais investigados até hoje, com grande número de artigos publicados a respeito - cf. [1, 2, 23, 25, 29];

- Pontos singulares degenerados com parte linear identicamente nula: ainda é um caso em aberto. Os resultados obtidos se referem a famílias específicas de sistemas diferenciais e acredita-se que uma solução geral ainda está longe de ser alcançada - cf. [28, 24, 29].

Nesta dissertação, vamos discutir o problema do centro-foco em sistemas com singularidades isoladas do tipo nilpotente, apresentando exemplos e discutindo as obstruções encontradas. Os demais casos também serão apresentados, porém, de maneira mais sucinta, com a apresentação de alguns resultados relevantes.

A dissertação está organizada da seguinte forma:

No CAPÍTULO 1, introduzimos notações, apresentamos definições básicas tais como seção transversal, monodromia, e a classificação de pontos singulares, dentre outros. Além disso, descrevemos as formas normais e técnicas de blow-up. O objetivo deste capítulo é fornecer as ferramentas necessárias ao desenvolvimento realizado nos capítulos posteriores.

O CAPÍTULO 2 é dedicado ao problema do centro-foco em sistemas diferenciais analíticos com singularidades não degeneradas e singularidades linearmente nulas. Apresentamos importantes conceitos e resultados, como as constantes de Lyapunov, ferramenta fundamental no estudo do problema do centro-foco em sistemas com pontos singulares não degenerados, e o Teorema de Bautin - cf. [10], que fornece solução para o problema do centro-foco em campos quadráticos planares. Alguns resultados referentes ao problema do centro-foco em sistemas com singularidades linearmente nulas também são brevemente discutidos.

O caso nilpotente é investigado em detalhes no CAPÍTULO 3, com a apresentação de dois métodos. A técnica desenvolvida por Álvarez \& Gasull - cf. [1, 2], que faz uso da teoria das formas normais e da técnica de blow-up (1,n)-quasehomogêneo, dentre outros, fornece demonstrações mais elegantes para os teoremas de Andreev - cf. [3] (problema da monodromia) e de Moussu - cf. [41] (problema da estabilidade). Outra abordagem, 
devida a Giacomini, Giné \& Llibre - cf. [29], utiliza a aproximação de sistemas diferenciais com centro nilpotente por sistemas com centros lineares.

No CAPÍTULO 4, as técnicas estudadas no terceiro capítulo são aplicadas em sistemas concretos, possibilitando avaliar sua eficácia, bem como identificar as dificuldades encontradas.

Ao final da dissertação, são feitas algumas considerações finais, e apresentadas as referências bibliográficas utilizadas na pesquisa. 


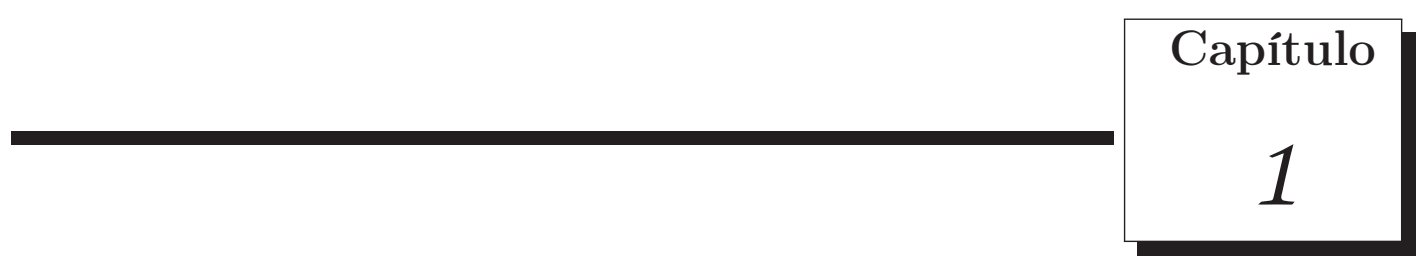

Elementos da teoria qualitativa das EDO's no plano

Nesse capítulo, apresentamos conceitos e resultados da teoria qualitativa das EDO's no plano, necessários para a investigação do problema do foco centro, tema dessa dissertação. Alguns tópicos são apresentados de maneira detalhada, enquanto em outros, fornecemos referências para maiores detalhes.

\subsection{Campos vetoriais e pontos singulares}

Definição 1.1.1. Seja $\Delta$ um subconjunto aberto de $\mathbb{R}^{n}$. Um campo vetorial de classe $C^{k}$ em $\Delta$ é uma aplicação $X: \Delta \rightarrow \mathbb{R}^{n}$, de classe $C^{k}$, onde $k$ é um inteiro positivo, $+\infty$, ou $\omega, e C^{\omega}$ denota o conjunto das funções analíticas.

As curvas $x=\varphi(t), t \in I \subset R$, que são soluções da equação diferencial

$$
\dot{x}=X(x)
$$

são chamadas de curvas integrais do campo vetorial $X$. Observe que em (1.1.1), $x \in \Delta$ e $\dot{x}:=d x / d t$, As variáveis $x$ e $t$ são chamadas respectivamente de variável dependente 
e variável independente da equação diferencial. Usualmente, $t$ é designado como tempo. No caso em que $X=X(x)$ independe de $t$, dizemos que a equação diferencial (1.1.1) é autônoma.

Um campo vetorial planar $X$ pode ser representado por meio do seguinte operador diferencial

$$
X=X_{1} \frac{\partial}{\partial x_{1}}+X_{2} \frac{\partial}{\partial x_{2}},
$$

definido no conjunto das funções de classe $C^{r}, r \geq 1$, isto é, dada $f \in C^{r}, r \geq 1$, temos

$$
X f=X_{1} \frac{\partial f}{\partial x_{1}}+X_{2} \frac{\partial f}{\partial x_{2}}
$$

representando a derivada de $f \circ \varphi$, para qualquer solução $\varphi$ de $(1.1 .1)$, com $\varphi(t)=x=$ $\left(x_{1}, x_{2}\right)$.

Definição 1.1.2. Seja $X: \Delta \subset \mathbb{R}^{2} \rightarrow \mathbb{R}^{2}$ um campo vetorial no plano. Considere o conjunto $\Omega=\{(t, x) \in \mathbb{R} \times \Delta ; t \in I\}$. Chamamos de fluxo de $X$ à aplicação $\varphi: \Omega \rightarrow \Delta$ definida por $\varphi(t, x)=\varphi_{x}(t)$, onde $I$ indica o intervalo maximal não degenerado para $o$ qual $\varphi_{x}(t)$ é curva integral de $X$.

Se $\Omega=\mathbb{R} \times \Delta$ então o fluxo de $X$ é dito fluxo global.

Em [49], encontramos a prova das propriedades abaixo listadas. As duas primeiras nos garantem que as aplicações $\varphi_{t}$, fluxos de um campo vetorial $X$ definem uma estrutura de grupo a um parâmetro de transformações:

- $\varphi(t+s, x)=\varphi(t, \varphi(s, x))$;

- $\varphi(-t, x)=(\varphi(t, x))^{-1}$.

Do Teorema de Existência e Unicidade das Equações Diferenciais Ordinárias segue que, para cada $x \in \Delta$ existe um aberto $I_{x}$ no qual a solução maximal $\varphi_{x}$, do campo $X$, é única e está definida, além disso, $\varphi_{x}(0)=x$.

Finalmente, a continuidade do fluxo com respeito às condições iniciais. Seja $\Omega=$ $\left\{(t, x): x \in \Delta\right.$ e $\left.t \in I_{x}\right\}$. O conjunto $\Omega$ é um aberto de $\mathbb{R}^{3}$ e o fluxo $\varphi: \Omega \rightarrow \mathbb{R}^{2}$, do campo $X$ de classe $C^{r}$, dado por $\varphi(t, x)=\varphi_{x}(t)$ é de classe $C^{r}$. 
Definição 1.1.3. A imagem da curva integral de $X$ pelo ponto $P$, isto é, o conjunto $\gamma_{P}=\left\{\varphi(t, P), t \in I_{P}\right\}$, é chamada de órbita de $X$ por $P$.

Definição 1.1.4. O conjunto aberto $\Delta$, domínio do campo $X$, é denominado de espaço de fase de X. O espaço de fase de X, munido da decomposição em órbitas do campo, é chamado de retrato de fase de $X$.

Definição 1.1.5. Um campo vetorial $X$ da forma $X: \mathbb{R}^{2} \rightarrow \mathbb{R}^{2}$ é linear se for da forma:

$$
X(x, y)=(a x+b y, c x+d y)
$$

onde $a, b, c, d \in \mathbb{R}$.

Definição 1.1.6. Seja o sistema de equações diferenciais no plano de classe $C^{r}$, com $r \geq 1$ ou $r=\omega$ (analítico), onde $P, Q \in C^{r}$

$$
\left\{\begin{array}{l}
\dot{x}=P(x, y) \\
\dot{y}=Q(x, y)
\end{array}\right.
$$

E seja

$$
X=P(x, y) \frac{\partial}{\partial x}+Q(x, y) \frac{\partial}{\partial x}
$$

o campo vetorial associado a (1.1.2), com p ponto singular de X. Dizemos que

$$
D X(0,0)=\left(\begin{array}{cc}
\frac{\partial P}{\partial x}(p) & \frac{\partial P}{\partial y}(p) \\
\frac{\partial Q}{\partial x}(p) & \frac{\partial Q}{\partial y}(p)
\end{array}\right)
$$

é a parte linear do campo vetorial $X$ em $p$.

Definição 1.1.7. Uma órbita $\gamma$ de um campo vetorial $X$ é dita periódica se existe $\tau>0$ tal que $\gamma(t+\tau)=\gamma(t), \forall t \in \mathbb{R}$. Se $\tau$ for tal que $\gamma\left(t_{1}\right) \neq \gamma\left(t_{2}\right)$, para $\left|t_{1}-t_{2}\right|<\tau$, então $\tau$ é chamado de período de $\gamma$. 
Definição 1.1.8. Uma órbita periódica $\gamma$ de um campo vetorial $X$ é chamada de ciclo limite se existe uma vizinhança $V$ de $\gamma$, tal que $\gamma$ seja isolada, isto é, é a única órbita fechada de $X$ que intercepta $V$.

Definição 1.1.9. Seja $x \in \Delta$. Dizemos que $x$ é um ponto:

- regular, se $X(x) \neq 0$;

- singular, ou uma singularidade, se $X(x)=0$.

Definição 1.1.10. Seja p um ponto singular de um campo vetorial da forma (1.1.3), de classe $C^{r}$, com $r \geq 1$ ou $r=\omega$ (analítico). O ponto singular $p$ é denominado:

(a) não degenerado, se 0 não é um autovalor de $D X(p)$;

(b) degenerado, se $D X(p)$ é degenerada, isto é, seu determinante é nulo. Um ponto singular degenerado é chamado de:

(b.1) degenerado elementar, se apenas um dos autovalores de $D X(p)$ for nulo;

(b.2) nilpotente, se os dois autovalores de $D X(p)$ são nulos, mas $D X(p) \neq 0$;

(b.3) linearmente nulo, se $D X(p)$ for identicamente nula;

(c) centro, se existe uma vizinhança aberta $V$ de $p$ tal que, todas as órbitas de $V \backslash\{p\}$ são órbitas periódicas. A singularidade é dita centro linear se ambos os autovalores de $D X(p)$ são números imaginários puros não nulos.

(d) foco, se existe uma vizinhança aberta $V$ de $p$ tal que, todas as órbitas de $V \backslash\{p\}$ espiralam para $p$ quando $t \rightarrow+\infty$ ou $t \rightarrow-\infty$.

Definição 1.1.11. Seja $p$ um ponto singular de um campo vetorial da forma (1.1.3), de classe $C^{r}$, com $r \geq 1$ ou $r=\omega$ (analítico). O ponto singular $p$ é dito monodrômico se não existem órbitas tendendo a, ou deixando $p$ com um ângulo bem definido.

Quando o campo vetorial $X$ é analítico, um ponto monodrômico é sempre um centro ou um foco. Este fato foi demonstrado no início da década de noventa do século passado, 
e é consequência dos Teoremas de Finitude dos Ciclos Limites de Ecalle e Ilyashenko - cf. [33].

Se o campo vetorial não for analítico, podem existir pontos monodrômicos que são pontos de acumulação de ciclos limites, como ilustra o exemplo a seguir.

Exemplo 1.1.12. [44]

Seja o sistema diferencial dado por:

$$
\left\{\begin{array}{l}
\left\{\begin{array}{l}
\dot{x}=-y+x F(x, y), \\
\dot{y}=x+y F(x, y), \quad \text { se }(x, y) \neq(0,0)
\end{array}\right. \\
(0,0), \text { caso contrário. }
\end{array}\right.
$$

onde $F(x, y)=\sqrt{x^{2}+y^{2}} \operatorname{sen}\left(\frac{1}{x^{2}+y^{2}}\right)$.

Portanto, a origem é um ponto singular do sistema.

Em coordenadas polares, temos

$$
\left\{\begin{array}{l}
\dot{r}=r^{2} \operatorname{sen}\left(\frac{1}{r}\right), \\
\dot{\theta}=1,
\end{array}\right.
$$

para $r>0$, com $\dot{r}=0$ em $r=0$.

Logo os círculos $\Gamma(n)=\left\{r=\frac{1}{n \pi}, n \in \mathbb{N}^{*}\right\}$ são trajetórias do sistema, uma vez que temos $\dot{r}=0$ ao longo deles.

Além disso, para $n \pi<\frac{1}{r}<(n+1) \pi$, segue que $\dot{r}<0$, se $n$ é ímpar e $\dot{r}>0$, se $n$ é par. Isto é, as trajetórias entre os círculos $\Gamma(n)$ espiralam para dentro ou para fora de um destes círculos.

Assim, $\Gamma(n)$ são ciclos limites que se acumulam na origem.

Definição 1.1.13. Um ponto singular $p$ de um campo vetorial de classe $C^{r}$, com $r \geq 1$ ou $r=\omega$ (analítico) é chamado de foco fraco quando p é um foco cuja parte linear é um centro, isto é, se for um centro linear que efetivamente é um foco do sistema. 
Nas definições que seguem abaixo $X$ indica um campo de vetores de classe $C^{r}$, com $r \geq 1$ ou $r=\omega$ (analítico), definido no aberto $\Delta \subseteq \mathbb{R}^{2}$.

Definição 1.1.14. Uma aplicação $f: A \rightarrow \Delta$ de classe $C^{r}$, é chamada de seção transversal local de $X$ quando, para cada $a \in A, f^{\prime}(a)$ e $X(f(a))$ são linearmente independentes. Considere $\Sigma=f(A)$ com a topologia induzida. Se $f: A \rightarrow \Sigma$ é um homeomorfismo, então $\Sigma$ é uma seção transversal de $X$.

O conceito de seção transversal acima foi enunciado apenas para o caso $n=2$. Em [49] encontramos definição semelhante para subconjuntos abertos de $\mathbb{R}^{n}, n \geq 2$, quando o espaço transversal é homeomorfo a $\mathbb{R}^{n-1}$.

\subsection{Classificação local de campos de vetores}

Nessa seção trataremos de definir os tipos de equivalência entre campos vetoriais planares e investigar suas consequências.

Definição 1.2.1. Sejam $X_{1}$ e $X_{2}$ dois campos vetoriais definidos em subconjuntos abertos $\Delta_{1}$ e $\Delta_{2}$ de $\mathbb{R}^{2}$, respectivamente. Dizemos que $X_{1}$ é topologicamente equivalente (respectivamente $C^{r}$-equivalente, com $r \geq 1$ ou $\left.r=\omega\right)$ a $X_{2}$ se existe um homeomorfismo (respectivamente um difeomorfismo de classe $C^{r}$ ) $h: \Delta_{1} \rightarrow \Delta_{2}$ que leva órbitas de $X_{1}$ em órbitas de $X_{2}$ preservando a orientação. Mais precisamente, sejam $p \in X_{1}$ e $\gamma_{p}^{1}$ órbita orientada de $X_{1}$ passando pelo ponto $p$. Então, $h\left(\gamma_{p}^{1}\right)$ é órbita orientada de $X_{2}$ passando pelo ponto $h(p)$.

O homeomorfismo $h$, da definição acima, é chamado de equivalência topológica (respectivamente $C^{r}$-equivalência) entre $X_{1}$ e $X_{2}$.

Definição 1.2.2. Sejam $\varphi_{1}: \Omega_{1} \rightarrow \mathbb{R}^{2}$ e $\varphi_{2}: \Omega_{2} \rightarrow \mathbb{R}^{2}$ os fluxos gerados pelos campos de vetores $X_{1}: \Delta_{1} \rightarrow \mathbb{R}^{2}$ e $X_{2}: \Delta_{2} \rightarrow \mathbb{R}^{2}$, respectivamente. Dizemos que $X_{1}$ é topologicamente conjugado (respectivamente $C^{r}$-conjugado) a $X_{2}$ quando existe um homeomorfismo (respectivamente um difeomorfismo de classe $\left.C^{r}\right) h: \Delta_{1} \rightarrow \Delta_{2}$ tal que $h\left(\varphi_{1}(t, x)\right)=$ $\varphi_{2}(t, h(x))$ para todo $(t, x) \in \Omega_{1}$. 
Na definição acima os intervalos maximais $I_{x}$, para $\varphi_{1}$, e $I_{h(x)}$ para $\varphi_{2}$ coincidem. O homeomorfismo (ou difeomorfismo) $h$ é chamado de uma conjugação topológica (respectivamente uma $C^{r}$-conjugação) entre os campos $X_{1}$ e $X_{2}$.

Teorema 1.2.3 (Teorema do Fluxo Tubular). Seja $p$ um ponto regular de um campo de vetores $X: \Delta \rightarrow \mathbb{R}^{2}$ de classe $C^{r}$, com $r \geq 1$ ou $r=\omega$ (analítico), e seja $f: A \rightarrow \Sigma$ uma seção transversal de $X$, de classe $C^{r}$, com $f(0)=p$. Então existe uma vizinhança do ponto p em $V \subset \Delta$ e um difeomorfismo $h: V \rightarrow(-\varepsilon, \varepsilon) \times B$ de classe $C^{r}$, para $\varepsilon>0$ e $B$ é um intervalo aberto com centro na origem, tal que:

1. $h(\Sigma \cap V)=0 \times B$;

2. $h$ é uma conjugação de classe $C^{r}$ entre $\left.X\right|_{V}$ e o campo de vetores constante $Y$ : $(-\varepsilon, \varepsilon) \times B \rightarrow \mathbb{R}^{2}$ definido por $Y(x, y)=(0,1)$.

Corolário 1.2.4. Seja $\Sigma$ uma seção transversal de um campo vetorial $X$ de classe $C^{r}$, com $r \geq 1$ ou $r=\omega$ (analítico). Para todo ponto $p \in \Sigma$, existem $\varepsilon=\varepsilon(p)>0$, uma vizinhança $V$ de $p$ em $\mathbb{R}^{2}$ e uma função $\tau: V \rightarrow \mathbb{R}$ de classe $C^{r}$, tal que $\tau(V \cap \Sigma)=0$ e:

(i) para todo $q \in V$, a curva integral $\varphi(t, q)$ de $\left.X\right|_{V}$ é definida e biunívoca em $J_{q}=$ $(-\varepsilon+\tau(q), \varepsilon+\tau(q)$

(ii) $\xi(q)=\varphi(\tau(q), q) \in \Sigma$ é o único ponto onde $\left.\varphi(\cdot, q)\right|_{J_{q}}$ intercepta $\Sigma$. Em particular, $q \in \Sigma \cap V$ se, e somente se, $\tau(q)=0$;

(iii) $\xi: V \rightarrow \Sigma e ́$ de classe $C^{r}$ e $D \xi(q)$ é sobrejetora para todo $q \in V$. Mais ainda, $D \xi(q) v=0$ se, e somente se, $v=\alpha X(q)$ para algum $\alpha \in \mathbb{R}$.

O teorema do fluxo tubular garante a existência de um difeomorfismo (de classe $C^{r}$ ) que conjuga um campo $X$ em uma vizinhança de um ponto regular qualquer $\left(x_{0}, y_{0}\right)$ ao campo constante $Y=(1,0)$. Deste modo, temos um conhecimento satisfatório das órbitas do campo vetorial $X$ nas vizinhanças de seus pontos regulares.

Entretanto, se $\left(x_{0}, y_{0}\right)$ é uma singularidade, a situação é bem mais complexa. A seguir, apresentamos o teorema de Hartman Grobman, que nos permite caracterizar o comportamento de um campo de vetores numa vizinhança de singularidades hiperbólicas. 
Definição 1.2.5. Um ponto singular $p$ de um campo de vetores $X=\left(f_{1}, \ldots, f_{m}\right)$, de classe $C^{1}$, é chamado de ponto hiperbólico se os autovalores de:

$$
D X(p)=\left[\begin{array}{ccc}
\frac{\partial f_{1}}{\partial x_{1}}(p) & \cdots & \frac{\partial f_{1}}{\partial x_{m}}(p) \\
\vdots & \ddots & \vdots \\
\frac{\partial f_{m}}{\partial x_{1}}(p) & \cdots & \frac{\partial f_{m}}{\partial x_{m}}(p)
\end{array}\right]
$$

têm parte real diferente de zero.

Os nós, as selas e os focos são hiperbólicos. Já o centro nos fornece um exemplo de singularidade não hiperbólica.

Teorema 1.2.6 (Teorema de Hartman-Grobman). Sejam $\Delta \subseteq \mathbb{R}^{m}$ aberto, $X: \Delta \rightarrow \mathbb{R}^{m}$ um campo de vetores de classe $C^{1}$ e $0 \in \Delta$ uma singularidade hiperbólica. Então $X$ e $D X(0)$ são localmente topologicamente equivalentes na origem.

Como se trata de um problema local, se a singularidade $\left(x_{0}, y_{0}\right) \in \Delta$ não estiver na origem, então por meio de uma carta local conveniente é possível levar esta singularidade à origem, preservando o comportamento do campo nas vizinhanças da singularidade.

O Teorema de Hartman-Grobman nos diz que, na vizinhança de uma singularidade hiperbólica, o comportamento de um campo não linear é o mesmo da sua parte linear.

Apresentamos abaixo, uma interpretação geométrica do teorema de Hartman-Grobman, para o caso geral em que a singularidade $\left(x_{0}, y_{0}\right)$ não está na origem:
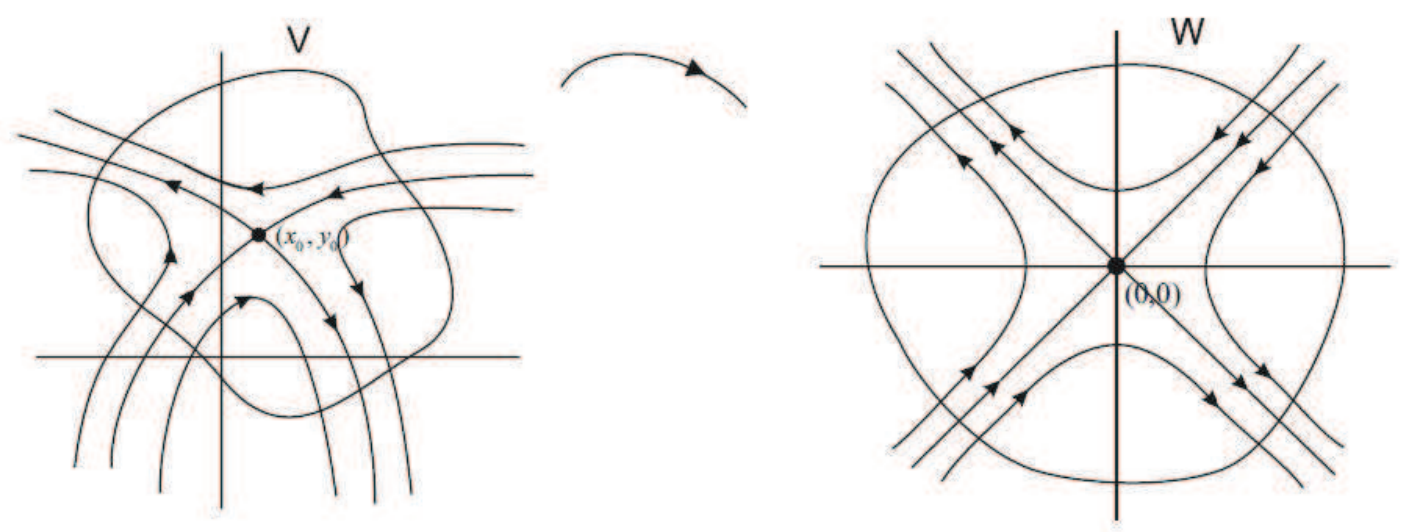

Figura 1.1: Interpretação geométrica do teorema de Hartman-Grobman 
A demonstração deste teorema pode ser encontrada em [43].

O próximo lema nos garante que dado um sistema diferencial analítico com uma singularidade isolada, podemos assumir, sem perda de generalidade, que este ponto singular é a origem.

Lema 1.2.7. Se $\left(x_{0}, y_{0}\right)$ é um ponto singular do sistema (1.1.2), então a origem é ponto singular do sistema

$$
\left\{\begin{aligned}
\dot{\bar{x}} & =P(\bar{x}, \bar{y}), \\
\dot{\bar{y}} & =Q(\bar{x}, \bar{y})
\end{aligned}\right.
$$

onde $x=\bar{x}+x_{0}, y=\bar{y}+y_{0}$, com $P(\bar{x}, \bar{y}), Q(\bar{x}, \bar{y})$ sem termos constantes.

A demonstração é trivial, bastando efetuar a mudança de coordenadas acima e voltar à notação original em $x$ e $y$.

Considerando a origem como uma singularidade isolada do campo vetorial planar $X$ e utilizando a equivalência acima definida, vamos investigar o comportamento local do campo $X$ numa vizinhança da origem. Esse estudo depende da classificação do ponto singular.

Proposição 1.2.8. [4] Seja X uma campo vetorial planar analítico, escrito na forma (1.1.2), com ponto singular não degenerado e isolado na origem. Suponha que os autovalores de sua parte linear na origem são números complexos conjugados $\lambda_{1,2}=\alpha \pm i \beta, \alpha, \beta \in$ $\mathbb{R}, \beta \neq 0$. Então, este sistema pode ser escrito na forma

$$
\left\{\begin{array}{l}
\dot{x}=\alpha x-\beta y+F(x, y), \\
\dot{y}=\beta x+\alpha y+G(x, y)
\end{array}\right.
$$

onde $F(x, y)$ e $G(x, y)$ são funções analíticas numa vizinhança da origem, com $F(0,0)=$ $G(0,0)=D F(0,0)=D G(0,0)=0$.

Demonstração. Da analiticidade do campo $X$ numa vizinhança da origem, podemos expressar $P(x, y)$ e $Q(x, y)$ como:

$$
P(x, y)=P_{x}^{\prime}(0,0) x+P_{y}^{\prime}(0,0) y+\Phi(x, y)
$$




$$
Q(x, y)=Q_{x}^{\prime}(0,0) x+Q_{y}^{\prime}(0,0) y+\Psi(x, y)
$$

onde as funções $\Phi(x, y)$ e $\Psi(x, y)$ são analíticas e $\Phi(0,0)=\Psi(0,0)=0, \Phi_{x}^{\prime}(0,0)=$ $\Phi_{y}^{\prime}(0,0)=\Psi_{x}^{\prime}(0,0)=\Psi_{y}^{\prime}(0,0)=0$.

Denotando $P_{x}^{\prime}(0,0), P_{y}^{\prime}(0,0), Q_{x}^{\prime}(0,0), Q_{y}^{\prime}(0,0)$ respectivamente por $a, b, c, d$, segue que o sistema (1.1.2) passa a ser escrito na forma

$$
\left\{\begin{array}{l}
\dot{x}=a x+b y+\Phi(x, y), \\
\dot{y}=c x+d y+\Psi(x, y) .
\end{array}\right.
$$

Portanto, a parte linear $D X(0,0)$ do sistema (1.2.2) tem seu polinômio característico dado por $p(\lambda)=\lambda^{2}-(a+d) \lambda+(a d-b c)$.

Como a origem é um ponto singular não degenerado e isolado, segue que $a d-b c \neq 0$. Assim, $\triangle=(a+d)^{2}-4(a d-b c)=(a-d)^{2}-4 a c$.

Por outro lado, como $\lambda_{1,2}=\alpha \pm i \beta, \alpha, \beta \in \mathbb{R}, \beta \neq 0$, vale:

$$
\triangle=(a-d)^{2}-4 a c<0 \Longrightarrow a, c \neq 0
$$

Considere a mudança de coordenadas:

$$
\left\{\begin{array}{l}
x=-\left(\frac{\alpha-d}{\beta c}\right) \bar{x}-\frac{1}{c} \bar{y} \\
y=-\frac{1}{\beta} \bar{x}
\end{array}\right.
$$

A condição (1.2.3) e o fato de que $\beta \neq 0$ asseguram que esta mudança de coordenadas é sempre possível.

Assim, a expressão (1.2.2), após a mudança de coordenadas (1.2.4), será:

$$
\left\{\begin{array}{l}
\dot{\bar{x}}=\alpha \bar{x}-\beta \bar{y}+\bar{F}(\bar{x}, \bar{y}), \\
\dot{\bar{y}}=\beta \bar{x}+\alpha \bar{y}+\bar{G}(\bar{x}, \bar{y}),
\end{array}\right.
$$

onde $\bar{F}(\bar{x}, \bar{y}), \bar{G}(\bar{x}, \bar{y})$ são analíticas, com $\bar{F}(0,0)=\bar{G}(0,0)=0$, e $\bar{F}_{x}^{\prime}(0,0)=\bar{F}_{y}^{\prime}(0,0)=$ $\bar{G}_{x}^{\prime}(0,0)=\bar{G}_{y}^{\prime}(0,0)=0$. 
Retornando à notação inicial nas variáveis $x, y$, obtemos a forma desejada (1.2.1).

Para o caso particular em que os autovalores da parte linear $D X(0,0)$ do sistema (1.2.2) são números imaginários puros não nulos, isto é, da forma $\lambda_{1,2}= \pm \beta, \beta \neq 0$, obtemos, a partir de (1.2.1):

$$
\left\{\begin{array}{l}
\dot{\bar{x}}=-\beta \bar{y}+\bar{F}(\bar{x}, \bar{y}), \\
\dot{\bar{y}}=\beta \bar{x}+\bar{G}(\bar{x}, \bar{y}),
\end{array}\right.
$$

onde $\bar{F}(\bar{x}, \bar{y}), \bar{G}(\bar{x}, \bar{y})$ analíticas, com $\bar{F}(0,0)=\bar{G}(0,0)=0$, e $\bar{F}_{x}^{\prime}(0,0)=\bar{F}_{y}^{\prime}(0,0)=$ $\bar{G}_{x}^{\prime}(0,0)=\bar{G}_{y}^{\prime}(0,0)=0$.

Como $\beta \neq 0$, podemos efetuar um reescalonamento do tempo $\beta t=\tau$. Retornando à notação inicial nas variáveis $x, y, t$, obtemos:

$$
\left\{\begin{array}{l}
\dot{x}=-y+F(x, y) \\
\dot{y}=x+G(x, y)
\end{array}\right.
$$

Observe que o reescalonamento do tempo preserva a analiticidade do sistema e portanto, $F(x, y), G(x, y)$ são analíticas e satisfazem $F(0,0)=G(0,0)=D F(0,0)=D G(0,0)=$ 0.

Proposição 1.2.9. Se p é uma singularidade degenerada, então $D X(p)$ tem pelo menos um autovalor nulo.

Demonstração. Sejam $m$ o traço de $D X(p)$ e $n$ o valor de seu determinante. Então, o polinômio característico de $D X(p)$ é dado por $p(\lambda)=\lambda^{2}-m \lambda+n=\lambda^{2}-m \lambda$ pois, por hipótese, $n=0$. Logo, $p(\lambda)=0 \Leftrightarrow \lambda^{2}-m \lambda=0 \Leftrightarrow \lambda=0$ ou $\lambda=m$.

Proposição 1.2.10. [4] Seja um sistema diferencial analítico no plano da forma (1.1.2), tal que a origem seja um ponto singular isolado degenerado nilpotente. Então, este sistema pode ser escrito na forma

$$
\left\{\begin{array}{l}
\dot{x}=y+F(x, y) \\
\dot{y}=G(x, y)
\end{array}\right.
$$

onde $F(x, y)$ e $G(x, y)$ são funções analíticas numa vizinhança da origem, com $F(0,0)=$ $G(0,0)=D F(0,0)=D G(0,0)=0$. 
Demonstração. Repetindo parte dos procedimentos da demonstração da Proposição 1.2.8, segue que numa vizinhança da origem podemos expressar o sistema (1.1.2) na forma (1.2.2) e a parte linear $D X(0,0)$ do sistema (1.2.2) é dada por

$$
D X(0,0)=\left(\begin{array}{ll}
a & b \\
c & d
\end{array}\right)
$$

Como a origem é um ponto singular degenerado nilpotente, temos

$$
\left\{\begin{array}{l}
|a|+|b|+|c|+|d| \neq 0 \\
a+d=0 \\
a d-b c=0
\end{array}\right.
$$

Assim, $D X(0,0)$ é uma matriz nilpotente de ordem 2. De fato, aplicando as condições acima, temos

$$
\begin{aligned}
D X^{2}(0,0) & =\left(\begin{array}{ll}
a & b \\
c & d
\end{array}\right)^{2}=\left(\begin{array}{ll}
a^{2}+b c & a b+b d \\
a c+c d & b c+d^{2}
\end{array}\right)= \\
& =\left(\begin{array}{ll}
a(a+d) & b(a+d) \\
c(a+d) & d(a+d)
\end{array}\right)=\left(\begin{array}{ll}
0 & 0 \\
0 & 0
\end{array}\right)
\end{aligned}
$$

A Forma Canônica de Jordan para matrizes $2 \times 2$ nilpotentes (vide [31]) tem expressão

$$
\left(\begin{array}{ll}
0 & 0 \\
1 & 0
\end{array}\right)
$$

Agora, renomeando as coordenadas $x$ e $y$, trocando-as entre si, obtemos a expressão (1.2.6) desejada.

Proposição 1.2.11. [4] Seja um sistema diferencial analítico no plano da forma (1.1.2), tal que a origem seja um ponto singular degenerado linearmente nulo, com $D X(0,0)$ a matriz nula. Então, este sistema pode ser escrito na forma

$$
\left\{\begin{array}{l}
\dot{x}=F(x, y), \\
\dot{y}=G(x, y)
\end{array}\right.
$$

onde $F(x, y)$ e $G(x, y)$ são funções analíticas numa vizinhança da origem, com $F(0,0)=$ 
$G(0,0)=D F(0,0)=D G(0,0)=0$.

Demonstração. Pela hipótese de analiticidade do sistema (1.1.2), numa vizinhança da origem podemos expressar o sistema (1.1.2) na forma (1.2.2). Como por hipótese a parte linear $D X(0,0)$ é a matriz nula, o sistema tem a forma (1.2.7).

Em resumo, das três proposições acima segue que, se um sistema diferencial analítico planar da forma (1.1.2) tem uma singularidade isolada na origem, então após uma mudança de coordenadas analítica e um reescalonamento de tempo convenientes, este sistema pode ser escrito em uma das três formas seguintes:

$$
\left\{\begin{array}{l}
\dot{x}=-y+F(x, y) \\
\dot{y}=x+G(x, y)
\end{array}\right.
$$

se a origem for uma singularidade não degenerada, com $D X(0,0)$ possuindo autovalores puramente imaginários (centro linear);

$$
\left\{\begin{array}{l}
\dot{x}=y+F(x, y), \\
\dot{y}=G(x, y),
\end{array}\right.
$$

se a origem for uma singularidade degenerada nilpotente;

$$
\left\{\begin{array}{l}
\dot{x}=F(x, y), \\
\dot{y}=G(x, y),
\end{array}\right.
$$

se a origem for uma singularidade degenerada, com parte linear identicamente nula.

As funções $F(x, y)$ e $G(x, y)$ em cada caso são analíticas sem termos constantes ou lineares, definidas numa vizinhança da origem.

\subsection{Formas normais}

O principal resultado apresentado nesta seção é o Teorema Formal das Formas Normais, que fornece um método para obtenção de um sistema de coordenadas no qual o campo vetorial assume sua forma "mais simples possível", no sentido dado a seguir. A 
teoria das formas normais será fundamental no desenvolvimento de alguns resultados centrais desta dissertação, discutidos na Seção 3.1. Vale destacar que, a literatura sobre formas normais é vasta, e não a abordaremos em detalhes. O leitor interessado pode consultar $[17,52,30,47]$.

Em nosso contexto, "mais simples possível" significa remover termos não essenciais da série de Taylor das funções que definem o campo vetorial. Em um campo vetorial que possui apenas parte linear, isso significa escrevê-la na forma canônica de Jordan. Dado um ponto singular hiperbólico - cf. Definição 1.2.5, de um campo vetorial $X$, podemos utilizar o teorema de Hartman-Grobman para exibir uma equivalência local entre $X$ e sua parte linear. Entretanto, para singularidades não hiperbólicas, nem sempre isso pode ser feito e encontrar a forma "mais simples possível" do campo torna-se tarefa bem mais difícil.

Alguns aspectos importantes acerca deste método devem ser observados:

- o teorema formal das formas normais será apresentado para campos vetoriais de classe $C^{\infty}$ em $\mathbb{R}^{n}$, uma vez que a restrição a campos vetoriais no plano não resulta em maior simplicidade na demonstração;

- o método é local;

- a mudança de coordenadas é de fato uma composição de mudanças de coordenadas polinomiais locais, que mantém a parte linear do campo e remove termos de ordem superior da série de Taylor das funções que expressam o campo;

- a configuração da forma normal é determinada exclusivamente pela natureza da parte linear do campo vetorial.

Seja $X=A+f$ um campo vetorial em $\mathbb{R}^{n}$, com $A$ linear e $f$ uma função de classe $C^{\infty}$ tal que $f(0)=0$ e $D f(0)=0$. Queremos determinar, para cada campo vetorial linear $A$ dado, uma classe restrita de não linearidades $F_{n}$, tão pequenas e simples quanto for possível, de modo que para cada $f$, por uma mudança de coordenadas $C^{\infty}$ conveniente, possamos escrever o campo $X$ na forma $\hat{X}=A+\hat{f}, \hat{f} \in F_{n}$.

Antes de prosseguirmos, apresentamos algumas definições importantes.

Definição 1.3.1. [52] Sejam $\left\{s_{1}, s_{2}, \ldots, s_{n}\right\}$ uma base de $\mathbb{R}^{n}$, e y $=\left(y_{1}, y_{2}, \ldots, y_{n}\right)$ as coordenadas de um ponto $P \in \mathbb{R}^{n}$ qualquer, com respeito a esta base. 
Definimos um monômio vetorial de grau $k$ como $h_{k}=\left(y_{1}^{m_{1}}, y_{2}^{m_{2}}, \ldots, y_{n}^{m_{n}}\right) s_{i}$, com $\sum_{i=1}^{n} m_{i}=k, \quad m_{i} \in \mathbb{Z}_{+}$. O conjunto de todos os monômios vetoriais $h_{k}$ forma um espaço vetorial que denotaremos por $\mathcal{H}^{k}$. Naturalmente, este é o espaço vetorial dos polinômios homogêneos de grau $k$ em $\mathbb{R}^{n}$.

Uma base óbvia para $\mathcal{H}^{k}$ consiste nos elementos formados considerando-se todos os possiveis monômios de grau $k$ multiplicados por cada $s_{i}$.

Lembrando que, dados dois campos vetoriais $X, Y \in \mathcal{H}^{k}\left(\mathbb{R}^{n}\right)$, com $X=\sum_{i=1}^{n} a_{i} \frac{\partial}{\partial x_{i}}$, $Y=\sum_{i=1}^{n} b_{i} \frac{\partial}{\partial x_{i}}$, o colchete de Lie do $\operatorname{par}(X, Y)$ é o campo $[X, Y] \in \mathcal{H}^{k}\left(\mathbb{R}^{n}\right)$, dado por:

$$
[X, Y]=\sum_{i=1}^{n} c_{i} \frac{\partial}{\partial x_{i}}, \text { onde } c_{i}=\sum_{j=1}^{n} a_{j} \frac{\partial b_{i}}{\partial x_{j}}-b_{j} \frac{\partial a_{i}}{\partial x_{j}}
$$

Definição 1.3.2. No espaço vetorial $\mathcal{H}^{k}$, definimos o operador linear:

$$
\begin{aligned}
\operatorname{ad}_{k} A: \mathcal{H}^{k} & \longrightarrow \mathcal{H}^{k} \\
X & \mapsto[A, X],
\end{aligned}
$$

onde [.,.] denota o colchete de Lie.

Na literatura dedicada ao tema, este operador muitas vezes é denominado de "operador homológico- cf. [47].

Para uma demonstração da linearidade do operador $a d_{k} A$ em $\mathcal{H}^{k}$, consulte [52].

Seja $B^{k}$ a imagem do operador $a d_{k} A$ em $\mathcal{H}^{k}$, e seja $G^{k}$ o espaço complementar a $B^{k}$ em $\mathcal{H}^{k}$. Então:

$$
\mathcal{H}^{k}=B^{k} \oplus G^{k}
$$

Com base nessas informações, enunciamos o teorema abaixo:

Teorema 1.3.3 (Teorema Formal das Formas Normais). [22] Seja $X$ um campo vetorial de classe $C^{r}, r \geq 1$, definido numa vizinhança de zero, com $X(0)=0$ e $D X(0)=$ 
A. Sejam $B^{k}=a d_{k} A\left(\mathcal{H}^{k}\right)$ e $G^{k}$ seu espaço complementar, isto é, $\mathcal{H}^{k}=B^{k} \oplus G^{k}$. Então, existe uma mudança de coordenadas analítica, numa vizinhança da origem, que transforma $\dot{x}=X(x)$ em $\dot{y}=A y+g_{2}(y)+g_{3}(y)+\ldots+g_{r}(y)+o\left(\left\|y^{r}\right\|\right)$, onde $g_{i} \in G^{i}, i=$ $1,2, \ldots, r$.

A demonstração completa deste teorema pode ser encontrada em [17, 30]. A seguir, apresentamos um esboço da mesma.

Esboço da demonstração. A prova é feita por indução em $s, 2 \leq s \leq r$. Suponha que $\dot{x}=X(x)$ tenha sido transformada e que os termos de grau menor que $s$ estão em $G^{i}$ :

$$
\dot{x}=X(x)=A x+g_{2}(x)+g_{3}(x)+\ldots+g_{s-1}(x)+f_{s}(x)+o\left(\left\|x^{s}\right\|\right),
$$

onde $G^{i}$ é o espaço complementar de $B^{i}$ em $\mathcal{H}^{i}, i=1,2, \ldots, s-1$, e $f_{s} \in \mathcal{H}^{s}$. Fazendo a mudança de coordenadas $x=y+P(y)$, com $P \in \mathcal{H}^{s}$, temos:

$$
(I+D P(y)) \dot{y}=A(y+P(y))+g_{2}(y)+\ldots+g_{s-1}(y)+f_{s}(y)+o\left(\left\|y^{s}\right\|\right) .
$$

Usando o fato de que $\left(I+D P(y)^{-1}\right)=I-D P(y)+\tilde{o}\left(\left\|y^{s}\right\|\right)$ e efetuando os demais cálculos temos:

$$
\dot{y}=A y+g_{2}(y)+\ldots+g_{s-1}(y)+f_{s}(y)+A P(y)-D P(y) A y+\bar{o}\left(\left\|y^{s}\right\|\right) .
$$

Os termos de ordem menor que $s$ não são alterados pela mudança de coordenadas, enquanto que o termo de grau $s$ é dado por: $f_{s}(y)+A P(y)-D P(y) A y=f_{s}(y)+$ $a d_{k} A(P(y))$. Então, $P$ deve ser tal que $g_{s}(y)=f_{s}(y)-a d_{k} A(P(y)) \in G^{s}$.

\subsection{Desingularização de pontos singulares}

O Teorema de Hartman-Grobman - cf. Teorema 1.2.6, possibilita caracterizar localmente os campos vetoriais $X$ com singularidade hiperbólica isolada. Entretanto, para singularidades não hiperbólicas, este resultado não se aplica e, por isso, temos que recorrer a outros métodos. 
O método blow-up é usado para encontrar o retrato de fase de campos de vetores $X$ planares, numa vizinhança de uma singularidade não hiperbólica.

Essencialmente, podemos dizer que blow up é a técnica em que uma mudança de coordenadas conveniente "explode" uma singularidade em uma curva na qual obtemos, genericamente, finitas singularidades. Após nova mudança de coordenadas, estudamos as singularidades do campo resultante $\bar{X}$, isoladamente, ao longo desta curva. Para as singularidades não hiperbólicas, podemos aplicar novo blow up, e assim sucessivamente. Sob condições genéricas, após uma sequência finita de blow ups, são obtidas apenas singularidades elementares. Esse resultado é conhecido como Teorema da Decomposição. Pelo processo inverso (blow down), obtém-se o retrato de fase local do campo original $X$. Maiores detalhes acerca de blow up e do Teorema da Decomposição podem ser encontrados em [22].

Esta técnica também serve para estudar pontos fixos de difeomorfismos, mas vamos nos restringir à aplicação do blow-up na investigação de singularidades de campos de vetores.

\section{Germes e jatos de campos de vetores}

Definição 1.4.1. Dois campos de vetores $X$ e $Y$ em $\mathbb{R}^{n}$, com $X(0)=Y(0)=0$, são germe-equivalentes em 0 se coincidem em alguma vizinhança $V$ de 0 , isto é

$$
X(x)=Y(x), \forall x \in V .
$$

As classes de equivalência para esta relação de equivalência são chamadas germes de campos vetoriais em 0 . Denotamos por $G^{n, r}$ o conjunto de germes para campos de vetores de classe $C^{r}$ em 0 , e por $G^{n}$ o conjunto de germes para campos $C^{\infty}$ em 0 .

Definição 1.4.2. Sejam $\tilde{X}, \tilde{Y} \in G^{n}$. Então $\tilde{X}$ e $\tilde{Y}$ são $k$-jato-equivalentes $($ com $k \in \mathbb{N})$ se para algum representante $X$ e $Y$, respectivamente de $\tilde{X}$ e $\tilde{Y}$, tivermos

$$
\exists c>0, \exists \delta>0 ;\|(X-Y)(x)\| \leq c\|x\|^{k+1}, \forall x \in \mathbb{R}^{n},\|x\|<\delta .
$$

Uma classe de equivalência para esta relação é chamada k-jato de um campo de vetores 
e será denotada por $j_{k}(X)(0)$. A classe de equivalência $j_{k}(X)(0)$ é formada por germes cujo representante é $X$.

Quando $X$ é de classe $C^{2}$ e $X(0)=0$, pela diferenciabilidade obtemos $X(x)=$ $D X(0) x+r_{1}(x)$, com $\lim _{x \rightarrow 0} \frac{r_{1}}{\|x\|^{2}}=0$. Então, $\lim _{x \rightarrow 0}(X(x)-D X(0) x)=0$. Neste caso, $\tilde{X}$ e $\tilde{A}$ são 1-jato-equivalentes. Portanto, $j_{1}(X)(0)=j_{1}(D X(0))(0)$.

Por este motivo, denotamos $D X(0)$ por $j_{1}(D X(0))(0)$. Analogamente, dizemos que $j_{k}(X)(0)=0$ se 0 é um representante do jato. Isto é,

$$
\exists c>0, \exists \delta>0 ;\|(X)(x)\| \leq c\|x\|^{k+1}, \forall x \in \mathbb{R}^{n},\|x\|<\delta .
$$

\section{Blow up polar em $\mathbb{R}^{n}$}

Descreveremos o método para campos vetoriais $C^{\infty}$. Seja $X$ um campo vetorial planar de classe $C^{\infty}, \operatorname{com} X(0)=0$, definido por $X(x, y)=P(x, y) \frac{\partial}{\partial x}+Q(x, y) \frac{\partial}{\partial y}$, associado ao sistema:

$$
\left\{\begin{array}{l}
\dot{x}=P(x, y), \\
\dot{y}=Q(x, y),
\end{array}\right.
$$

Considere a aplicação $\phi$ correspondente à mudança de coordenadas dada por

$$
\begin{aligned}
\phi: \mathbb{S}^{1} \times \mathbb{R} & \rightarrow \mathbb{R}^{2} \\
(\theta, r) & \mapsto(r \cos (\theta), r \operatorname{sen}(\theta))
\end{aligned}
$$

Observe que $\phi$ leva $\{r=0\}$ em $(0,0)$ e a aplicação inversa $\phi^{-1}$ "expande"a origem em um círculo.

Queremos encontrar um campo de vetores $\hat{X}: \mathbb{S}^{1} \times \mathbb{R} \rightarrow \mathbb{R}^{2}$, com

$$
\hat{X}=X(\theta, r)=X_{1}(\theta, r) \frac{\partial}{\partial \theta}+X_{2}(\theta, r) \frac{\partial}{\partial r}
$$

de tal forma que o sistema

$$
\left\{\begin{array}{l}
\dot{\theta}=X_{1}(\theta, r), \\
\dot{r}=X_{2}(\theta, r),
\end{array}\right.
$$

seja equivalente ao sistema (1.4.1). 
O campo $\hat{X}$ é chamado "pull back" de $X$ por $\phi$. Pela Eq. $(1.4 .2), x=r \cos (\theta)$ e $y=r \operatorname{sen}(\theta)$, efetuando cálculos algébricos simples (cálculos similares serão demonstrados com mais detalhes na Seção 1.4 obtemos:

$$
\left\{\begin{aligned}
\dot{\theta} & =\frac{1}{r}(\cos (\theta) Q(r \cos (\theta), r \operatorname{sen}(\theta))-\operatorname{sen}(\theta) P(r \cos (\theta), r \operatorname{sen}(\theta))), \\
\dot{r} & =\cos (\theta) P(r \cos (\theta), r \operatorname{sen}(\theta))+\operatorname{sen}(\theta) Q(r \cos (\theta), r \operatorname{sen}(\theta)) .
\end{aligned}\right.
$$

Portanto,

$$
\begin{aligned}
\hat{X}(\theta, r)= & \frac{1}{r}[\cos (\theta) Q(r \cos (\theta), r \operatorname{sen}(\theta))-\operatorname{sen}(\theta) P(r \cos (\theta), r \operatorname{sen}(\theta))] \frac{\partial}{\partial \theta} \\
& +[\cos (\theta) P(r \cos (\theta), r \operatorname{sen}(\theta))+\operatorname{sen}(\theta) Q(r \cos (\theta), r \operatorname{sen}(\theta))] \frac{\partial}{\partial r}
\end{aligned}
$$

Tal construção nos permite demonstrar a seguinte proposição:

Proposição 1.4.3. Sejam $X$ um campo planar de classe $C^{\infty}$, tal que $X(0)=0$, e $\phi$ : $\mathbb{S}^{1} \times \mathbb{R}_{+}^{*} \rightarrow \mathbb{R}^{2} \backslash\{0\}$, definida em (1.4.2). Então existe um campo $\hat{X}: \mathbb{S}^{1} \times \mathbb{R}_{+}^{*} \rightarrow \mathbb{S}^{1} \times \mathbb{R}$, tal que $D \phi(\theta, r) \hat{X}(\theta, r)=X(\phi(\theta, r)), \forall(\theta, r) \in \mathbb{S}^{1} \times \mathbb{R}_{+}^{*}$.

Demonstração. Basta verificar que $\hat{X}$, dado por (1.4.4), satisfaz esta condição.

Da proposição anterior, segue que $\left.\phi\right|_{\mathbb{S}^{1} \times \mathbb{R}_{+}^{*}}$ é um difeomorfismo. Portanto, $\hat{X}$ é topologicamente conjugado a $\left.X\right|_{\mathbb{R}^{2} \backslash\{0\}}$ (vide Definição 1.2.2).

O campo $\hat{X}$ está definido na superfície do cilindro e, em $r=0$, a singularidade está "expandida", ou "desingularizada". A seguir, apresentamos um esboço do retrato de fase, no cilindro:

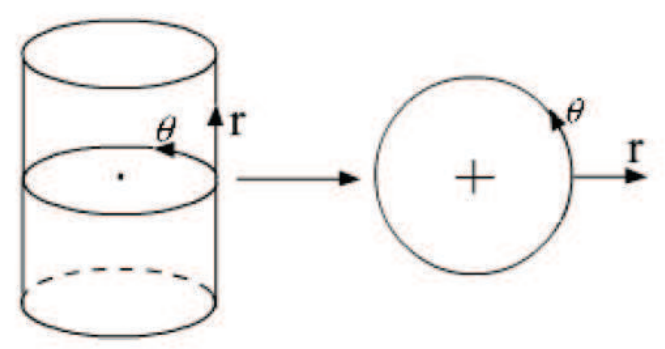

Figura 1.2: Esboço do retrato de fase do campo $\hat{X}$

Se $X$ tem a propriedade de $j_{k}(X)(0)=0$ e $j_{k+1}(X)(0) \neq 0$ para algum $k<\infty$, isto é, $\exists k \in \mathbb{Z}_{+}$, onde $k$ é o maior inteiro tal que $r^{k+1} \mid P(r \cos (\theta), r \operatorname{sen}(\theta))$ e $r^{k+1} \mid$ 
$Q(r \cos (\theta), r \operatorname{sen}(\theta))$, definimos $\bar{X}$ como:

$$
\bar{X}=\frac{1}{r^{k}} \hat{X}
$$

que é um campo de classe $C^{\infty}$ em $\mathbb{S}^{1} \times \mathbb{R}$.

Observe que a singularidade $(0,0)$ foi levada para a curva $\mathbb{S}^{1} \times\{0\}$. Além disso, ao longo de $\mathbb{S}^{1} \times\{0\}$, as singularidades são determinadas pelas soluções de $\bar{X}_{\theta}(\theta, 0)=0$.

Exemplo 1.4.4. Considere o campo $X=\left(x^{2}-2 x y, y^{2}-2 x y\right)$.

Fazendo a mudança de coordenadas $x=r \cos (\theta), y=r \operatorname{sen}(\theta)$ obtemos:

$$
\begin{aligned}
& P(r \cos (\theta), r \operatorname{sen}(\theta))=r^{2}\left(\cos ^{2}(\theta)-2 \cos (\theta) \operatorname{sen}(\theta)\right), \\
& Q(r \cos (\theta), r \operatorname{sen}(\theta))=r^{2}\left(\operatorname{se}^{2}(\theta)-2 \cos (\theta) \operatorname{sen}(\theta)\right),
\end{aligned}
$$

então,

$$
\begin{aligned}
\hat{X}(\theta, r)= & \left(r^{2}\left(\cos ^{3}(\theta)+\operatorname{sen}^{3}(\theta)-2 \cos ^{2}(\theta) \operatorname{sen}(\theta)+2 \operatorname{sen}^{2}(\theta) \cos (\theta)\right),\right. \\
& 3 r \cos (\theta) \operatorname{sen}(\theta)(\operatorname{sen}(\theta)-\cos (\theta)))
\end{aligned}
$$

Neste caso, $k=1$. Assim, por (1.4.5), temos:

$$
\begin{aligned}
\bar{X}(\theta, r)= & \left(r\left(\cos ^{3}(\theta)+\operatorname{sen}^{3}(\theta)-2 \cos ^{2}(\theta) \operatorname{sen}(\theta)+2 \operatorname{sen}^{2}(\theta) \cos (\theta)\right),\right. \\
& 3 \cos (\theta) \operatorname{sen}(\theta)(\operatorname{sen}(\theta)-\cos (\theta))) .
\end{aligned}
$$

Em $r=0$, temos $\theta=0, \frac{\pi}{2}, \frac{3 \pi}{2}, \pi, \frac{\pi}{4}, \frac{5 \pi}{4}$ como soluções de $\bar{X}_{\theta}(0, \theta)=0$. Agora, efetuamos estudo local do campo em cada ponto singular identificado.

$$
J \bar{X}(\theta, r)=\left(\begin{array}{cc}
\frac{\partial \bar{X}_{r}}{\partial r}(\theta, r) & \frac{\partial \bar{X}_{r}}{\partial \theta}(\theta, r) \\
\frac{\partial \bar{X}_{\theta}}{\partial r}(\theta, r) & \frac{\partial \bar{X}_{\theta}}{\partial \theta}(\theta, r)
\end{array}\right)
$$


Como $\frac{\partial \bar{X}_{\theta}}{\partial r}(\theta, r)=0$, segue que

$\operatorname{det} J \bar{X}(\theta, r)=\frac{\partial \bar{X}_{r}}{\partial r}(\theta, r) \frac{\partial \bar{X}_{\theta}}{\partial \theta}(\theta, r)=\left(\cos ^{3}(\theta)+\operatorname{sen}^{3}(\theta)-2 \cos ^{2}(\theta) \operatorname{sen}(\theta)+2 \operatorname{sen}^{2}(\theta) \cos (\theta)\right)$ $\left(6 \operatorname{sen}(\theta) \cos ^{2}(\theta)-3 \operatorname{sen}^{3}(\theta)+6 \cos (\theta) \operatorname{sen}^{2}(\theta)-3 \cos ^{3}(\theta)\right)$.

Então $\operatorname{det} J \bar{X}(0,0)=\operatorname{det} J \bar{X}(\pi, 0)=-3$. Logo, temos singularidades do tipo sela. Analogamente, efetuando os cálculos para $\theta=\frac{\pi}{2}, \frac{3 \pi}{2}, \frac{\pi}{4}, \frac{5 \pi}{4}$, temos pontos singulares do tipo sela. Aplicando um blow down, obtemos o seguinte retrato de fase de $X$ :

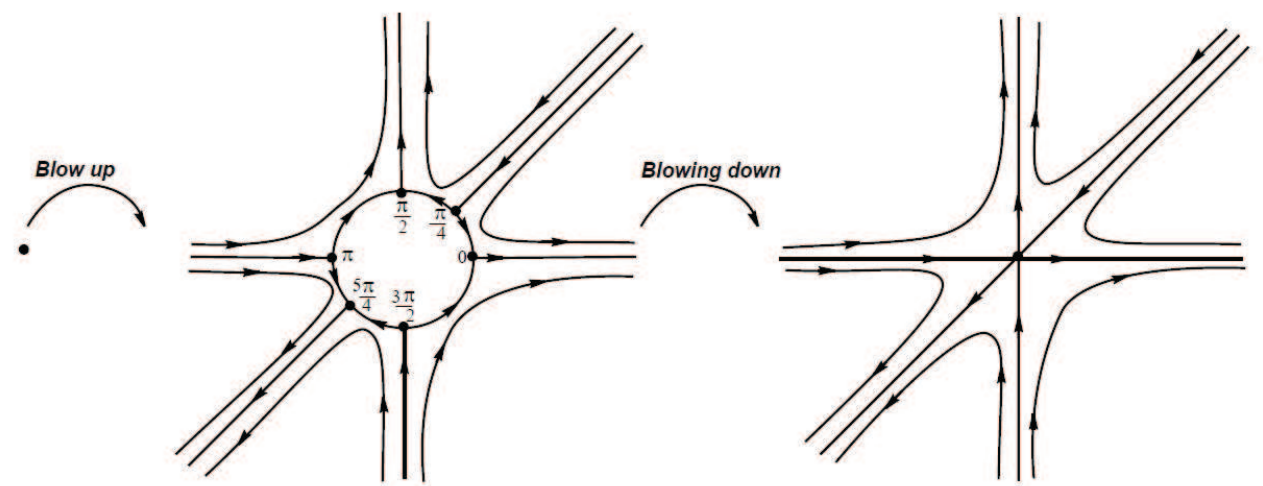

Figura 1.3: Exemplo de blow up polar

\section{Blow up direcional}

Blow up na direção x

Seja $X=(P(x, y), Q(x, y))$ um campo de vetores planar, com singularidade isolada na origem, e considere a mudança $x=u$ e $y=u v$. Então,

$$
\left\{\begin{array}{l}
\dot{x}=\dot{u} \\
\dot{y}=\dot{u} v+u \dot{v} .
\end{array}\right.
$$

e assim, temos:

$$
\tilde{X}(u, v)=\left\{\begin{array}{l}
\dot{u}=P(u, u v) \\
\dot{v}=\frac{Q(u, u v)-v P(u, u v)}{u}
\end{array}\right.
$$


Escreva $\tilde{X}(u, v)=\left(\tilde{X}_{u}, \tilde{X}_{v}\right)$, onde $\tilde{X}_{u}=P(u, u v)$ e $\tilde{X}_{v}=\frac{Q(u, u v)-v P(u, u v)}{u}$.

Então, seja $k$ o maior inteiro tal que $u^{k} \mid \tilde{X}_{u}$ e $u^{k} \mid \tilde{X}_{v}$.

Defina $\bar{X}(u, v)=\left(\bar{X}_{u}, \bar{X}_{v}\right)=\frac{\tilde{X}(u, u v)}{u^{k}}$. A singularidade $(0,0)$ é levada em $u=0$ e, para $u=0$ as singularidades de $\tilde{X}$ são determinadas pelas soluções de $\bar{X}_{v}=0$.

Blow up na direção y

Seja $X=(P(x, y), Q(x, y))$ um campo de vetores planar, com singularidade isolada na origem. Defina a mudança $x=u v$ e $y=v$. Então,

$$
\left\{\begin{array}{l}
\dot{x}=\dot{u} v+u \dot{v} \\
\dot{y}=\dot{u}
\end{array}\right.
$$

analogamente ao blow up direcional em $x$, definimos

$$
\tilde{X}(u, v)=\left(\tilde{X}_{u}, \tilde{X}_{v}\right)=\left(\frac{P(u v, v)-u Q(u v, v)}{v}, Q(u v, v)\right)
$$

seja $k$ o maior inteiro tal que $u^{k} \mid \tilde{X}_{u}$ e $u^{k} \mid \tilde{X}_{v}$. Defina $\bar{X}(u, v)=\left(\bar{X}_{u}, \bar{X}_{v}\right)=\frac{\tilde{X}(u v, v)}{v^{k}}$. Neste caso, a singularidade $(0,0)$ é levada para a curva $v=0$ e, para $v=0$ as singularidades de $\tilde{X}$ são determinadas pelas soluções de $\bar{X}_{u}=0$.

Exemplo 1.4.5. Considere o campo $X=\left(x^{2}-2 x y, y^{2}-2 x y\right)$, com singularidade isolada em $(0,0)$.

Aplicando blow up na direção $x$ em $(0,0), x=u$ e $y=u v$, obtemos:

$$
\tilde{X}=\left(\tilde{X}_{u}, \tilde{X}_{v}\right)=\left(u^{2}-2 u^{2} v, 3 u v^{2}-3 u v\right)
$$

Como $k=1$ é o maior inteiro tal que $u^{k} \mid \tilde{X}_{u}$ e $u^{k} \mid \tilde{X}_{v}$, temos:

$$
\bar{X}(u, v)=\frac{\tilde{X}(u, u v)}{u}=\left(\bar{X}_{u}, \bar{X}_{v}\right)=\left(u-2 u v, 3 v^{2}-3 v\right)
$$


Em $u=0$, temos $v=0$ e $v=1$ como raizes de $\bar{X}_{v}=0$. Como

$$
J \bar{X}(u, v)=\left(\begin{array}{cc}
1-2 v & -2 u \\
0 & 6 v-3
\end{array}\right)
$$

temos det $J \bar{X}(0,0)=\operatorname{det} J \bar{X}(0,1)=-3$ e portanto, as singularidades $(0,0)$ e $(0,1)$ são do tipo sela.

Agora, aplicamos um blow up na direção $y, x=u v$ e $y=v$, obtendo:

$$
\tilde{X}=\left(\tilde{X}_{u}, \tilde{X}_{v}\right)=\left(3 u^{2} v-3 u v, v^{2}-2 u v^{2}\right)
$$

Como $k=1$ é o maior inteiro tal que $v^{k} \mid \tilde{X}_{u}$ e $v^{k} \mid \tilde{X}_{v}$, temos:

$$
\bar{X}(u, v)=\frac{\tilde{X}(u, u v)}{v}=\left(\bar{X}_{u}, \bar{X}_{v}\right)=\left(3 u^{2} v-3 u v, v^{2}-2 u v^{2}\right) .
$$

Em $v=0$, temos $u=0$ e $u=1$ como raizes de $\bar{X}_{u}=0$. Como

$$
J \bar{X}(u, v)=\left(\begin{array}{cc}
6 u-3 & 0 \\
-2 v & 1-2 u
\end{array}\right)
$$

temos det $J \bar{X}(0,0)=\operatorname{det} J \bar{X}(1,0)=-3$ e deste modo, as singularidades $(0,0)$ e $(1,0)$ são do tipo sela.

A figura abaixo ilustra os blow ups direcionais em $x$ e y efetuados neste exemplo: 

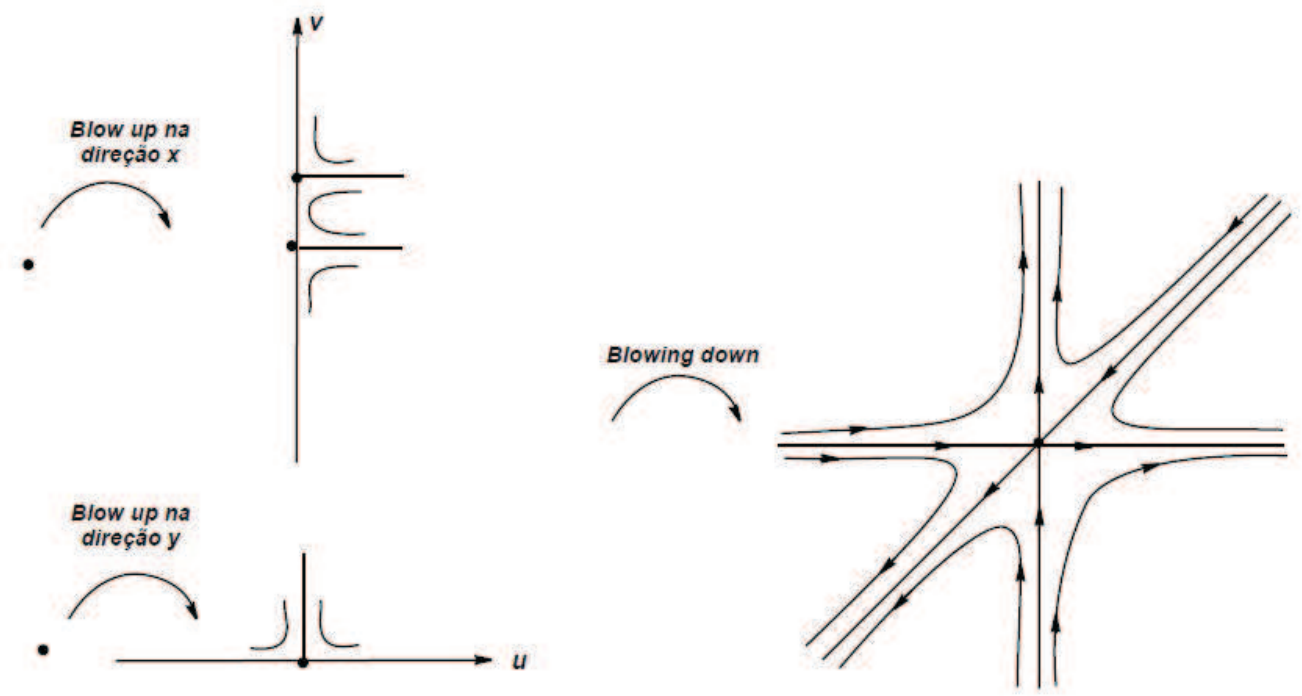

Figura 1.4: Exemplo de blow up direcional

Observe que o esboço do retrato de fase acima, obtido com blow up direcional, é exatamente o mesmo retrato obtido por blow up polar no Exemplo 1.4.4.

O Exemplo 1.4.5 motiva uma pergunta natural: qual é a relação entre blow up polar e blow up direcional?

A seguir, respondemos a esta pergunta.

\section{Relação entre blow up polar e blow up direcional}

Seja $F: \mathbb{R} \times\left(-\frac{\pi}{2}, \frac{\pi}{2}\right) \rightarrow \mathbb{R}^{2}$, dada por $F(r, \theta)=(r \cos (\theta), \operatorname{tg}(\theta))$. Então,

$$
J F(r, \theta)=\left(\begin{array}{cc}
\cos (\theta) & -r \operatorname{sen}(\theta) \\
0 & \sec ^{2}(\theta)
\end{array}\right) \Rightarrow \operatorname{det} J F(r, \theta)=\sec (\theta) \neq 0, \forall \theta \in\left(-\frac{\pi}{2}, \frac{\pi}{2}\right)
$$

Consequentemente, $F(r, \theta)$ é difeomorfismo.

Além disso:

(i) $F$ leva $\left\{(r, \theta) ; r=0\right.$ e $\left.-\frac{\pi}{2}<\theta<\frac{\pi}{2}\right\}$ sobre o eixo $O_{y}$. Em particular, para $\theta=0$, temos $F(r, 0)=(0,0) \in O_{x y}$; 
(ii) $J F(0,0)=\left(\begin{array}{ll}1 & 0 \\ 0 & 1\end{array}\right)$. Portanto, $F$ preserva a parte linear do campo;

(iii) $F(r, \theta)=\left(x, \frac{y}{x}\right)$. Então, $F^{-1}(u, v)=F^{-1}\left(x, \frac{y}{x}\right)=(r, \theta)$, pois

$$
\left\{\begin{array} { l } 
{ u = r \operatorname { c o s } ( \theta ) } \\
{ v = \operatorname { t g } ( \theta ) }
\end{array} \Rightarrow \left\{\begin{array}{l}
u=r \cos (\theta)=x \\
u v=r \operatorname{sen}(\theta)=y
\end{array}\right.\right.
$$

Considere o campo vetorial apresentado no Exemplo 1.4.5. Quando aplicamos o blow up na direção $x$, obtivemos as singularidades $(0,1)$ e $(0,0)$, que são do tipo sela.

Assim: $\left\{\begin{aligned}(u, v) & \rightarrow(r, \theta) \\ (0,0) & \rightarrow(0,0) \\ (0,1) & \rightarrow\left(0, \frac{\pi}{4}\right)\end{aligned}\right.$

Por simetria, temos $\pi$ e $\frac{5 \pi}{4}$ singularidades do tipo sela, como podemos observar figura 1.5:

Blow Up na direção $\mathrm{x}$

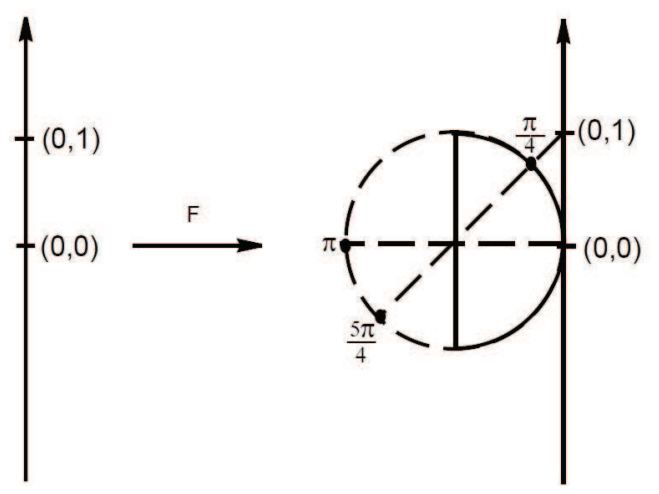

Figura 1.5: Relação entre blow up na direção x e blow up polar

Seja $G:(0, \pi) \times \mathbb{R} \rightarrow \mathbb{R}^{2}$, dada por $G(\theta, r)=(\cot g(\theta), r \operatorname{sen}(\theta))$. Então,

$J G(\theta, r)=\left(\begin{array}{cc}-\operatorname{cossec}(\theta) & 0 \\ r \cos (\theta) & \operatorname{sen}(\theta)\end{array}\right) \Rightarrow \operatorname{det} J G(\theta, r)=-\operatorname{cossec}(\theta) \neq 0, \forall \theta \in(0, \pi)$.

Consequentemente, $G(r, \theta)$ é difeomorfismo. Além disso: 
(i) $G$ leva $\{(\theta, r) ; r=0$ e $0<\theta<\pi\}$ sobre o eixo $O_{x}$. Em particular, para $\theta=\frac{\pi}{2}$, temos $G\left(\frac{\pi}{2}, 0\right)=(0,0) \in O_{x y}$;

(ii) $J G\left(\frac{\pi}{2}, 0\right)=\left(\begin{array}{cc}-1 & 0 \\ 0 & 1\end{array}\right)$. Logo, $G$ preserva a parte linear do campo;

(iii) $G(\theta, r)=\left(x, \frac{x}{y}\right)$. Então, $G^{-1}(u, v)=G^{-1}\left(\frac{x}{y}, y\right)=(r, \theta)$, pois

$$
\left\{\begin{array} { l } 
{ u = \operatorname { c o t g } ( \theta ) } \\
{ v = r \operatorname { s e n } ( \theta ) }
\end{array} \Rightarrow \left\{\begin{array}{l}
u v=r \cos (\theta)=x \\
v=r \operatorname{sen}(\theta)=y
\end{array}\right.\right.
$$

Considere novamente o campo vetorial apresentado no Exemplo 1.4.5. Aplicando blow up na direção $y$, obtivemos as singularidades $(1,0)$ e $(0,0)$, que são do tipo sela.

$$
\text { Assim: }\left\{\begin{aligned}
(u, v) & \rightarrow(\theta, r) \\
(0,0) & \rightarrow\left(\frac{\pi}{2}, 0\right) \\
(1,0) & \rightarrow\left(\frac{\pi}{4}, 0\right)
\end{aligned}\right.
$$

Por simetria, temos $\frac{3 \pi}{2}$ e $\frac{5 \pi}{4}$ singularidades do tipo sela, como podemos observar figura 1.6:

\section{Blow Up na direção y}

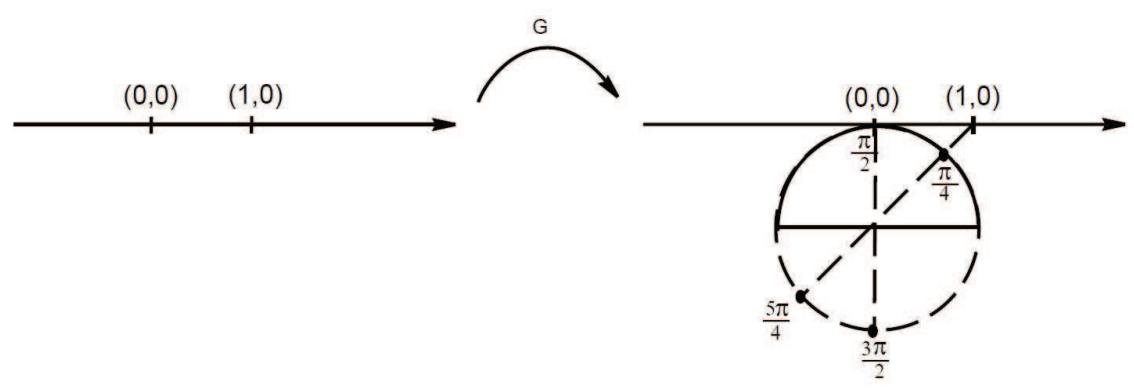

Figura 1.6: Relação entre blow up na direção y e blow up polar

A escolha por uma das técnicas, blow up polar ou blow up direcional fica a critério de cada pesquisador. Entretanto, em geral, para blow ups sucessivos é mais conveniente utilizar o blow up direcional, como será ilustrado no próximo exemplo.

Exemplo 1.4.6. Seja o campo $X=\left(y^{3}, x+y^{2}+y^{3}\right)$, com singularidade isolada em $(0,0)$. 
Vamos aplicar blow up na direção $x$ em $(0,0)$. Através da mudança de coordenadas $x=u$ e $y=u v$, obtemos $\tilde{X}=\left(\tilde{X}_{u}, \tilde{X}_{v}\right)=\left(u^{3} v^{3}, 1+u v^{2}+u^{2} v^{3}-u^{2} v^{4}\right)$.

Neste caso, não existe $k \in \mathbb{N}$ tal que $u^{k} \mid \tilde{X}_{u}$ e $u^{k} \mid \tilde{X}_{v}$. Portanto, $\bar{X}=\tilde{X}$.

Ao longo de $u=0$, temos $\tilde{X}_{v}=1$. Logo, não existe singularidade na direção de $x$.

Aplicando blow up na direção $y, x=u v$ e $y=v$, obtemos $\tilde{X}=\left(\tilde{X}_{u}, \tilde{X}_{v}\right)=\left(v^{2}-u^{2}-\right.$ $\left.u v-u v^{2}, u v+v^{2}+v^{3}\right)$.

Como não existe $k \in \mathbb{N}$ tal que $u^{k} \mid \tilde{X}_{u}$ e $u^{k} \mid \tilde{X}_{v}$, temos $\bar{X}=\tilde{X}$.

Em $v=0$, temos $u=0$ como solução de $\tilde{X}_{u}=0$. Além disso,

$$
J \bar{X}(x, y)=\left(\begin{array}{cc}
-2 u-v-v^{2} & 2 v-u-2 u v \\
v & u+2 v+3 v^{3}
\end{array}\right) \Rightarrow J \bar{X}(0,0)=\left(\begin{array}{ll}
0 & 0 \\
0 & 0
\end{array}\right) .
$$

Assim, (0,0) é uma singularidade não hiperbólica de $\bar{X}$. Aplicando novo blow up na direção de $v$ em $(0,0), u=w z$ e $v=z$, temos $\tilde{X}=\left(\tilde{X}_{w}, \tilde{X}_{z}\right)=\left(z-2 w^{2} z-2 w z-\right.$ $\left.2 w z^{2}, w z^{2}+z^{2}+z^{3}\right)$.

$k=1$ é o maior inteiro tal que $z^{k} \mid \tilde{X}_{w}$ e $z^{k} \mid \tilde{X}_{z}$.

Assim, $\bar{X}=\frac{\tilde{X}}{z}=\left(\bar{X}_{w}, \bar{X}_{z}\right)=\left(1-2 w^{2}-2 w-2 w z, w z+z+z^{2}\right)$.

$E m z=0$, as soluções de $\bar{X}_{w}=0$ são $\frac{-2-\sqrt{12}}{4} e \frac{-2+\sqrt{12}}{4}$.

A parte linear de $\bar{X}$ é dada por:

$J \bar{X}(x, y)=\left(\begin{array}{cc}-4 w-2 & -2 w \\ z & w+2 z+1\end{array}\right) \Rightarrow\left\{\begin{array}{c}\operatorname{det} J \bar{X}\left(0, \frac{-2-\sqrt{12}}{4}\right)=\frac{\sqrt{12}}{2}-3<0 \\ \operatorname{det} J \bar{X}\left(0, \frac{-2+\sqrt{12}}{4}\right)=-\frac{\sqrt{12}}{2}-3<0 .\end{array}\right.$

Consequentemente, as singularidades $\left(0, \frac{-2-\sqrt{12}}{4}\right) e\left(0, \frac{-2+\sqrt{12}}{4}\right)$ são do tipo sela. 
A figura 1.7 apresenta as etapas para obtermos o esboço do retrato de fase de $X$ numa vizinhança da origem.
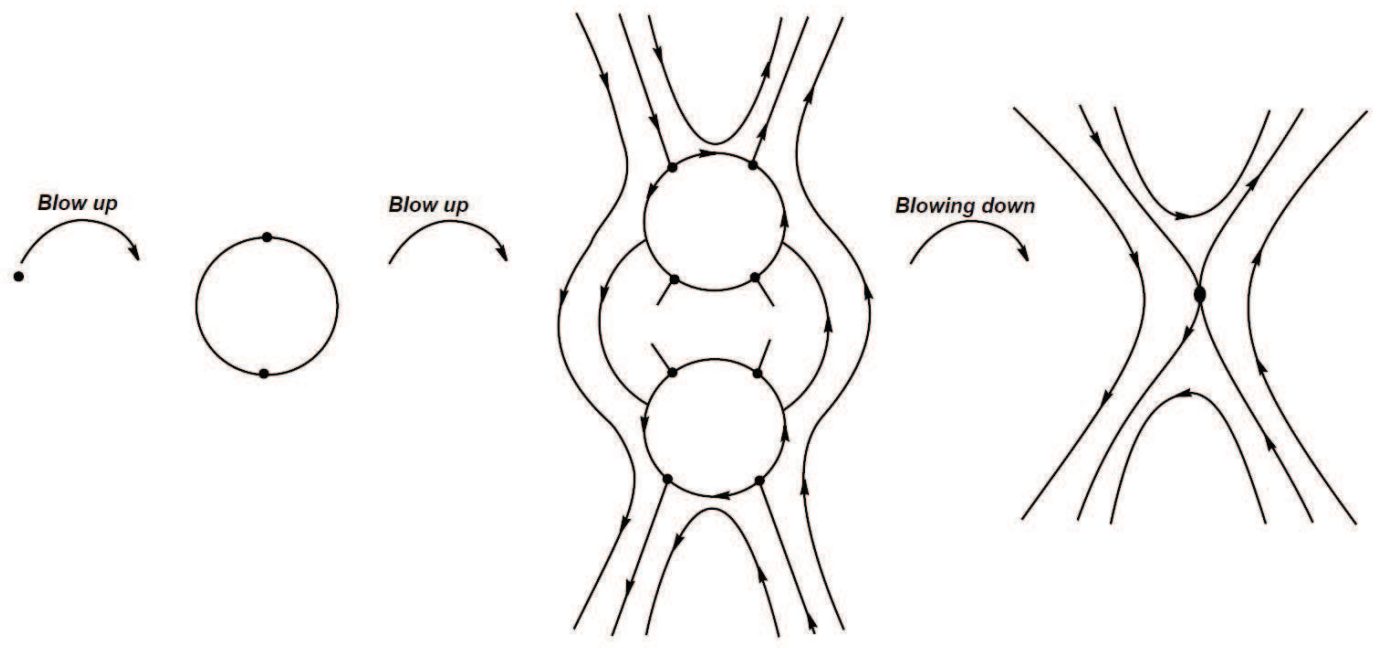

Figura 1.7: Exemplo de blow ups direcionais sucessivos

\section{Blow-up (m, n)-quasehomogêneo}

A técnica de blow-up $(m, n)$-quasehomogêneo que apresentaremos nesta seção é uma generalização da técnica de blow-up polar apresentada anteriormente. De fato, em muitos casos, ao invés de uma desnecessária limitação na escolha do blow-up polar, é mais conveniente "adaptar" a técnica ao campo vetorial considerado, utilizando-se o blow-up $(m, n)$ quasehomogêneo. Este método foi aplicado pela primeira vez por Lyapunov - cf. [37], e se constitui em importante ferramenta na investigação do problema do centro-foco em diversos trabalhos recentes - cf. [1, 2, 23].

Definição 1.4.7. Sejam a aplicação $f: \mathbb{R}^{n} \rightarrow \mathbb{R} e\left(\alpha_{1}, \ldots, \alpha_{n}\right) \in \mathbb{N}_{+}^{n}, k \in \mathbb{N}$, tais que

$$
f\left(t^{\alpha_{1}} x_{1}, \ldots, t^{\alpha_{n}} x_{n}\right)=t^{k} f\left(x_{1}, \ldots, x_{n}\right), \forall t \in \mathbb{R}
$$

Então, $f$ é chamada de função $\left(\alpha_{1}, \ldots, \alpha_{n}\right)$-quasehomogênea de grau $k$.

Definição 1.4.8. Seja $X$ um campo vetorial da forma $\left.X=f_{1} \frac{\partial}{\partial x_{1}}+\ldots+f_{n} \frac{\partial}{\partial x_{n}}\right)$, com $f_{j}\left(x_{1}, \ldots, x_{n}\right), j=1, \ldots, n$, funções $\left(\alpha_{1}, \ldots, \alpha_{n}\right)$-quasehomogêneas de grau $k+\alpha_{j}$. Então, $X$ é denominado um campo vetorial $\left(\alpha_{1}, \ldots, \alpha_{n}\right)$-quasehomogêneo de grau $k$. 
Lema 1.4.9. Seja $\left(\alpha_{1}, \ldots, \alpha_{n}\right) \in \mathbb{N}_{+}^{n}$ e suponha que os $\alpha_{j}$ sejam primos todos entre si. Então, é sempre possível decompor um campo vetorial em $\mathbb{R}^{n}$ em uma série formal centrada em $0 \in \mathbb{R}^{n}, X=\sum_{j \geq k} X_{j}$, onde cada $X_{j}$ é um campo vetorial $\left(\alpha_{1}, \ldots, \alpha_{n}\right)$-quasehomogêneo de grau $j . k$ é o primeiro inteiro tal que $X_{k} \neq 0$ e é denominado de ordem do campo $X$.

A demonstração deste lema pode ser encontrada em [13].

No caso de campos vetoriais planares, apresentamos os seguintes resultados.

Definição 1.4.10. Seja um campo vetorial $(m, n)$-quasehomogêneo, $m, n \in \mathbb{N}_{+}$. Introduzimos, então, as coordenadas $(m, n)$-quasehomogêneas: $x=r^{m} \operatorname{Cs}(\theta), y=r^{n} \operatorname{Sn}(\theta)$, onde as funções Cs e Sn são definidas como sendo a única solução do Problema de Cauchy

$$
\left\{\begin{array}{l}
\dot{x}=\frac{d x}{d \theta}=-y^{2 m-1}, \\
\dot{y}=\frac{d y}{d \theta}=x^{2 n-1},
\end{array}\right.
$$

com condições iniciais $x(0)=1, y(0)=0$.

Observe que, por definição, Sn e Cs são funções $T$-periódicas, onde

$$
T=\frac{2 m^{(1-2 m) / 2 m}}{n^{1 / 2 m}} \int_{0}^{1}(1-t)^{(1-2 m) / 2 m} t^{(1-2 n) / 2 n} d t
$$

Além disso, $n \operatorname{Sn}^{2 m}(\theta)+m \operatorname{Cs}^{2 n}(\theta)=m$.

Considere a relação de equivalência $x \sim y$, se $x-y=n T, n \in \mathbb{Z}$ e seja $S_{T}^{1}$ o conjunto das classes de equivalência assim obtidas. Para um campo vetorial analítico planar $X$, com $X(0,0)=0$, seja $k \geq 0$ a ordem de $X$ com respeito à decomposição do tipo $(m, n)$. Então, $\tilde{X}$ é o único campo vetorial definido pelo diagrama comutativo:

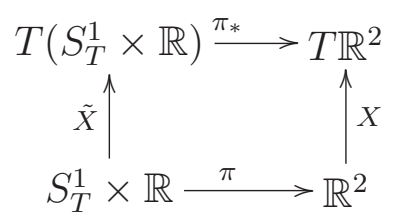

onde $\pi: S_{T}^{1} \times \mathbb{R} \rightarrow \mathbb{R}^{2}$ é a mudança de coordenadas $(m, n)$-quasehomogênea definida acima, para $(\theta, r) \in S_{T}^{1} \times \mathbb{R}$. A aplicação $\pi$ é sobrejetora e própria. Além disso, $\pi^{-1}(0)=$ $S_{T}^{1} \times\{0\}$ e a restrição de $\pi$ a $S_{T}^{1} \times(\mathbb{R} \backslash\{0\})$ é um difeomorfismo sobre sua imagem. 
É possível dividir $\tilde{X}$ por $r^{k}$ - cf. [13]. Seja $\bar{X}$ o campo vetorial resultante. Sua expressão é $\bar{X}(\theta, r)=(P(\theta, r), Q(\theta, r))$, onde $P(\theta, r)=\sum_{j \geq k} \frac{1}{m}\left[\operatorname{Cs}^{2 n-1}(\theta) P_{j}(\operatorname{Cs}(\theta), \operatorname{Sn}(\theta))+\operatorname{Sn}^{2 m-1}(\theta) Q_{j}(\operatorname{Cs}(\theta), \operatorname{Sn}(\theta))\right] r^{j-k+1}$, $Q(\theta, r)=\sum_{j \geq k}\left[\operatorname{Cs}(\theta) Q_{j}(\operatorname{Cs}(\theta), \operatorname{Sn}(\theta))-\frac{n}{m} \operatorname{Sn}(\theta) P_{j}(\operatorname{Cs}(\theta), \operatorname{Sn}(\theta))\right] r^{j-k}, \mathrm{e}$ $\left.X_{j}(x, y)=P_{j}(x, y) \frac{\partial}{\partial x}+Q_{j}(x, y)\right) \frac{\partial}{\partial y}$.

No caso em que $(m, n)=(1,1)$, a técnica se reduz ao blow-up polar, $\operatorname{com} \operatorname{Sn}(\theta)=$ $\operatorname{sen}(\theta), \operatorname{Cs}(\theta)=\cos (\theta)$ e $T=2 \pi$.

O exemplo a seguir, extraído de [13], ilustra o método.

Exemplo 1.4.11. O campo vetorial planar $X(x, y)=\left(y, x^{2}\right)$ é $(2,3)$-quasehomogêneo de grau $k=1$. Assim,

$$
\begin{aligned}
\bar{X}(\theta, r) & :=\left(\bar{X}_{r}(\theta, r), \bar{X}_{\theta}(\theta, r)\right) \\
& =\left(\frac{1}{2} \operatorname{Cs}^{2}(\theta) \operatorname{Sn}(\theta)\left(\operatorname{Cs}^{3}(\theta)+\operatorname{Sn}^{2}(\theta)\right) r, \operatorname{Cs}^{3}(\theta)-\frac{3}{2} \operatorname{Sn}^{2}(\theta)\right)
\end{aligned}
$$

Logo, $\bar{X}(\theta, 0)=\left(0, \operatorname{Cs}^{3}(\theta)-\frac{3}{2} \operatorname{Sn}^{2}(\theta)\right)$ tem duas singularidades em $\theta= \pm \theta_{0}$, onde $\theta_{0}$ é solução de $\operatorname{Sn}\left(\theta_{0}\right)=\sqrt{\frac{2}{3} \operatorname{Cs}^{3}\left(\theta_{0}\right)}, \operatorname{Cs}\left(\theta_{0}\right)>0$. Em tais pontos, vale sgn $\left(\frac{\partial \bar{X}_{r}(\theta, r)}{\partial r}\right)=$ $\operatorname{sgn}\left(\operatorname{Sn}\left(\theta_{0}\right)\right)$, onde sgn é a função sinal.

Um esboço do retrato de fase do campo é dado na figura a seguir:

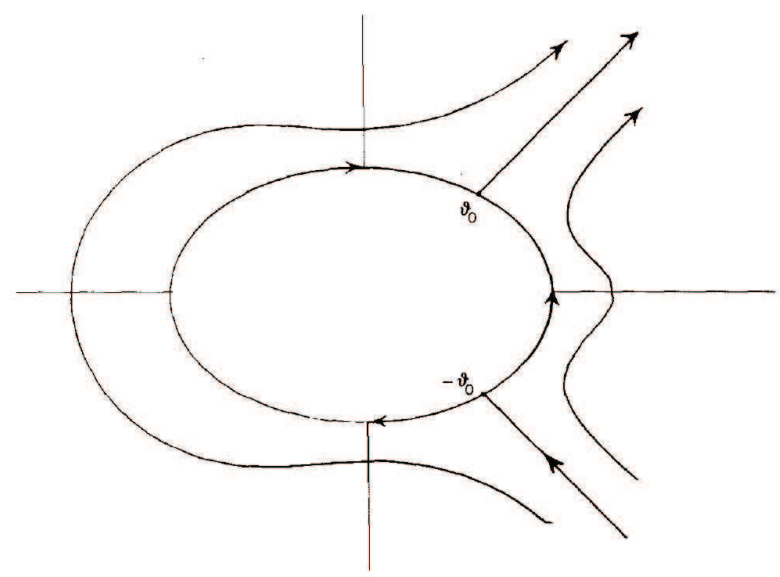

Figura 1.8: Exemplo de blow-up (m,n)-quasehomogêneo 
O blow-up $(1, n)$-quasehomogêneo será ferramenta importante na Seção 3.1. Por esta razão, discutiremos o método em detalhes.

Proposição 1.4.12. [25] Sejam $x(\theta)=\operatorname{Cs}(\theta), y(\theta)=\operatorname{Sn}(\theta)$ conforme Definição 1.4.10, para $m=1$ e $n \in \mathbb{N}$. Então valem as seguintes propriedades:

1. $\operatorname{Cs}^{2 n}(\theta)+n \operatorname{Sn}^{2}(\theta)=1$;

2. $\operatorname{Cs}(\theta)$ e $\operatorname{Sn}(\theta)$ são funções T-periódicas, onde $T=2 \sqrt{\frac{\pi}{n}} \frac{\Gamma\left(\frac{1}{2 n}\right)}{\Gamma\left(\frac{n+1}{2 n}\right)}$;

3. $\int_{0}^{\theta} \operatorname{Sn}(\varphi) \operatorname{Cs}^{q}(\varphi) d \varphi=-\frac{\operatorname{Cs}^{q+1}(\theta)}{q+1}$;

4. $\int_{0}^{\theta} \operatorname{Sn}^{p}(\varphi) \operatorname{Cs}^{2 n-1}(\varphi) d \varphi=\frac{\operatorname{Sn}^{p+1}(\theta)}{p+1}$;

5. $\int_{0}^{\theta} \operatorname{Sn}^{p}(\varphi) \operatorname{Cs}^{q}(\varphi) d \varphi=-\frac{\operatorname{Sn}^{p-1}(\theta) \operatorname{Cs}^{q+1}(\theta)}{(p-1) n+q+1}+\frac{p-1}{(p-1) n+q+1} \int_{0}^{\theta} \operatorname{Sn}^{p-2}(\varphi) \operatorname{Cs}^{q}(\varphi) d \varphi ;$

6. $\int_{0}^{\theta} \operatorname{Sn}^{p}(\varphi) \operatorname{Cs}^{q}(\varphi) d \varphi=\frac{n \operatorname{Sn}^{p+1}(\theta) \operatorname{Cs}^{q-2 n+1}(\theta)}{(p-1) n+q+1}+\frac{q-2 n+1}{(p-1) n+q+1} \int_{0}^{\theta} \operatorname{Sn}^{p}(\varphi) \operatorname{Cs}^{q-2 n}(\varphi) d \varphi ;$

7. $\int_{0}^{T} \operatorname{Sn}^{p}(\varphi) \operatorname{Cs}^{q}(\varphi) d \varphi=0$, se p ou q é ímpar;

8. $\int_{0}^{T} \operatorname{Sn}^{p}(\varphi) \operatorname{Cs}^{q}(\varphi) d \varphi=\frac{2}{\sqrt{n^{p+1}}} \frac{\Gamma\left(\frac{p+1}{2}\right) \Gamma\left(\frac{q+1}{2 n}\right)}{\Gamma\left(\frac{p+1}{2}+\frac{q+1}{2 n}\right)}$, se $p$ e $q$ forem ambos números pares.

A demonstração destas propriedades pode ser encontrada em [37] e [25].

Dos resultados acima, e tomando um número natural $n \in \mathbb{N}$, podemos introduzir as coordenadas $(1, n)$-quasehomogêneas como sendo:

$$
\left\{\begin{array}{l}
x=r \operatorname{Cs}(\theta), \\
y=r^{n} \operatorname{Sn}(\theta) .
\end{array}\right.
$$

onde as funções Sn $e$ Cs são dadas em (1.4.6), para $m=1$ e $n \in \mathbb{N}$.

Do primeiro item da Proposição 1.4.12, segue que $x^{2 n}+n y^{2}=r^{2 n}$. Derivando-se as 
expressões em (1.4.7), obtemos

$$
\begin{aligned}
& \dot{x}=\dot{r} \operatorname{Cs}(\theta)-\dot{\theta} r \operatorname{Sn}(\theta) \\
& \dot{y}=n r^{n-1} \dot{r} \operatorname{Sn}(\theta)+\dot{\theta} r^{n} \mathrm{Cs}^{2 n-1}(\theta)
\end{aligned}
$$

Multiplicando-se a primeira equação por $\left[r^{2 n-1} \operatorname{Cs}^{2 n-1}(\theta)\right]$ e a segunda por $\left[r^{n} \operatorname{Sn}(\theta)\right]$ e somando membro a membro obtemos

$$
r^{2 n-1} \operatorname{Cs}^{2 n-1}(\theta) \dot{x}+r^{n} \operatorname{Sn}(\theta) \dot{y}=r^{2 n-1}\left(\operatorname{Cs}^{2 n}(\theta)+n \operatorname{Sn}^{2}(\theta)\right) \dot{r} .
$$

Usando (1.4.7), temos

$$
x^{2 n-1} \dot{x}+y \dot{y}=r^{2 n-1} \dot{r} .
$$

Portanto,

$$
\dot{r}=\frac{x^{2 n-1} \dot{x}+y \dot{y}}{r^{2 n-1}} .
$$

Fazendo o produto da primeira equação com $\left[n r^{n-1} \operatorname{Sn}(\theta)\right]$ e da segunda com $[-\operatorname{Cs}(\theta)]$ e efetuando cálculos similares, obtemos

$$
\dot{\theta}=\frac{x \dot{y}-n y \dot{x}}{r^{n+1}} .
$$

Deste modo, temos o sistema em coordenadas $r$ e $\theta$ :

$$
\left\{\begin{array}{l}
\dot{r}=\frac{x^{2 n-1} \dot{x}+y \dot{y}}{r^{2 n-1}}, \\
\dot{\theta}=\frac{x \dot{y}-n y \dot{x}}{r^{n+1}}
\end{array}\right.
$$

No Captítulo 3, retomaremos o tema blow up na prova de um dos teoremas fundamentais desta dissertação. 


\section{Capítulo}

\section{Problema do centro-foco}

Considere o sistema diferencial analítico no plano

$$
\left\{\begin{array}{l}
\dot{x}=P(x, y), \\
\dot{y}=Q(x, y),
\end{array}\right.
$$

e $X=P(x, y) \frac{\partial}{\partial x}+Q(x, y) \frac{\partial}{\partial x}$ o campo vetorial associado ao sistema (2.0.1). Seja $p$ um ponto singular de $X$.

Como vimos, o problema do centro-foco pode ser dividido em três casos distintos, segundo o tipo do ponto singular do sistema diferencial analisado: não degenerado, degenerado linearmente nulo, ou nilpotente. Neste capítulo, discutiremos o caso não degenerado, apresentando alguns resultados importantes tais como o Teorema de Bautin, e discutiremos brevemente o caso linearmente nulo.

\subsection{Singularidades não degeneradas}

Sabemos da teoria qualitativa de sistemas diferenciais planares que, se os autovalores da matriz $D X(p)$ são complexos conjugados não nulos $\alpha \pm i \beta$, então o ponto singular $p$ é monodrômico - cf. [44, 22]. 
Se o traço de $D X(p)$ for não nulo, então $p$ é um foco (estável se $\alpha<0$ e instável caso contrário).

Se o traço de $D X(p)$ for nulo, então os autovalores são números imaginários puros, e assim, $p$ pode ser um foco ou um centro de $X$. O problema que visa distinguir entre esses dois tipos de singularidades no caso de autovalores puramente imaginários é o clássico problema do centro-foco de Lyapunov-Poincaré, ou simplesmente problema do centro-foco para pontos singulares não degenerados.

Sob o ponto de vista teórico, o problema de encontrar condições necessárias para um centro é solucionado pela determinação das constantes de Lyapunov. Apresentamos a seguir, os principais resultados relacionados a este assunto.

\subsubsection{Preâmbulo teórico}

Nesta seção, desenvolveremos algumas ferramentas úteis na investigação de importantes propriedades da aplicação de Poincaré e da função sucessão. Em particular, obteremos uma mudança de coordenadas que será utilizada na demonstração da Proposição (2.1.5), que por sua vez, permitirá calcular a expressão da derivada da aplicação de Poincaré na origem.

Seja o sistema diferencial analítico planar da forma (2.0.1), e seja $\gamma_{0}$ uma órbita fechada de período $\tau>0$ deste sistema, parametrizada por

$$
\left\{\begin{array}{l}
x=\varphi(t), \\
y=\psi(t),
\end{array} t \in \mathbb{R}^{+}\right.
$$

Como $\gamma_{0}$ é periódica de período $\tau, \varphi, \psi$ também são funções periódicas de período $\tau$. Além disso, $\varphi, \psi$, sendo soluções do sistema analítico (2.0.1), são também analíticas, e particularmente, possuem segundas derivadas contínuas.

Por cada ponto $M=(\varphi(s), \psi(s))$ da curva $\gamma_{0}$, construímos uma seção transversal contida na reta normal ao ponto $M$, com comprimento $\delta \sqrt{\varphi^{\prime}(s)^{2}+\psi^{\prime}(s)^{2}}, \delta>0$, em ambos os lados de $\gamma_{0}$ ao longo da normal.

Tomamos então $\delta>0$ suficientemente pequeno de forma a satisfazer o Lema 2.1.6. Então, as extremidades internas (externas) em relação à curva $\gamma_{0}$, das seções transversais 
de comprimento $\delta \sqrt{\varphi^{\prime}(s)^{2}+\psi^{\prime}(s)^{2}}$, contidas nas retas normais a cada ponto de $\gamma_{0}$, formam uma curva simples $\Gamma_{1}\left(\Gamma_{2}\right)$, como ilustrado na figura a seguir.

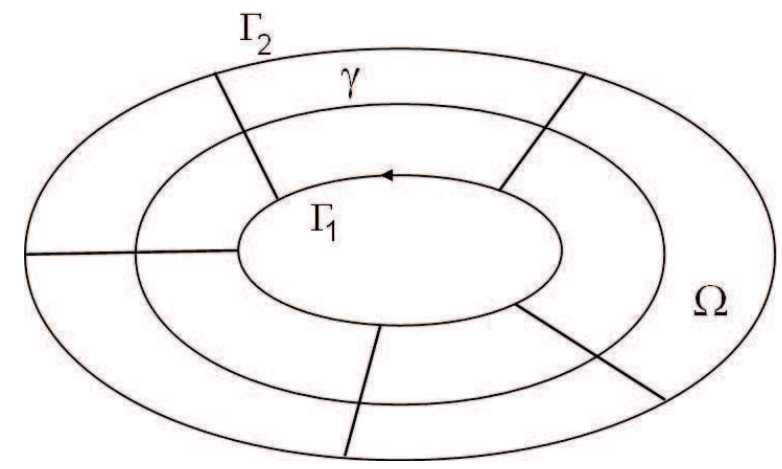

Figura 2.1: Órbita periódica $\gamma_{0}$, curvas fechadas simples $\Gamma_{1}$ e $\Gamma_{2}$ e a região $\Omega$

As curvas $\Gamma_{1}$ e $\Gamma_{2}$ então, são tais que definem uma região $\Omega$ no plano contendo inteiramente $\gamma_{0}$. Observe que $\Omega$ é claramente homeomorfa a um anel circular.

Para $\delta>0$ suficientemente pequeno, o sistema (2.0.1) não tem singularidades em $\Omega$, pois a origem é uma singularidade isolada.

Considere as funções:

$$
\left\{\begin{array}{l}
\bar{\varphi}(s, n)=\varphi(s)+n \psi^{\prime}(s), \\
\bar{\psi}(s, n)=\psi(s)-n \varphi^{\prime}(s) .
\end{array}\right.
$$

definidas na faixa

$$
\left\{(s, n) \in \mathbb{R}^{2} \mid s \in(-\infty,+\infty), n \in(-\delta, \delta)\right\} .
$$

onde $s$ indica o tempo e $\psi^{\prime}(s)$ denota $\frac{d \psi}{d s}$.

As funções $\bar{\varphi}$ e $\bar{\psi}$ possuem as seguintes propriedades:

1. $\bar{\varphi}$ e $\bar{\psi}$ são funções analíticas, uma vez que $\varphi, \psi$ são analíticas;

2. $\bar{\varphi}$ e $\bar{\psi}$ e suas respectivas derivadas parciais são periódicas em $s$, de período $\tau$;

3. $\bar{\varphi}(s, 0)=\varphi(s)$

$\bar{\psi}(s, 0)=\psi(s)$

Ou seja, para $n=0, x=\bar{\varphi}(s, n)$ e $y=\bar{\psi}(s, n)$ são as equações paramétricas de $\gamma_{0}$, onde o parâmetro $s$ coincide com $t$. 
4. O determinante

$$
\triangle(s, n)=\frac{D(\bar{\varphi}, \bar{\psi})}{D(s, n)}=\left|\begin{array}{cc}
\bar{\varphi}_{s}^{\prime}(s, n) & \bar{\varphi}_{n}^{\prime}(s, n) \\
\bar{\psi}_{s}^{\prime}(s, n) & \bar{\psi}_{n}^{\prime}(s, n)
\end{array}\right|
$$

para $n=0$ é igual a $\triangle(s, 0)=-\varphi^{\prime}(s)^{2}-\psi^{\prime}(s)^{2} \neq 0$, pois $\varphi$ e $\psi$ parametrizam a solução periódica $\gamma_{0}$ em $(2.0 .1)$.

Ou seja, para $n=0$, o determinante $\triangle(s, n)$ não se anula para qualquer $s,-\infty<$ $s<+\infty$.

Observamos que $\bar{\varphi}_{s}^{\prime}(s, n)$ denota $\frac{\partial \bar{\varphi}(s, n)}{\partial s}$, o mesmo valendo para as demais derivadas parciais expressas acima.

Da propriedade (4), do fato do determinante ser uma função contínua, da compacidade do segmento $0 \leq s \leq \tau$ e usando a periodicidade das funções $\bar{\varphi}$ e $\bar{\psi}$ em $s$, segue que para valores suficientemente pequenos de $n, \triangle(s, n) \neq 0$.

Por fim, observamos que, se $\delta>0$ é suficientemente pequeno, todos os segmentos normais a pontos distintos de $\gamma_{0}$ são seções transversais para as trajetórias de (2.0.1) contidas na região $\Omega$ - cf. [5].

Em resumo, $\delta>0$ precisa ser suficientemente pequeno para satisfazer as seguintes condições:

1. Os segmentos de reta de comprimento $2 \delta \sqrt{\varphi^{\prime}(s)^{2}+\psi^{\prime}(s)^{2}}$, normais à solução periódica $\gamma_{0}$ em pontos distintos não se interceptam. Disto segue que, em particular, a região $\Omega$ é homeomorfa a um anel circular;

2. Todos os segmentos normais descritos no item anterior são seções transversais para pontos pertencentes a trajetórias do sistema na região $\Omega$;

3. Não existem singularidades de (2.0.1) em $\Omega$;

4. O determinante $\triangle(s, n)$ é não nulo na faixa (2.1.1).

Mostraremos agora, que

$$
\left\{\begin{array}{l}
x=\bar{\varphi}(s, n) \equiv \varphi(s)+n \psi^{\prime}(s), \\
y=\bar{\psi}(s, n) \equiv \psi(s)-n \varphi^{\prime}(s),
\end{array}\right.
$$


é uma mudança de coordenadas local do sistema (2.0.1). Para tanto, é suficiente mostrar que (2.1.2) é um homeomorfismo.

De fato:

(i) $n=0$ é levado por (2.1.2) na curva $\gamma_{0}$. As retas $n=c, 0<|c|<\delta$, paralelas ao eixo $s$ são levadas por (2.1.2) em curvas fechadas simples que não se interceptam, ficando uma "contida" na outra, em $\Omega$;

(ii) os pontos da forma $\left\{(s, n) \in \mathbb{R}^{2} \mid s=s_{0}, n \in(-\delta, \delta)\right\}$ são levados por (2.1.2) em segmentos normais a $\gamma_{0}$;

(iii) Dos dois itens anteriores, segue que (2.1.2) leva a faixa (2.1.1) sobrejetivamente na região $\Omega$. De fato, $\forall(x, y) \in \Omega,(x, y)$ é a intersecção de uma curva fechada simples $\Gamma_{0}$ e de um segmento $\Sigma_{0}$ normal a $\Omega$. Tome $n=c$ e $s=s_{0}$ de tal forma que (2.1.2) leve o primeiro em $\Gamma_{0}$ e o segundo em $\Sigma_{0}$;

(iv) Todos os pontos da forma $\left(s+k \tau, n_{0}\right), k \in \mathbb{Z}$, com $n_{0}$ fixado, são levados num mesmo ponto da região $\Omega$;

(v) Do item anterior, para cada $s_{0}$ fixado, (2.1.2) é injetora em $\left\{(s, n) \in \mathbb{R}^{2} \mid s \in\right.$ $\left.\left[s_{0}, s_{0}+\tau\right), n \in(-\delta, \delta)\right\}$.

Das propriedades acima, segue que (2.1.2) é um homeomorfismo local e portanto, uma mudança de coordenadas local do sistema (2.0.1).

Seja a mudança de coordenadas (2.1.2). Diferenciando com respeito a $t$, obtemos:

$$
\left\{\begin{array}{l}
\frac{d x}{d t}=\bar{\varphi}_{s}^{\prime} \frac{d s}{d t}+\bar{\varphi}_{n}^{\prime} \frac{d n}{d t}=P(\bar{\varphi}(s, n), \bar{\psi}(s, n)), \\
\frac{d x}{d t}=\bar{\psi}_{s}^{\prime} \frac{d s}{d t}+\bar{\psi}_{n}^{\prime} \frac{d n}{d t}=Q(\bar{\varphi}(s, n), \bar{\psi}(s, n)) .
\end{array}\right.
$$

Pela condição (4), $\triangle(s, n) \neq 0$ na faixa (2.1.1). Portanto, o sistema (2.1.3) tem solução para $\frac{d s}{d t}$ e $\frac{d n}{d t}$ :

$$
\left\{\begin{aligned}
\frac{d s}{d t} & =\frac{P(\bar{\varphi}, \bar{\psi}) \bar{\psi}_{n}^{\prime}-Q(\bar{\varphi}, \bar{\psi}) \bar{\varphi}_{n}^{\prime}}{\triangle(s, n)}, \\
\frac{d n}{d t} & =\frac{Q(\bar{\varphi}, \bar{\psi}) \bar{\varphi}_{s}^{\prime}-P(\bar{\varphi}, \bar{\psi}) \bar{\psi}_{s}^{\prime}}{\triangle(s, n)} .
\end{aligned}\right.
$$


Sabemos que $\bar{\varphi}(s, 0)=\varphi(s), \quad \bar{\psi}(s, 0)=\psi(s)$, e como $x=\varphi(t), \quad y=\psi(t), 0 \leq t<\tau$ é solução do sistema (2.0.1), temos:

$$
\left\{\begin{array}{l}
\frac{\partial \bar{\varphi}(s, 0)}{\partial s}=P(\bar{\varphi}(s, 0), \bar{\psi}(s, 0)), \\
\frac{\partial \bar{\psi}(s, 0)}{\partial s}=Q(\bar{\varphi}(s, 0), \bar{\psi}(s, 0)),
\end{array}\right.
$$

para todo $s, 0 \leq s \leq \tau$. Segue que:

$$
\begin{aligned}
& P(\bar{\varphi}(s, 0), \bar{\psi}(s, 0)) \bar{\psi}_{n}^{\prime}(s, 0)-Q(\bar{\varphi}(s, 0), \bar{\psi}(s, 0)) \bar{\varphi}_{n}^{\prime}(s, 0)= \\
& \quad=\bar{\varphi}_{s}^{\prime}(s, 0) \bar{\psi}_{n}^{\prime}(s, 0)-\bar{\psi}_{s}^{\prime}(s, 0) \bar{\varphi}_{n}^{\prime}(s, 0)=\triangle(s, 0) .
\end{aligned}
$$

Pela condição $\triangle(s, n) \neq 0$, concluímos que $\triangle(s, 0) \neq 0$, para todo $s, 0 \leq s \leq \tau$. Portanto, o lado esquerdo da Eq. (2.1.6) não é nulo para todo $s, 0 \leq s \leq \tau$.

Logo, pela continuidade de todas as funções envolvidas e da compacidade do segmento $0 \leq s \leq \tau$, segue que, para todos os valores suficientemente pequenos de $n$ e para todo $\mathrm{s}$, $0 \leq s \leq \tau$ :

$$
P(\bar{\varphi}(s, n), \bar{\psi}(s, n)) \bar{\psi}_{n}^{\prime}(s, n)-Q(\bar{\varphi}(s, n), \bar{\psi}(s, n)) \bar{\varphi}_{n}^{\prime}(s, n) \neq 0 .
$$

Como as funções $\bar{\varphi}$ e $\bar{\psi}$ e suas derivadas são periódicas em $s$, a relação (2.1.7) é satisfeita para todo $s$ e para valores de $n$ suficientemente pequenos. Podemos assumir que (2.1.7) é satisfeita na faixa (2.1.1). Para tanto, basta escolher $\delta>0$ suficientemente pequeno.

Por (2.1.7), o lado direito da primeira equação em (2.1.4) não se anula na faixa (2.1.1). Portanto, as Eqs. (2.1.4) podem ser substituídas por uma única equação diferencial

$$
\frac{d n}{d s}=\frac{Q(\bar{\varphi}, \bar{\psi}) \bar{\varphi}_{s}^{\prime}-P(\bar{\varphi}, \bar{\psi}) \bar{\psi}_{s}^{\prime}}{P(\bar{\varphi}, \bar{\psi}) \bar{\psi}_{n}^{\prime}-Q(\bar{\varphi}, \bar{\psi}) \bar{\varphi}_{n}^{\prime}}=R(s, n)
$$

obtida pelo quociente da segunda equação de (2.1.4) pela primeira.

A função $R(s, n)$ é definida e contínua na faixa (2.1.1), e continuamente diferenciável com respeito a $n$ em (2.1.1). Assim, ambos Teorema de Existência e Unicidade das 
Equações Diferenciais e o Teorema da Dependência Contínua em Relação às Condições Iniciais - cf. [48, 31], são aplicáveis à Eq. (2.1.8).

Do Teorema de Existência e Unicidade segue que, para todo $s_{0}$, e todo $n_{0},\left|n_{0}\right|<\delta$, existe uma única solução de (2.1.8)

$$
n=f\left(s ; s_{0}, n_{0}\right)
$$

a qual está definida em algum intervalo (maximal) $\left(s_{1}, s_{2}\right)$ contendo o ponto $s_{0}$ e satisfaz a condição inicial $f\left(s_{0} ; s_{0}, n_{0}\right)=n_{0}$.

Por (2.1.2) e (2.1.5), segue que $R(s, 0) \equiv 0$.

Em consequência, $n=0$ é solução da Eq. (2.1.8) e, consequentemente, o eixo $s$ do plano $(s, n)$ é uma solução de (2.1.8).

Todas as soluções da Eq. (2.1.8), situadas na faixa (2.1.1), evidentemente coincidem com as soluções de (2.1.4). Se $s=s(t), \quad n=n(t)$ é uma curva solução $\hat{\gamma}$ de (2.1.4) e $\left(s_{0}, n_{0}\right)$ é um ponto desta curva, a solução $n=f\left(s ; s_{0}, n_{0}\right)$ de $(2.1 .4)$ é uma equação de $\hat{\gamma}$ nas coordenadas $s, n$.

A mudança de coordenadas (2.1.2) leva $\hat{\gamma}$ em $x=\bar{\varphi}(s(t), n(t)), \quad y=\bar{\psi}(s(t), n(t))$, a qual, em virtude da regularidade local de (2.1.2) é uma curva integral do sistema (2.0.1) situada na região $\Omega$, por construção. Seja $\gamma$ esta curva. É claro que toda solução $\gamma$ de (2.0.1) em $\Omega$ é a imagem, em (2.1.2), de pelo menos uma curva integral $\hat{\gamma}$ de (2.1.4) na faixa (2.1.1), ou seja, é a imagem de pelo menos uma curva solução da Eq. (2.1.8).

A Eq. (2.1.9) de $\hat{\gamma}$, no plano $(s, n)$, pode ser tratada como uma equação nas coordenadas $s, n$ de $\gamma$, no plano $(x, y)$. Posteriormente, usaremos este fato em nossa análise da função sucessão.

Seja $l$ o segmento da reta normal a $\gamma_{0}$, contido em $\bar{\Omega}$ e que passa pelo ponto $M_{0}$ da curva $\gamma_{0}$ correspondente a $s=0$. Logo, $l$ é uma seção transversal de (2.0.1).

Do Teorema de Existência e Unicidade e do Teorema da Dependência Contínua em Relação às Condições Iniciais, e também do fato de que $n=0$ é solução de (2.1.8), obtemos os seguintes resultados:

Proposição 2.1.1. Toda solução $n=f\left(s ; s_{0}, n_{0}\right) d a E q$. (2.1.8), para todo $s_{0}, 0 \leq s_{0} \leq \tau$ e $n_{0}$ suficientemente pequeno, está definida para todo $s, 0 \leq s \leq \tau$, e pode ser escrita na forma $n=f\left(s ; 0, n_{0}^{*}\right)$. 
Para o sistema (2.0.1), isto significa, em termos geométricos que, toda solução passando por um ponto numa vizinhança suficientemente pequena de $\gamma_{0}$ cruza a normal $l$ (para $s=0$ ) bem como todas as outras normais a $\gamma_{0}$ no anel $\Omega$, e em seguida, cruza novamente a normal $l$ (para $s=\tau$ ), conforme ilustrado na figura abaixo:
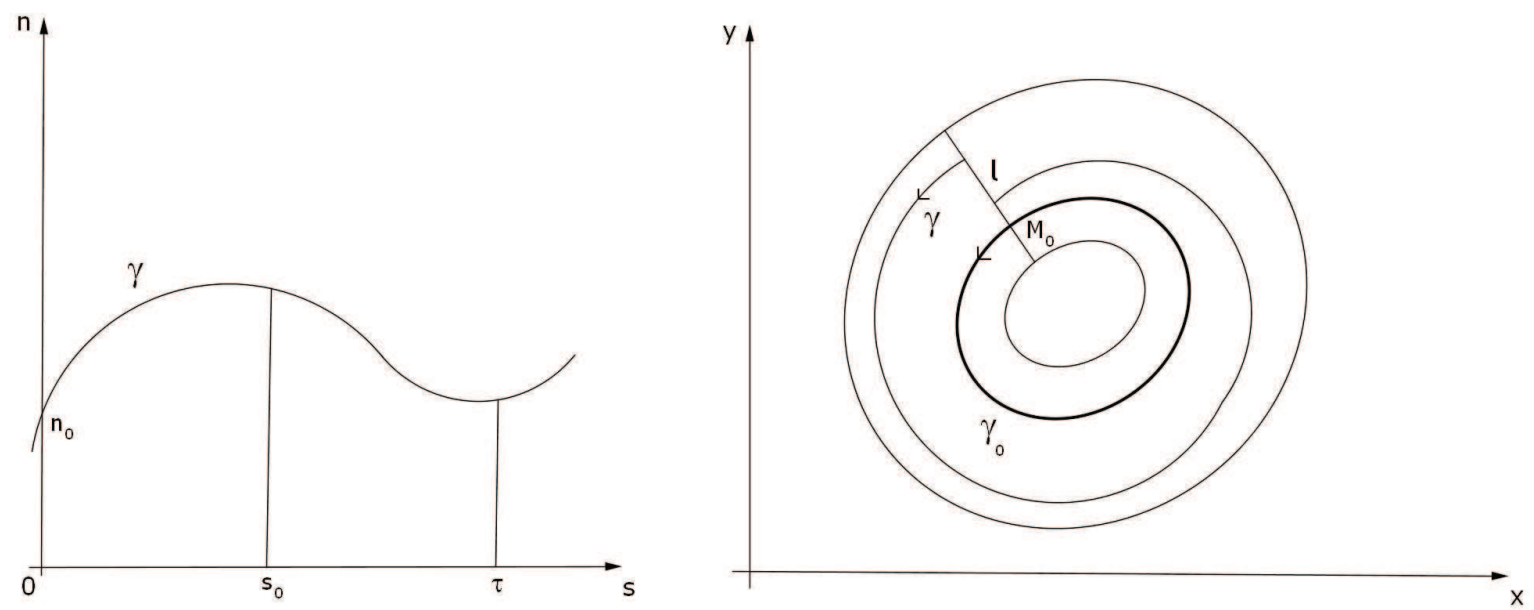

Figura 2.2: Interpretação geométrica da Prop. 2.1.1 para o sistema (2.0.1)

Observe que, para $n=0, \frac{d s}{d t}=1$ (veja (2.1.6) e (2.1.4)), e como tanto o numerador quanto o denominador da expressão para $\frac{d s}{d t}$ em (2.1.4) não revertem seus sinais na faixa (2.1.1), segue que $\frac{d s}{d t}>0$ em (2.1.1), isto é, um incremento em $s$ corresponde a um incremento em $t$, e vice-versa.

Proposição 2.1.2. Para todo $\varepsilon>0$, existe $\eta>0, \eta=\eta(\varepsilon)$, tal que se $\left|n_{0}\right|<\eta$, então

$$
\left|f\left(s ; 0, n_{0}\right)\right|<\varepsilon, \forall s, 0 \leq s \leq \tau
$$

Isso implica que a parte da solução de (2.0.1), situada entre dois pontos de intersecção sucessivos da curva integral e da seção transversal, está completamente contido em $U_{\varepsilon}\left(\gamma_{o}\right)$, se o primeiro ponto de intersecção corresponde a $n_{0}$, o qual é menor que $\eta$ em termos absolutos. 


\section{Aplicação de Poincaré}

A aplicação de Poincaré, também conhecida como aplicação de primeiro retorno é, provavelmente, a ferramenta mais básica para o estudo da estabilidade e bifurcação de órbitas periódicas. Desenvolvida por Poincaré em 1881 - cf. [45], esta técnica oferece diversas vantagens no estudo de EDO's, tais como a redução de dimensão. A seguir, definimos formalmente a aplicação de Poincaré e, em seguida, apresentamos diversas propriedades importantes que serão utilizadas ao longo da dissertação.

Definição 2.1.3. Sejam um campo vetorial $X$ de classe $C^{r}$, com $r \geq 1$ ou $r=\omega$ (analítico), $\gamma$ uma órbita periódica de $X$ passando por um ponto $p$ e $\Sigma$ uma seção transversal de $X$ em $M_{0}$. Definimos a aplicação de Poincaré $\Pi: \Sigma_{0} \rightarrow \Sigma$ como a função de primeiro retorno do fluxo em $\Sigma$, isto é, para cada ponto de $\Sigma$ pertencente a uma órbita suficientemente próxima de $p$, a aplicação de Poincaré fornece o primeiro ponto em que a órbita intercepta $\Sigma$ num tempo positivo.

Vale ressaltar que, na definição acima $\Sigma_{0}$ é escolhido suficientemente pequeno de modo que a aplicação $\Pi$ esteja definida em todos os pontos em $\Sigma_{0}$.

Proposição 2.1.4. Seja $X$ um campo vetorial de classe $C^{r}$, com $r \geq 1$ ou $r=\omega$ (analítico). Então, a aplicação de primeiro retorno $\Pi(x)$ associada a uma órbita fechada $\gamma$ de $X$, numa vizinhança da origem, é um difeomorfismo de classe $C^{r}$ sobre sua imagem $\Sigma_{1}$

Demonstração. Seja a aplicação de primeiro retorno $\Pi: \Sigma_{0} \rightarrow \Sigma$, conforme Definição 2.1.3, com $\Sigma$ uma seção transversal de $X$.

Seja $V$ uma vizinhança da origem, dada pelo Corolário 1.2.4. Como $\varphi\left(\tau_{0}, p\right)=p$, existe uma vizinhança $\Sigma_{0}$ de $p$ em $\Sigma$, tal que $\varphi\left(\tau_{0}, q\right) \in V$ para todo $q \in \Sigma_{0}$. Seja $\xi: V \rightarrow \Sigma$ a aplicação definida no Corolário 1.2.4. Denotemos $\Pi: \Sigma_{0} \rightarrow \Sigma, \Pi(q)=\xi\left(\varphi\left(\tau_{0}, q\right)\right)$.

Uma outra expressão para a Aplicação de Poincaré é $\Pi(q)=\varphi\left(\tau_{0}+\tau\left(\varphi\left(\tau_{0}, q\right)\right), q\right)$, onde $\tau: V \rightarrow \mathbb{R}$ é o tempo $\tau(x)$ que leva a órbita por $x$ em $V$ para interceptar $\Sigma$. Do Teorema da Função Implícita, segue que $\tau$ é de classe $C^{r}$.

Destas expressões, resulta que $\Pi$ é da mesma classe de diferenciabilidade de $X$. 
A aplicação inversa $\Pi^{-1}: \Sigma_{1} \rightarrow \Sigma_{0}$ de $\Pi$ é definida tomando-se o campo $-X$.

Logo, segue que $\Pi$ é um difeomorfismo de classe $C^{r}$.

Proposição 2.1.5. [5] Seja o sistema diferencial analítico planar da forma (2.0.1), e seja $\gamma_{0}$ uma órbita fechada de período $\tau>0$ deste sistema, parametrizada por

$$
\left\{\begin{array}{l}
x=\varphi(t) \\
y=\psi(t)
\end{array}\right.
$$

Então, a derivada da aplicação de Poincaré ao longo de uma seção transversal normal $a \Gamma_{0}=\left\{x \in \mathbb{R}^{2}: x=\gamma_{0}(t)-\gamma_{0}(0), 0 \leq t \leq \tau\right\}$, em $x=0$, é dada por:

$$
\Pi^{\prime}(0)=\exp \left(\int_{0}^{\tau}\left[P_{x}^{\prime}(\varphi(s), \psi(s))+Q_{y}^{\prime}(\varphi(s), \psi(s))\right] d s\right)
$$

Para demonstrarmos esta proposição, usaremos o seguinte lema:

Lema 2.1.6. Sejam o sistema diferencial analítico planar da forma (2.0.1), e $\gamma_{0}$ uma órbita fechada de período $\tau>0$ deste sistema. Se $\delta>0$ é suficientemente pequeno, então nenhum par de segmentos de retas normais passando por dois pontos distintos de $\gamma_{0}$ possui pontos em comum.

A demonstração deste lema pode ser verificada em [5] (Appendix, subsection 4, pp. $470-471)$.

Podemos agora, proceder à demonstração da Proposição 2.1.5:

Demonstração. (Proposição 2.1.5). Conforme discutido na Seção 2.1.1, sejam $l$ seção transversal de (2.0.1) passando pelo ponto $M_{0}$ da curva fechada $\gamma_{0}$, e $f\left(s ; s_{0}, n_{0}\right)$ solução da Eq. (2.1.8).

Como $s$ é crescente em $t$, é claro que a aplicação de primeiro retorno de Poincaré, $\Pi$, na seção transversal $l$ é $f\left(\tau, 0, n_{0}\right)$. De fato, conforme observação feita na Proposição 2.1.1, em termos geométricos, $f\left(\tau, 0, n_{0}\right)$ indica o primeiro ponto em que uma órbita intercepta a seção transversal $l$ em tempo positivo (de fato, em $t=\tau$ ). Portanto,

$$
\Pi\left(n_{0}\right)=f\left(\tau, 0, n_{0}\right) .
$$


Como $n=0$ é uma solução da Eq. (2.1.8), segue que

$$
\Pi(0)=f(\tau ; 0,0) \equiv 0
$$

Por definição, $f\left(s ; 0, n_{0}\right)$ é uma solução da Eq. (2.1.8). Logo,

$$
\frac{d f\left(s ; 0, n_{0}\right)}{d s}=R\left(s, f\left(s ; 0, n_{0}\right)\right)
$$

Diferenciando com respeito a $n_{0}$, obtemos

$$
\frac{\partial}{\partial n_{0}}\left(\frac{d f\left(s ; 0, n_{0}\right)}{d s}\right)=\frac{\partial R\left(s, f\left(s ; 0, n_{0}\right)\right)}{\partial n} \frac{\partial f\left(s ; 0, n_{0}\right)}{\partial n_{0}}
$$

Como $R(s, n)$ é uma função contínua com derivada parcial contínua com respeito a $n$, a derivada mista no primeiro membro da Eq. (2.1.13) é contínua e independe da ordem de diferenciação - cf. [46], Teorema 17, p. 197. Assim, podemos escrever:

$$
\frac{d}{d s}\left(\frac{\partial f\left(s ; 0, n_{0}\right)}{\partial n_{0}}\right)=\frac{\partial R\left(s, f\left(s ; 0, n_{0}\right)\right)}{\partial n} \frac{\partial f\left(s ; 0, n_{0}\right)}{\partial n_{0}}
$$

Por definição, $f\left(0 ; 0, n_{0}\right) \equiv n_{0}$. Portanto,

$$
\left.\frac{\partial f\left(s ; 0, n_{0}\right)}{\partial n_{0}}\right|_{s=0}=1
$$

Tomando $n_{0}=0$ na Eq. (2.1.13) e integrando com a condição inicial (2.1.14), obtemos

$$
\left.\frac{\partial f\left(s ; 0, n_{0}\right)}{\partial n_{0}}\right|_{n_{0}=0}=\exp \left(\int_{0}^{s} \frac{\partial R(s, f(s ; 0,0))}{\partial n} d s\right) .
$$

Por (2.1.12), segue que $f(s ; 0,0)=0$. Assim, o integrando em $(2.1 .15)$ é igual a $\frac{\partial R(s, 0)}{\partial n}$.

Por outro lado, diferenciando (2.1.8) com respeito a $n$ resulta em:

$$
\begin{aligned}
\frac{\partial R(s, n)}{\partial n}= & \frac{-\left[P_{x}^{\prime} \bar{\varphi}_{n}^{\prime}+P_{y}^{\prime} \bar{\psi}_{n}^{\prime}\right] \bar{\psi}_{s}^{\prime}+\left[Q_{x}^{\prime} \bar{\varphi}_{n}^{\prime}+Q_{y}^{\prime} \bar{\psi}_{n}^{\prime}\right] \bar{\varphi}_{s}^{\prime}-P \bar{\psi}_{s n}^{\prime \prime}+Q \bar{\varphi}_{s n}^{\prime \prime}}{P \bar{\psi}_{n}^{\prime}-Q \bar{\varphi}_{n}^{\prime}}+ \\
& +\frac{P \bar{\psi}_{s}^{\prime}-Q \bar{\varphi}_{s}^{\prime}}{\left(P \bar{\psi}_{n}^{\prime}-Q \bar{\varphi}_{n}^{\prime}\right)^{2}} \frac{\partial}{\partial n}\left(P \bar{\psi}_{n}^{\prime}-Q \bar{\varphi}_{n}^{\prime}\right)
\end{aligned}
$$


onde $P=P(\bar{\varphi}, \bar{\psi}), P_{x}^{\prime}=P_{x}^{\prime}(\bar{\varphi}, \bar{\psi})$, e assim por diante.

De (2.1.5) segue que, para $n=0, P(\bar{\varphi}, \bar{\psi}) \bar{\psi}_{s}^{\prime}-Q(\bar{\varphi}, \bar{\psi}) \bar{\varphi}_{s}^{\prime} \equiv 0$. Consequentemente, para $n=0$, apenas a primeira fração permanece no lado direito da Eq. (2.1.16). Temos $\frac{\partial \varphi}{\partial s}=P(\varphi(s), \psi(s)), \quad \frac{\partial \psi}{\partial s}=Q(\varphi(s), \psi(s))$.

Diferenciando com respeito a $s$, obtemos

$$
\begin{aligned}
& \varphi^{\prime \prime}(s)=P_{x}^{\prime}(\varphi(s), \psi(s)) \varphi^{\prime}(s)+P_{y}^{\prime}(\varphi(s), \psi(s)) \psi^{\prime}(s), \\
& \psi^{\prime \prime}(s)=Q_{x}^{\prime}(\varphi(s), \psi(s)) \varphi^{\prime}(s)+Q_{y}^{\prime}(\varphi(s), \psi(s)) \psi^{\prime}(s) .
\end{aligned}
$$

Logo,

$$
\begin{aligned}
& P_{y}^{\prime}(\varphi(s), \psi(s)) \psi^{\prime}(s)=\varphi^{\prime \prime}(s)-P_{x}^{\prime}(\varphi(s), \psi(s)) \varphi^{\prime}(s), \\
& Q_{y}^{\prime}(\varphi(s), \psi(s)) \psi^{\prime}(s)=\psi^{\prime \prime}(s)-Q_{x}^{\prime}(\varphi(s), \psi(s)) \varphi^{\prime}(s) .
\end{aligned}
$$

Tomando $n=0$ em (2.1.16) e usando (2.1.2), (2.1.5), e (2.1.17), obtemos após algumas manipulações algébricas simples:

$$
\begin{aligned}
\frac{\partial R(s, 0)}{\partial n} & =P_{x}^{\prime}(\varphi(s), \psi(s))+Q_{y}^{\prime}(\varphi(s), \psi(s))-\frac{2\left[\varphi^{\prime}(s) \varphi^{\prime \prime}(s)+\psi^{\prime}(s) \psi^{\prime \prime}(s)\right]}{\left[\varphi^{\prime}(s)\right]^{2}+\left[\psi^{\prime}(s)\right]^{2}} \\
& =P_{x}^{\prime}(\varphi(s), \psi(s))+Q_{y}^{\prime}(\varphi(s), \psi(s))-\left[\ln \left(\left[\varphi^{\prime}(s)\right]^{2}+\left[\psi^{\prime}(s)\right]^{2}\right)\right]^{\prime}
\end{aligned}
$$

O primeiro membro desta última relação é o integrando de (2.1.15). Portanto,

$$
\begin{aligned}
\left.\frac{\partial f\left(s ; 0, n_{0}\right)}{\partial n_{0}}\right|_{n_{0}=0} & =\exp \left(\int_{0}^{s}\left\{\left[P_{x}^{\prime}+Q_{y}^{\prime}\right]-\left[\ln \left(\left[\varphi^{\prime}(s)\right]^{2}+\left[\psi^{\prime}(s)\right]^{2}\right)\right]^{\prime}\right\} d s\right) \\
& =\frac{\left[\varphi^{\prime}(0)\right]^{2}+\left[\psi^{\prime}(0)\right]^{2}}{\left[\varphi^{\prime}(s)\right]^{2}+\left[\psi^{\prime}(s)\right]^{2}} \exp \left(\int_{0}^{s}\left[P_{x}^{\prime}(\varphi(s), \psi(s))+Q_{y}^{\prime}(\varphi(s), \psi(s))\right] d s\right)
\end{aligned}
$$

De (2.1.11) e (2.1.18), tomando $s=\tau$ e lembrando que as funções $\varphi$ e $\psi$ e suas respectivas derivadas são periódicas, obtemos a expressão para $\Pi^{\prime}(0)$ :

$$
\Pi^{\prime}(0)=\left.\frac{\partial f\left(\tau ; 0, n_{0}\right)}{\partial n_{0}}\right|_{n_{0}=0}=\exp \left(\int_{0}^{\tau}\left[P_{x}^{\prime}(\varphi(s), \psi(s))+Q_{y}^{\prime}(\varphi(s), \psi(s))\right] d s\right)
$$


Nosso próximo passo é definir a função sucessão a partir da aplicação de Poincaré e estudar suas propriedades.

\section{Função sucessão}

Definição 2.1.7. Seja $\Pi(x)$ a aplicação de primeiro retorno para uma órbita fechada $\gamma$ de um sistema diferencial analítico (2.0.1). Definimos a função sucessão como sendo

$$
d(x)=\Pi(x)-x
$$

Apresentamos a seguir, algumas propriedades da função sucessão, relativas a sistemas diferenciais analíticos da forma (1.2.1), isto é, sistemas em que a origem é um ponto singular isolado não degenerado, e os autovalores de sua parte linear na origem são complexos conjugados $\lambda_{1,2}=\alpha \pm i \beta, \beta \neq 0$ (vide Proposição 1.2.8).

Seja um sistema diferencial analítico da forma (1.2.1). Efetuando-se mudança de coordenadas polares

$$
\left\{\begin{array}{l}
x=\rho \cos (\theta), \\
y=\rho \operatorname{sen}(\theta)
\end{array}\right.
$$

segue que $\dot{x}=\dot{\rho} \cos (\theta)-\dot{\theta} \rho \operatorname{sen}(\theta)$ e $\dot{y}=\dot{\rho} \operatorname{sen}(\theta)+\dot{\theta} \rho \cos (\theta)$, onde $\dot{x}=\frac{d x}{d t}$, valendo a mesma notação para as demais variáveis. Assim,

$$
\begin{aligned}
\dot{\rho}= & \dot{x} \cos (\theta)+\dot{y} \operatorname{sen}(\theta) \\
& \stackrel{(2.1 .19)}{=} \cos (\theta)(\alpha \rho \cos (\theta)-\beta \rho \operatorname{sen}(\theta)+F(\rho \cos (\theta), \rho \operatorname{sen}(\theta))) \\
& +\operatorname{sen}(\theta)(\beta \rho \cos (\theta)+\alpha \rho \operatorname{sen}(\theta)+G(\rho \cos (\theta), \rho \operatorname{sen}(\theta))) .
\end{aligned}
$$

Denotando $F(\rho \cos (\theta), \rho \operatorname{sen}(\theta)):=F(\rho, \theta)$ e $G(\rho \cos (\theta), \rho \operatorname{sen}(\theta)):=G(\rho, \theta)$ e efetuando cálculos simples, obtemos:

$$
\dot{\rho}=\alpha \rho+F(\rho, \theta) \cos (\theta)+G(\rho, \theta) \operatorname{sen}(\theta):=\Phi(\rho, \theta)
$$


Analogamente,

$$
\begin{aligned}
\rho \dot{\theta}= & -\dot{x} \operatorname{sen}(\theta)+\dot{y} \cos (\theta) \\
= & -\operatorname{sen}(\theta)(\alpha \rho \cos (\theta)-\beta \rho \operatorname{sen}(\theta)+F(\rho, \theta))+ \\
& +\cos (\theta)(\beta \rho \cos (\theta)+\alpha \rho \operatorname{sen}(\theta)+G(\rho, \theta)) .
\end{aligned}
$$

Logo,

$$
\dot{\theta}=\beta+\frac{\cos (\theta) G(\rho, \theta)}{\rho}-\frac{\operatorname{sen}(\theta) F(\rho, \theta)}{\rho}:=\beta+\Psi(\rho, \theta)
$$

Obtemos, deste modo, o seguinte sistema diferencial analítico:

$$
\left\{\begin{array}{l}
\frac{d \rho}{d t}=\Phi(\rho, \theta), \\
\frac{d \theta}{d t}=\beta+\Psi(\rho, \theta)
\end{array}\right.
$$

onde

$$
\begin{aligned}
& \Phi(\rho, \theta)=\alpha \rho+F(\rho \cos (\theta), \rho \operatorname{sen}(\theta)) \cos (\theta)+G(\rho \cos (\theta), \rho \operatorname{sen}(\theta)) \operatorname{sen}(\theta), \\
& \Psi(\rho, \theta)=\frac{G(\rho \cos (\theta), \rho \operatorname{sen}(\theta))}{\rho} \cos (\theta)-\frac{F(\rho \cos (\theta), \rho \operatorname{sen}(\theta))}{\rho} \operatorname{sen}(\theta) .
\end{aligned}
$$

Assumiremos que $\Psi(0, \theta) \equiv 0,-\infty<\theta<+\infty$. Esta condição garante a continuidade da função $\Psi$.

Usando argumentos semelhantes aos empregados na obtenção da Eq. (2.1.8) (vide seção 2.1.1), o sistema (2.1.20) pode ser reduzido para:

$$
\frac{d \rho}{d \theta}=R(\rho, \theta)=\frac{\Phi(\rho, \theta)}{\beta+\Psi(\rho, \theta)}
$$

O sistema (2.1.20) e a Eq. (2.1.21) são considerados na faixa $-\rho^{*}<\rho<+\rho^{*}$ do plano $(\rho, \theta)$, onde $\rho^{*}$ é um inteiro positivo suficientemente pequeno.

Efetuando-se cálculos simples, constatamos que a função $R(\rho, \theta)$ tem derivada parcial contínua com respeito a $\rho$ em toda a faixa definida acima, e

$$
\left.\frac{\partial R(\rho, \theta)}{\partial \rho}\right|_{\rho=0}=\frac{\alpha}{\beta},
$$


para todo valor de $\theta$.

Podemos então, aplicar os Teorema de Existência e Unicidade e Teorema da Dependência Contínua em Relação às Condições Iniciais à Eq. (2.1.21), na faixa $-\rho^{*}<\rho<+\rho^{*}$. Logo, para quaisquer $\theta_{0}$ e $\rho_{0},\left|\rho_{0}\right|<\rho^{*}$, existe uma única solução de (2.1.21):

$$
\rho=f\left(\theta, \theta_{0}, \rho\right)
$$

satisfazendo a condição

$$
f\left(\theta_{0}, \theta_{0}, \rho_{0}\right) \equiv \rho_{0} .
$$

Convém salientar que, a solução acima está definida em algum intervalo (maximal) $\left(\theta_{1}, \theta_{2}\right)$ contendo o ponto $\theta_{0}$. Além disso,

$$
f\left(\theta ; \theta_{0}, 0\right) \equiv 0
$$

e assim, $\rho \equiv 0$ é uma solução de (2.1.21).

Por definição, $f\left(\theta ; 0, \rho_{0}\right)$ satisfaz $\frac{d f\left(\theta ; 0, \rho_{0}\right)}{d \theta}=R\left(f\left(\theta ; 0, \rho_{0}\right), \theta\right)$.

Agora, aplicando argumentos semelhantes àqueles empregados na demonstração da Proposição 2.1.5, obtemos:

$$
\frac{\partial f(\theta ; 0,0))}{\partial \rho_{0}}=\exp \left(2 \pi \frac{\alpha}{\beta}\right)
$$

É importante destacar que a Eq. (2.1.21) não é afetada pela substituição simultânea de $\rho$ por $-\rho$ e de $\theta$ por $\theta+\pi$. Mais precisamente, se $\rho=\rho(\theta)$ é uma solução de (2.1.21), e se $\rho^{*}=-\rho$ e $\theta^{*}=\theta+\pi$, então $\frac{d \rho^{*}}{d \theta^{*}}=R\left(\rho^{*}, \theta^{*}\right)$. De fato,

$$
\frac{d \rho^{*}}{d \theta^{*}}=-\frac{d \rho}{d(\theta+\pi)}=-\frac{d \rho}{d \theta}=-R(\rho, \theta)=R\left(\rho^{*}, \theta^{*}\right)
$$

A última igualdade segue diretamente de (2.1.20) e (2.1.21). Logo, se $\rho=\rho(\theta)$ é uma solução da Eq. (2.1.21), então

$$
\frac{d(-\rho)}{d(\theta+\pi)}=R(-\rho, \theta+\pi) \equiv-R(\rho, \theta)
$$


De maneira análoga à seção 2.1.1, podemos definir a aplicação de primeiro retorno sobre o raio $\theta=\theta_{0}$, como

$$
\Pi_{\theta_{0}}\left(\rho_{0}\right)=f_{\theta_{0}}\left(\rho_{0}\right)=f\left(\theta_{0}+2 \pi ; \theta_{0}, \rho_{0}\right)
$$

Portanto, a função sucessão é dada por

$$
d_{\theta_{0}}\left(\rho_{0}\right)=f_{\theta_{0}}\left(\rho_{0}\right)-\rho_{0}
$$

Em muitos casos, não há prejuízo em tomarmos $\theta_{0}=0$ (bastando para isso, fazer uma rotação de $\theta_{0}$ no sistema). Então, em geral, podemos tomar a aplicação de Poincaré e a função sucessão como sendo, respectivamente

$$
\begin{aligned}
& \Pi\left(\rho_{0}\right)=f\left(2 \pi ; 0, \rho_{0}\right) \\
& d\left(\rho_{0}\right)=\Pi\left(\rho_{0}\right)-\rho_{0}=f\left(2 \pi ; 0, \rho_{0}\right)-\rho_{0} .
\end{aligned}
$$

Proposição 2.1.8. Sejam um sistema diferencial analítico da forma (2.1.20) (expressão de (1.2.1) em coordenadas polares), tal que a origem seja um ponto singular isolado não degenerado, $\Pi\left(\rho_{0}\right)$ a função de primeiro retorno deste sistema definida numa seção transversal $\Sigma$, e d $\left(\rho_{0}\right)$ a função sucessão. Então a derivada da função sucessão ao longo da seção transversal, na origem, vale $d^{\prime}(0)=\exp \left(2 \pi \frac{\alpha}{\beta}\right)-1$.

Demonstração. As expressões da aplicação de primeiro retorno e da função sucessão são dadas por (2.1.29). Derivando essas expressões em relação a $\rho_{0}$ obtemos:

$$
\begin{aligned}
\Pi^{\prime}\left(\rho_{0}\right) & =\frac{\partial f\left(2 \pi ; 0, \rho_{0}\right)}{\partial \rho_{0}} \\
d^{\prime}\left(\rho_{0}\right) & =\frac{\partial f\left(2 \pi ; 0, \rho_{0}\right)}{\partial \rho_{0}}-1 .
\end{aligned}
$$

Para $\rho_{0}=0$, e usando (2.1.25), segue que

$$
d^{\prime}(0)=\frac{\partial f(2 \pi ; 0,0)}{\partial \rho_{0}}-1=\exp \left(2 \pi \frac{\alpha}{\beta}\right)-1
$$


Desta proposição, segue imediatamente que a expressão da derivada da aplicação de primeiro retorno em zero vale $\Pi^{\prime}(0)=\exp \left(2 \pi \frac{\alpha}{\beta}\right)$. Além disso, para sistemas diferenciais analíticos da forma (1.2.1), com autovalores puramente imaginários, isto é, com $\alpha=$ $0, \beta \neq 0$, segue que as expressões das derivadas da aplicação de primeiro retorno e da função sucessão, em zero, se reduzem respectivamente a $\Pi^{\prime}(0)=1$, e $d^{\prime}(0)=0$.

Proposição 2.1.9. Sejam um sistema diferencial analítico da forma (2.1.20) (expressão de (1.2.1) em coordenadas polares), $d_{\theta_{0}}\left(\rho_{0}\right)$ a função sucessão sobre o raio $\theta=\theta_{0}$, e $\rho=\rho(\theta)$ uma solução de (2.1.21). Então

$$
d_{\theta_{0}}(-\rho)=-d_{\theta_{0}+\pi}(\rho)=-f\left(\theta_{0}+3 \pi ; \theta_{0}+\pi, \rho\right)+\rho .
$$

Demonstração. Por (2.1.28), segue que

$$
d_{\theta_{0}}\left(-\rho_{0}\right)=f\left(\theta_{0}+2 \pi ; \theta_{0},-\rho_{0}\right)-\left(-\rho_{0}\right)
$$

onde $\rho=f\left(\theta ; \theta_{0},-\rho_{0}\right)$ é solução da Eq. (2.1.21) para as condições iniciais $\theta=\theta_{0}, \rho=-\rho_{0}$.

Considere a solução da Eq. (2.1.26) para condições iniciais $\rho^{*}=-\rho=\rho_{0}, \theta^{*}=\theta+\pi=$ $\theta_{0}+\pi$. Esta solução tem a forma:

$$
\rho^{*}=-\rho=f\left(\theta^{*} ; \theta_{0}+\pi, \rho_{0}\right)=f\left(\theta+\pi ; \theta_{0}+\pi, \rho_{0}\right) .
$$

Portanto, para as condições iniciais particulares escolhidas $\rho=-f\left(\theta+\pi ; \theta_{0}+\pi, \rho_{0}\right)$. Mas a condição $\rho^{*}=\rho_{0}$, para $\theta^{*}=\theta_{0}+\pi$ é equivalente à condição $\rho=-\rho_{0}$ para $\theta=\theta_{0}$. Em virtude do Teorema de Existência e Unicidade,

$$
-f\left(\theta+\pi ; \theta_{0}+\pi, \rho_{0}\right) \equiv f\left(\theta ; \theta_{0},-\rho_{0}\right) .
$$

Por (2.1.30), (2.1.31), (2.1.27) e (2.1.28), segue que

$$
\begin{aligned}
d_{\theta_{0}}\left(-\rho_{0}\right) & =f\left(\theta_{0}+2 \pi ; \theta_{0},-\rho_{0}\right)-\left(-\rho_{0}\right)=-f\left(\theta_{0}+3 \pi ; \theta_{0}+\pi, \rho_{0}\right)+\rho_{0} \\
& =-\left[f_{\theta_{0}+\pi}\left(\rho_{0}\right)-\rho_{0}\right]=-d_{\theta_{0}+\pi}\left(\rho_{0}\right) .
\end{aligned}
$$

Substituindo $\rho$ por $\rho_{0}$, obtemos a expressão desejada. 
Proposição 2.1.10. Nas condições da proposição anterior, se existir $r_{1}>0$ tal que, para todo $\rho, 0<|\rho| \leq r_{1}, d_{\theta_{0}}(\rho)>0\left(d_{\theta_{0}}(\rho)<0\right)$, então também existe $r_{2}>0$ tal que para todo $\rho, 0<|\rho| \leq r_{2}, d_{\theta_{0}}(-\rho)<0\left(d_{\theta_{0}}(-\rho)>0\right.$, respectivamente). Portanto, para todo $\rho, 0<|\rho| \leq r=\min \left(r_{1}, r_{2}\right)$, vale $d_{\theta_{0}}(\rho) d_{\theta_{0}}(-\rho)<0$.

Demonstração. Faremos a demonstração para o caso em que para todo $\rho, 0<\rho \leq r_{1}$, $d_{\theta_{0}}(\rho)>0$. O caso $d_{\theta_{0}}(\rho)<0$ é análogo.

Seja $\Sigma$ uma seção transversal local parametrizada por $(\rho, \theta), 0<\rho \leq r_{1}, \theta=\theta_{0}$. Então, todas as órbitas do sistema (1.2.1), passando pelos pontos da forma $\left(\theta_{0}, \rho\right)$, não são fechadas. De fato, estas órbitas são espirais que divergem da origem para $t \rightarrow \infty$. Logo, todas essas órbitas cruzam os raios $\theta=$ constante, em particular, o raio $\theta=\theta_{0}+\pi$.

A aplicação de primeiro retorno $\Pi_{\theta_{0}+\pi}(\rho)=f\left(\theta_{0}+2 \pi ; \theta_{0}+\pi, \rho\right)$ e a função sucessão $d_{\theta_{0}+\pi}(\rho)=\Pi_{\theta_{0}+\pi}(\rho)-\rho$ estão a priori definidas para todo $\rho, 0<|\rho| \leq r_{2}$, onde $r_{2}$ é algum inteiro positivo suficientemente pequeno tal que $\Pi$ e $d_{\theta_{0}+\pi}$ estejam bem definidas em $\Sigma$.

Como por hipótese, $d_{\theta_{0}}(\rho)>0$, segue que para todo $\rho, 0<|\rho| \leq r_{2}$, temos $d_{\theta_{0}+\pi}(\rho)>$ 0. Então, pela Proposição 2.1.9, a função $d_{\theta_{0}}(-\rho)=-d_{\theta_{0}+\pi}(\rho)$ está definida para todo $\rho, 0<|\rho| \leq r_{2}$ e é negativa.

Desta forma, para todo $\rho, 0<|\rho| \leq r=\min \left(r_{1}, r_{2}\right)$, temos $d_{\theta_{0}}(\rho) d_{\theta_{0}}(-\rho)<0$.

A figura a seguir fornece uma interpretação geométrica do argumento.

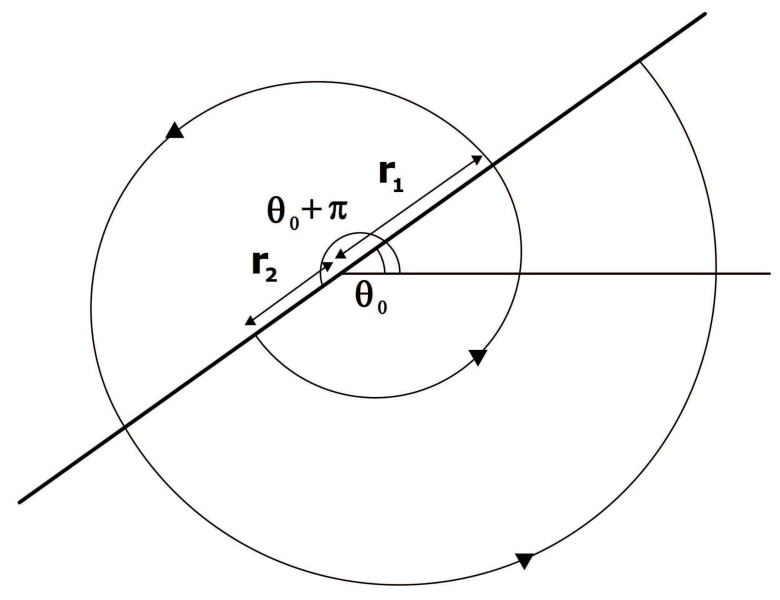

Figura 2.3: Interpretação geométrica da Prop. 2.1.10 
Proposição 2.1.11. Nas condições da proposição anterior, se existir $k$ tal que

$$
d^{\prime}(0)=d^{\prime \prime}(0)=\ldots=d^{k-1}=0, d^{k} \neq 0
$$

então $k$ é um número ímpar.

Demonstração. Por (2.1.20) e (2.1.21), concluímos que $\rho \equiv 0$ é uma solução do sistema (2.1.21) e portanto, $f(0)=d(0)=0$. Como o sistema (2.1.21) é analítico, pela Proposição 2.1.4 segue que $\Pi$ e $d$ são analíticas numa vizinhança da origem. Usando estes fatos e a hipótese (2.1.32), segue que a expansão em série de Taylor da função sucessão é dada por

$$
d\left(\rho_{0}\right)=\frac{d^{(k)}\left(\rho_{0}\right)}{k !} \rho_{0}^{k}+o\left(\left\|\rho_{0}\right\|^{k+1}\right)
$$

Logo, se $k$ for par, $d\left(\rho_{0}\right)$ tem o mesmo sinal para todo valor de $\rho_{0}$ suficientemente pequeno, coincidindo com o sinal de $\frac{d^{(k)}\left(\rho_{0}\right)}{k !}$. Entretanto, isto contradiz a Proposição 2.1.10. Assim, $k$ deve ser ímpar.

\subsubsection{Constantes de Lyapunov}

Nesta seção, apresentaremos as constantes de Lyapunov, uma das ferramentas fundamentais no estudo do problema do centro-foco em sistemas diferenciais com singularidades não degeneradas. Consideramos sistemas da forma (1.2.1), com $\alpha=0$, conforme Proposição 1.2.8. Observamos que, após mudança de variáveis e reescalonamento de tempo convenientes, tais sistemas assumem a forma (1.2.5).

Seja um sistema diferencial analítico da forma (1.2.5), tendo a origem como singularidade não degenerada (a origem pode ser tomada como ponto singular sem perda de generalidade, conforme mostrado no Lema 1.2.7), e sua respectiva Aplicação de Poincaré, dada por:

$$
\Pi: U \cap[0, \alpha) \longrightarrow(0, \beta)
$$

onde os intervalos $[0, \alpha)$ e $(0, \beta)$ são subconjuntos do semieixo $O X^{+}$.

Para qualquer ponto da forma $(x, 0) \in \mathbb{R}^{2}, 0<x<\alpha$, a Aplicação de Poincaré fornece o primeiro retorno $(\Pi(x), 0)$ ao semieixo $O X^{+}$, da órbita que passa por $(x, 0), \Pi(0)=0$. 
Pela Proposição 2.1.4, a Aplicação de Poincaré do sistema (1.2.1) é analítica numa vizinhança $U$ da origem. Além disso, pela Proposição 2.1.8, $\Pi^{\prime}(0)=1$.

Então, a expansão em série de Taylor da Aplicação de Poincaré, na vizinhança $U$ da origem, é dada por:

$$
\Pi(x)=x+\sum_{n \geq 2} \frac{\Pi^{n}(0)}{n !} x^{n} .
$$

A Proposição 2.1.11 assegura que o primeiro termo não-nulo em (2.1.33), caso exista, tem grau ímpar.

Observe que, se $\Pi(x)=x$, isto é, $\frac{\Pi^{n}(0)}{n !} \equiv 0, \forall n \geq 2$, então a órbita passando por $(x, 0)$ é periódica. Como $x$ é um valor qualquer tomado em $U \cap[0, \alpha)$, com $[0, \alpha)$ subconjunto do semieixo $O X^{+}$, segue que todas as órbitas passando por pontos em $U \cap$ $[0, \alpha)$ são periódicas. Ou seja, a origem é um centro. Por outro lado, se existir $k \in \mathbb{N}^{*}$, tal que $\frac{\Pi^{2 k+1}(0)}{2 k+1 !} \neq 0$, então a aplicação de primeiro retorno não é igual à função identidade e neste caso, temos um foco. Estas propriedades motivam a seguinte definição:

Definição 2.1.12. [38] O número real

$$
V_{2 k+1}=\frac{\Pi^{2 k+1}(0)}{(2 k+1) !}
$$

para $k \in \mathbb{N}^{*}$, é denominado a k-ésima constante de Lyapunov.

Segue das considerações acima que, se para algum $k \geq 1$ temos $V_{3}=V_{5}=\ldots=$ $V_{2 k-1}=0$ e $V_{2 k+1} \neq 0$, então a origem é um foco. Se por outro lado, todas as constantes de Lyapunov forem nulas, então a função de primeiro retorno é igual à função identidade e assim, a origem é um centro. Para maiores detalhes, consultar [7].

Para sistemas representados por equações da forma (1.2.5), é possível mostrar que as constantes de Lyapunov são polinômios nos coeficientes de $F$ e $G$ - cf. [5]. Desta forma, podemos obter as condições necessárias para que a origem seja um centro, impondo que as constantes de Lyapunov sejam nulas.

Vale ressaltar que, no caso em que as funções $F$ e $G$ (Eq. (1.2.5)) são polinômios de grau $k$ fixado, pelo Teorema da Base de Hilbert é necessário calcular apenas um número finito de constantes de Lyapunov para obter a caracterização de uma família de sistemas diferenciais. Entretanto, existem obstruções significativas nesta abordagem: 
- Obstruções computacionais: os algoritmos desenvolvidos para computar as constantes de Lyapunov precisam manipular extensas e complexas expressões algébricas, especialmente para famílias de sistemas com uma grande quantidade de parâmetros. Mesmo o uso de sofisticadas técnicas, como as ferramentas de álgebra simbólica computacional, ainda não são capazes de lidar com a manipulação algébrica exigida de maneira satisfatória. Para ilustrar o tipo e grau das dificuldades encontradas, considere que para sistemas analíticos genéricos do tipo (1.2.5), $V_{7}$ é um polinômio com 526 monômios nos coeficientes de $F$ e $G$, e $V_{9}$ tem aproximadamente 3800 monômios;

- Obstruções de suficiência: o Teorema da Base de Hilbert, embora estabeleça a existência de uma quantidade finita de constantes Lyapunov a serem calculadas para caracterização de um sistema ou família de sistemas da forma (1.2.5), não explicita que quantidade é essa. Assim, não temos ideia, na prática, de quantas constantes de Lyapunov precisamos calcular para decidir se a singularidade é um foco ou um centro.

Para ilustrar a complexidade da computação simbólica envolvida, chamamos a atenção para o fato de que, enquanto uma forma geral para a primeira e segunda constantes de Lyapunov foram obtidas para sistemas analíticos reais planares já nos anos 40-50 do século passado - cf. [10], as formas gerais da terceira e quarta constantes de Lyapunov foram computadas apenas em 2008 - cf. [34], e suas expressões ocupam cerca de 4 e 45 páginas, respectivamente.

\subsubsection{Algoritmo de Lyapunov}

Descreveremos agora, um método computacionalmente mais eficiente - cf. [10, 12]. Este processo, desenvolvido por Lyapunov, é puramente algébrico. A ideia é construir recursivamente funções de Lyapunov que podem ser utilizadas para determinar a estabilidade do ponto singular.

Inicialmente, apresentamos algumas definições importantes, que serão usadas ao longo desta seção.

Definição 2.1.13 (Estabilidade de Lyapunov). Conside um sistema $\dot{x}=f(t, x)$, onde $f: U \rightarrow \mathbb{R}^{n}$ é contínua e tal que $U \subset \mathbb{R} \times \mathbb{R}^{n}$ é um aberto. Seja $\varphi(t)$ uma órbita deste 
sistema, definida para todo $t \geq 0$. Então $\varphi(t)$ é estável se $\forall \epsilon>0, \exists \delta(\epsilon)>0$ tal que se $\psi(t)$ é uma outra solução do sistema e $|\psi(0)-\varphi(0)|<\delta$, temos $\psi(t)$ definida para todo $t \geq 0 e|\psi(t)-\varphi(t)|<\epsilon, \forall t \geq 0$.

Definição 2.1.14 (Estabilidade assintótica). Dizemos que $\varphi(t)$ é assintoticamente estável se for estável por Liapunov e, além disso, existir um $\bar{\delta}$ tal que se $|\psi(0)-\varphi(0)|<\bar{\delta} \Rightarrow$ $\lim _{t \rightarrow \infty}|\psi(t)-\varphi(t)|=0$.

Considere $\mathcal{H}^{n}$ o espaço vetorial dos polinômios homogêneos de grau $n$ nas variáveis $x$ e $y$.

Seja o sistema:

$$
\left\{\begin{array}{l}
\dot{x}=-y+P(x, y), \\
\dot{y}=x+Q(x, y) .
\end{array}\right.
$$

onde

$$
\left\{\begin{array}{l}
P(x, y)=\sum_{j=2}^{+\infty} P_{j}(x, y), \\
Q(x, y)=\sum_{j=2}^{+\infty} Q_{j}(x, y) .
\end{array}\right.
$$

com $P_{j}, Q_{j} \in \mathcal{H}^{j}, j \geq 2$. Observe que a origem é uma singularidade isolada de (2.1.35).

Além disso, considere uma função $V$ representada formalmente por:

$$
V(x, y)=\frac{1}{2}\left(x^{2}+y^{2}\right)+\sum_{j=3}^{+\infty} V_{j}(x, y)
$$

onde cada $V_{j} \in \mathcal{H}^{j}, j \geq 3$ e $X(x, y)=(-y+P(x, y), x+Q(x, y))$ é o campo vetorial associado ao sistema (2.1.35).

Para determinarmos a estabilidade da origem, analisaremos a derivada de $V$ na direção do campo vetorial $X$, conforme definição abaixo:

$$
\mathcal{L}_{X} V(x, y):=\dot{V}(x, y)=\nabla V(x, y) \cdot X(x, y) .
$$

Se $V(x, y)>0$ e $\dot{V}(x, y)=\leq 0$ em alguma vizinhança da origem, então $V$ é chamada de função de Lyapunov para o sistema (2.1.35), em $(x, y)=(0,0)$ - cf. [44].

O teorema a seguir trata da estabilidade do ponto singular para o sistema (2.1.35). 
Teorema 2.1.15. Se $V$ é uma função de Lyapunov em $(x, y)=(0,0)$ para o sistema (2.1.35) em alguma vizinhança da origem, então o ponto de equilíbrio na origem é estável. Se, além disso, $\dot{V}<0$, então a singularidade na origem é assintoticamente estável.

A demonstração deste teorema pode ser encontrada em [16].

A ideia de Lyapunov foi construir recursivamente a função $V$ por meio de sucessivas aplicações do Teorema 2.1.15 ao sistema (2.1.35). Mostraremos que a construção da função $V$ produz os coeficientes da expansão de Taylor da função sucessão e consequentemente, fornece as constantes de Lyapunov para o sistema (2.1.35).

Seja o campo vetorial planar $R(x, y)=(-y, x)$ e observe que, se $V$ é uma função definida no plano, então a derivada de $V$ na direção do campo vetorial $R$ pode ser vista como a ação de um operador diferencial linear em V, definido por:

$$
\mathcal{L}_{R}:=-y \frac{\partial}{\partial x}+x \frac{\partial}{\partial y}
$$

Em particular, $\mathcal{L}_{R}$ atua no espaço vetorial $\mathcal{H}^{n}$ da seguinte forma:

$$
\left(\mathcal{L}_{R} V\right)(x, y)=-y V_{x}(x, y)+x V_{y}(x, y)
$$

Aplicando a definição dada pela Eq. (2.1.37), obtemos:

$$
\begin{aligned}
\mathcal{L}_{X} V(x, y)= & \left(-y+\sum_{j=2}^{+\infty} P_{j}(x, y)\right)\left(x+\sum_{j=3}^{+\infty} V_{j x}(x, y)\right) \\
& +\left(x+\sum_{j=2}^{+\infty} Q_{j}(x, y)\right)\left(y+\sum_{j=3}^{+\infty} V_{j y}(x, y)\right)
\end{aligned}
$$

onde os subscritos $x$ e $y$ denotam as derivadas parciais em relação às respectivas variáveis. Além disso, se coletarmos os termos do segundo membro desta identidade, de acordo com seus graus, temos:

$$
\mathcal{L}_{X} V(x, y)=x P_{2}(x, y)+y Q_{2}(x, y)+\left(\mathcal{L}_{R} V_{3}\right)(x, y)+O\left(\left\|x^{2}+y^{2}\right\|^{2}\right)
$$

$\operatorname{com} x P_{2}(x, y)+y Q_{2}(x, y) \in \mathcal{H}^{3}$.

As provas dos próximos lemas e teoremas encontram-se em [16]. 
Lema 2.1.16. Se n é um inteiro impar, então $\mathcal{L}_{R}: \mathcal{H}^{n} \rightarrow \mathcal{H}^{n}$ é um isomorfismo linear.

Pelo Lema 2.1.16, existe algum $V_{3} \in \mathcal{H}^{n}$ tal que

$$
\left(\mathcal{L}_{R} V_{3}\right)(x, y)=-x P_{2}(x, y)-y Q_{2}(x, y)
$$

Com esta escolha de $V_{3}$, os termos de ordem 3 em Eq. (2.1.38) se anulam e portanto temos:

$$
\begin{aligned}
\mathcal{L}_{X} V(x, y)= & x P_{3}(x, y)+y Q_{3}(x, y)+V_{3 x}(x, y) P_{2}(x, y)+V_{3 y}(x, y) Q_{2}(x, y) \\
& +\left(\mathcal{L}_{R} V_{4}\right)(x, y)+O\left(\left\|x^{2}+y^{2}\right\|^{\frac{5}{2}}\right) .
\end{aligned}
$$

Lema 2.1.17. Se $n=2 k, k \in \mathbb{N}^{*}$, então o núcleo da transformação linear $\mathcal{L}_{R}: \mathcal{H}^{n} \rightarrow \mathcal{H}^{n}$ tem dimensão um e é gerado por $\left(x^{2}+y^{2}\right)^{k} \in \mathcal{H}^{n}$.

Por este lema, existe um polinômio homogêneo $V_{4} \in \mathcal{H}^{n}$, tal que a Eq. (2.1.39) assume a forma:

$$
\mathcal{L}_{X} V(x, y)=L_{4}\left(x^{2}+y^{2}\right)^{2}+O\left(\left\|x^{2}+y^{2}\right\|^{\frac{5}{2}}\right)
$$

onde $L_{4}$ é uma constante com respeito às variáveis $x$ e $y$.

Se $L_{4} \neq 0$, então a função

$$
V(x, y)=\frac{1}{2}\left(x^{2}+y^{2}\right)+V_{3}(x, y)+V_{4}(x, y)
$$

determina a estabilidade da origem. Mais precisamente, se $L_{4}<0$, então $V$ é uma função de Lyapunov em alguma vizinhança da origem e o ponto de equilíbrio é assintoticamente estável. Se $L_{4}>0$, então o ponto de equilíbrio é instável.

Observação 2.1.18. Este resultado sobre estabilidade não exige que o campo de vetores $X$ seja analítico. Além disso, os cálculos formais com $V$ são justificados, pois a função de Lyapunov dada pela expressão (2.1.40), que é um requisito para aplicação do Teorema 2.1.15, é, de fato, um polinômio.

Por outro lado, se $L_{4}=0$, então, pelo mesmo procedimento feito anteriormente, podemos obter uma nova expressão de $V$, tal que o termo líder de $\mathcal{L}_{X}$ é $L_{6}\left(x^{2}+y^{2}\right)^{3}$ e assim sucessivamente.

Os teoremas a seguir fornecem resultados bastante úteis com respeito a estabilidade. 
Teorema 2.1.19. Se $L_{2 n}=0, n=2, \ldots, N$, mas $L_{2 N+2} \neq 0$, então a estabilidade do ponto singular na origem está determinada. Mais precisamente, se $L_{2 N+2}<0$, então a origem é assintoticamente estável. Se $L_{2 N+2}>0$, a origem é instável.

Teorema 2.1.20 (Centro de Lyapunov). Se o campo vetorial $X$ é analítico e $L_{2 n}=$ $0, \forall n \geq 2$, então a origem é um centro. Além disso, a série que define $V$ é convergente numa vizinhança da origem e representa uma função cujos conjuntos de nivel contêm as órbitas da equação diferencial correspondente ao campo $X$.

Os Teoremas 2.1.19 e 2.1.20 e o algoritmo desenvolvido para calcular a expressão de $V$ nos fornecem um método para investigar a estabilidade em sistemas analíticos com singularidade não degenerada.

O resultado a seguir nos permite estabelecer uma relação entre os coeficientes $L_{2 n}$ da série de $V$ e a constante de Lyapunov definida em 2.1.12.

Lema 2.1.21. Sejam $V_{2 a+1}, a \in \mathbb{N}$ a $k$-ésima constante de Lyapunov para o sistema (2.1.35), e $L_{2 b}, b \geq 2$ os coeficientes de $V$ construídos como descrito anteriormente. Se $k \in \mathbb{N}^{*}$ e $L_{2 j}=0, j=1, \ldots, k-1$, então $V_{2 k-1}=2 \pi L_{2 k}$.

O seguinte teorema é consequência direta das construções feitas até o momento.

Teorema 2.1.22. Seja o sistema diferencial quadrático planar

$$
\left\{\begin{array}{l}
\dot{x}=\lambda_{1} x-y-\lambda_{3} x^{2}+\left(2 \lambda_{2}+\lambda_{5}\right) x y+\lambda_{6} y^{2} \\
\dot{y}=x+\lambda_{1} y+\lambda_{2} x^{2}+\left(2 \lambda_{3}+\lambda_{4}\right) x y-\lambda_{2} y^{2}
\end{array}\right.
$$

$\operatorname{com} \lambda_{1}=0$. Então, as três primeiras constantes de Lyapunov (a menos de uma constante $2 \pi)$, não nulas, são dadas por:

1. $V_{1}=-\lambda_{5}\left(\lambda_{3}-\lambda_{6}\right)$;

2. $V_{3}=\lambda_{2} \lambda_{4}\left(\lambda_{3}-\lambda_{6}\right)\left(\lambda_{4}+5 \lambda_{3}-5 \lambda_{6}\right)$;

3. $V_{5}=-\lambda_{2} \lambda_{4}\left(\lambda_{3}-\lambda_{6}\right)^{2}\left(\lambda_{3} \lambda_{6}-\lambda_{2}^{2}-2 \lambda_{6}^{2}\right)$.

A seguir, apresentamos os resultados obtidos por Bautin [20] na investigação dos sistemas diferenciais polinomiais de grau 2. Desses estudos, podemos compreender melhor a observação anterior a respeito das obstruções no estudo de casos mais gerais. 


\subsubsection{Campos vetoriais quadráticos no plano}

O problema do centro-foco para campos de vetores quadráticos planares foi completamente solucionado por Frommer [21] e Bautin [10], embora sua investigação tenha recebido contribuições relevantes de diversos outros matemáticos (o método de Kapteyn, por exemplo, que investigou o problema do centro-foco cerca de 20 anos antes de Frommer e Bautin oferecerem sua solução, pode ser encontrado em [20] pp. 183-193).

Nesta seção, apresentaremos um teorema devido a Bautin, que possibilita caracterizar os centros em campos vetoriais quadráticos no plano. Destacamos que o método desenvolvido por Bautin permite não apenas resolver o problema do centro-foco para esses sistemas, mas oferece ferramentas para investigar a bifurcação de ciclos limites. Nesta dissertação, entretanto, não trataremos desse estudo (o método de Bautin para problemas de bifurcação pode ser consultado em [47]).

Considere o sistema diferencial:

$$
\left\{\begin{array}{l}
\dot{x}=a_{20} x^{2}+a_{11} x y+a_{02} y^{2} \\
\dot{y}=b_{20} x^{2}+b_{11} x y+b_{02} y^{2}
\end{array}\right.
$$

onde

$$
\left(a_{10}-b_{01}\right)^{2}+4 a_{01} b_{10}<0
$$

Esta é a forma geral de um sistema diferencial polinomial planar de grau 2, com uma singularidade isolada na origem, tal que sua parte linear tem um centro em $(0,0)$. De fato, é fácil ver que a origem é ponto singular de (2.1.41). Mais, se houvessem termos constantes na Eq. (2.1.41), então $(0,0)$ não seria ponto singular do sistema. Por outro lado, da condição (2.1.42) segue que a parte linear de Eq. (2.1.41) tem autovalores complexos conjugados $\lambda_{1,2}=\alpha \pm i \beta, \beta \neq 0$.

Deste modo, a Eq. (2.1.41) satisfaz as hipóteses da Proposição 1.2.8, podendo ser escrita na forma:

$$
\left\{\begin{array}{l}
\frac{d \zeta}{d \tau}=\alpha \zeta-\beta \eta+A_{20} \zeta^{2}+A_{11} \zeta \eta+A_{02} \eta^{2} \\
\frac{d \eta}{d \tau}=\beta \zeta+\alpha \eta+B_{20} \zeta^{2}+B_{11} \zeta \eta+B_{02} \eta^{2}
\end{array}\right.
$$


Efetuando uma mudança de coordenadas do tipo homotetia-rotação

$$
\left\{\begin{array}{l}
x=h \zeta+k \eta \\
y=-k \zeta+h \eta
\end{array}\right.
$$

os termos de primeiro grau do sistema permanecem invariantes. O sistema, então, assume a seguinte forma:

$$
\left\{\begin{array}{l}
\frac{d x}{d \tau}=\alpha x-\beta y+A_{20}^{*} x^{2}+A_{11}^{*} x y+A_{02}^{*} y^{2}, \\
\frac{d y}{d \tau}=\beta x+\alpha y+B_{20}^{*} x^{2}+B_{11}^{*} x y+B_{02}^{*} y^{2} .
\end{array}\right.
$$

É possível escolher $h$ e $k$ tais que $B_{20}^{*}+B_{02}^{*}=0$, a menos que $B_{20}+B_{02}=A_{20}+A_{02}=0$ (para maiores detalhes consultar [20] pp. 184-193), de tal forma que o sistema finalmente pode ser escrito como

$$
\left\{\begin{array}{l}
\frac{d x}{d t}=\lambda_{1} x-y-\lambda_{3} x^{2}+\left(2 \lambda_{2}+\lambda_{5}\right) x y+\lambda_{6} y^{2} \equiv P(x, y) \\
\frac{d y}{d t}=x+\lambda_{1} y+\lambda_{2} x^{2}+\left(2 \lambda_{3}+\lambda_{4}\right) x y-\lambda_{2} y^{2} \equiv Q(x, y) .
\end{array}\right.
$$

onde $\beta \tau=t, \lambda_{1}=\frac{\alpha}{\beta}, \beta \neq 0$ por (2.1.42).

Os coeficientes $\lambda_{4}, \lambda_{5}$ são introduzidos na equação da forma dada para simplificar as condições para um centro na origem.

Portanto, o sistema diferencial planar de grau 2 (2.1.41), com uma singularidade isolada na origem e que satisfaz a condição (2.1.42), pode ser transformado em um sistema da forma (2.1.43). De agora em diante, vamos nos referir a esta forma.

Teorema 2.1.23 (Teorema de Bautin). [10] A origem do sistema (2.1.43) é um centro se, e somente se, uma das seguintes condições for satisfeita:

$$
\begin{aligned}
& \text { 1. } \lambda_{1}=0, \lambda_{3}-\lambda_{6}=0 ; \\
& \text { 2. } \lambda_{1}=\lambda_{2}=\lambda_{5}=0 \\
& \text { 3. } \lambda_{1}=\lambda_{4}=\lambda_{5}=0 ;
\end{aligned}
$$


4. $\lambda_{1}=\lambda_{5}=\lambda_{4}+5\left(\lambda_{3}-\lambda_{6}\right)=\lambda_{3} \lambda_{6}-\lambda_{2}^{2}-2 \lambda_{6}^{2}=0$.

A demonstração completa deste teorema pode ser encontrada em [10, 20, 12].

A seguir, discutimos algumas etapas da demonstração.

Suponhamos inicialmente, que a origem do sistema (2.1.43) é um centro. Na Seção 2.1.2, mostramos a relação entre as constantes de Lyapunov e os coeficientes da expansão da série de Taylor da Aplicação de Poincaré. Se a origem do sistema é um centro, então pela expressão (2.1.33), segue que a aplicação de primeiro retorno é a identidade. Consequentemente, as constantes de Lyapunov são todas nulas. Em particular, as três primeiras constantes de Lyapunov são nulas, isto é, $V_{1}=V_{3}=V_{5}=0$. Pelo Teorema 2.1.22, as constantes de Lyapunov do sistema (2.1.43) são dadas por:

1. $V_{1}=-\lambda_{5}\left(\lambda_{3}-\lambda_{6}\right)$

2. $V_{3}=\lambda_{2} \lambda_{4}\left(\lambda_{3}-\lambda_{6}\right)\left(\lambda_{4}+5 \lambda_{3}-5 \lambda_{6}\right)$

3. $V_{5}=-\lambda_{2} \lambda_{4}\left(\lambda_{3}-\lambda_{6}\right)^{2}\left(\lambda_{3} \lambda_{6}-\lambda_{2}^{2}-2 \lambda_{6}^{2}\right)$.

Usando que $V_{1}=V_{3}=V_{5}=0$, obtemos as quatro condições dadas no Teorema 2.1.23.

Suponhamos agora, que as condições $1-4$ do Teorema 2.1.23 são satisfeitas.

Escrevendo o sistema (2.1.43) em coordenadas polares $x=r \cos (\theta), y=r \operatorname{sen}(\theta)$ :

$$
\left\{\begin{aligned}
\dot{r}= & \lambda_{1} r+\left(3 \lambda_{2}+\lambda_{5}\right) r^{2} \operatorname{sen}(\theta) \cos ^{2}(\theta)+\left(2 \lambda_{3}+\lambda_{4}+\lambda_{6}\right) r^{2} \operatorname{sen}^{2}(\theta) \cos (\theta)-\lambda_{2} r^{2} \operatorname{sen}^{3}(\theta) \\
& -\lambda_{3} r^{2} \cos ^{3}(\theta), \\
\dot{\theta}= & 1+\left(3 \lambda_{3}+\lambda_{4}\right) r \operatorname{sen}(\theta) \cos ^{2}(\theta)-\left(3 \lambda_{2}+\lambda_{5}\right) r \operatorname{sen}^{2}(\theta) \cos (\theta)+\lambda_{2} r \cos ^{3}(\theta) \\
& -\lambda_{6} r \operatorname{sen}^{3}(\theta) .
\end{aligned}\right.
$$

Segue que, numa vizinhança suficientemente próxima da origem, a expressão abaixo é analítica:

$$
R(r, \theta)=\frac{d r}{d \theta}=\frac{r \lambda_{1}+r^{2} \chi_{1}(\theta)}{1+r \chi_{2}(\theta)}
$$

onde

$$
\begin{aligned}
\chi_{1}= & -\lambda_{3} \cos ^{3}(\theta)+\left(3 \lambda_{2}+\lambda_{5}\right) \operatorname{sen}(\theta) \cos ^{2}(\theta)+\left(2 \lambda_{3}+\lambda_{4}+\lambda_{6}\right) \operatorname{sen}^{2}(\theta) \cos (\theta)-\lambda_{2} \operatorname{sen}^{3}(\theta) \\
& -\lambda_{3} \cos ^{3}(\theta), \\
\chi_{2}= & \left(3 \lambda_{3}+\lambda_{4}\right) \operatorname{sen}(\theta) \cos ^{2}(\theta)-\left(3 \lambda_{2}+\lambda_{5}\right) \operatorname{sen}^{2}(\theta) \cos (\theta)+\lambda_{2} \cos ^{3}(\theta)-\lambda_{6} \operatorname{sen}^{3}(\theta) .
\end{aligned}
$$


Portanto, podemos expandir $R(r, \theta)$ em potências de $r$ :

$$
R(r, \theta)=R_{1} r+R_{2}(\theta) r^{2}+R_{3}(\theta) r^{3}+\ldots
$$

onde

$R_{1}=\lambda_{1}$

$R_{2}=\chi_{1}(\theta)-\lambda_{1} \chi_{2}(\theta)$

$R_{k}=(-1)^{k} \chi_{1}(\theta) \chi_{2}(\theta)^{k-2}+(-1)^{k-1} \lambda_{1} \chi_{2}(\theta)^{k-1}, \quad k \geq 2$,

isto é, $R_{k}(k \geq 2)$ são polinômios em $\operatorname{sen}(\theta)$ e $\cos (\theta)$ e nos parâmetros $\lambda_{i}, \quad 1 \leq i \leq 6$.

Se $r(\theta)$ é uma solução de (2.1.44), satisfazendo a condição inicial $r(0)=r_{0}$, então numa vizinhança da origem, podemos expandir essa solução em série de potências:

$$
r=v_{1}\left(\theta, \lambda_{i}\right) r_{0}+v_{2}\left(\theta, \lambda_{i}\right) r_{0}^{2}+v_{3}\left(\theta, \lambda_{i}\right) r_{0}^{3}+\ldots
$$

Para $\theta=0$, temos $r(0)=r_{0}$ e desta forma,

$$
r_{0}=r(0)=v_{1}\left(0, \lambda_{i}\right) r_{0}+v_{2}\left(0, \lambda_{i}\right) r_{0}^{2}+v_{3}\left(0, \lambda_{i}\right) r_{0}^{3}+\ldots
$$

e consequentemente, $v_{1}\left(0, \lambda_{i}\right)=1$ e $v_{k}\left(0, \lambda_{i}\right)=0 \forall k \geq 2$.

Agora, fazendo a substituição de (2.1.46) em (2.1.45) e comparando os coeficientes das potências de $r$ obtemos:

$$
\left\{\begin{array}{l}
\frac{d V_{1}}{d \theta}=v_{1} R_{1} \\
\frac{d V_{2}}{d \theta}=v_{2} R_{1}+v_{1}^{2} R_{2} \\
\frac{d V_{3}}{d \theta}=v_{3} R_{1}+2 v_{1} v_{2} R_{2}+v_{1}^{3} R_{3}
\end{array}\right.
$$

e assim por diante.

Se $\theta=2 \pi$, de Eq. (2.1.46) temos:

$$
r(2 \pi)=v_{1}\left(2 \pi, \lambda_{i}\right) r_{0}+v_{2}\left(2 \pi, \lambda_{i}\right) r_{0}^{2}+v_{3}\left(2 \pi, \lambda_{i}\right) r_{0}^{3}+\ldots
$$

A proposição a seguir nos fornece um resultado importante para completarmos a de- 
monstração.

Proposição 2.1.24. As funções $v_{k}\left(2 \pi, \lambda_{i}\right)$, em (2.1.48), são funções integrais dos coeficientes $\lambda_{i}$. Se $\lambda_{1}=0$, então $v_{k}\left(2 \pi, \lambda_{i}\right)$ se tornam polinômios homogêneos de grau $(k-1)$ nos parâmetros $\lambda_{i}, i=2, \ldots, 6$. Além disso:

$v_{1}\left(2 \pi, \lambda_{i}\right)=e^{2 \pi \lambda_{1}}$

$v_{2}\left(2 \pi, \lambda_{i}\right)=\lambda_{1} \psi_{2}^{(1)}$;

$v_{3}\left(2 \pi, \lambda_{i}\right)=\bar{v}_{3}+\lambda_{1} \psi_{3}^{(1)}$;

$v_{4}\left(2 \pi, \lambda_{i}\right)=\bar{v}_{3} \psi_{4}^{(3)}+\lambda_{1} \psi_{4}^{(1)}$;

$v_{5}\left(2 \pi, \lambda_{i}\right)=\bar{v}_{5}+\bar{v}_{3} \psi_{5}^{(3)}+\lambda_{1} \psi_{5}^{(1)}$;

$v_{6}\left(2 \pi, \lambda_{i}\right)=\bar{v}_{5} \psi_{6}^{(5)}+\bar{v}_{3} \psi_{6}^{(3)}+\lambda_{1} \psi_{6}^{(1)}$

$v_{7}\left(2 \pi, \lambda_{i}\right)=\bar{v}_{7}+\bar{v}_{5} \psi_{7}^{(5)}+\bar{v}_{3} \psi_{7}^{(3)}+\lambda_{1} \psi_{7}^{(1)}$;

$v_{k}\left(2 \pi, \lambda_{i}\right)=\bar{v}_{7} \psi_{k}^{(7)}+\bar{v}_{5} \psi_{k}^{(5)}+\bar{v}_{3} \psi_{k}^{(3)}+\lambda_{1} \psi_{k}^{(1)}, \quad k>7$,

onde

$\bar{v}_{3}=-\frac{1}{4} \pi \lambda_{5}\left(\lambda_{3}-\lambda_{6}\right)$,

$\bar{v}_{5}=\frac{1}{21} \pi \lambda_{2} \lambda_{4}\left(\lambda_{3}-\lambda_{6}\right)\left(\lambda_{4}+5 \lambda_{3}-5 \lambda_{6}\right)$,

$\bar{v}_{7}=\frac{25}{32} \pi \lambda_{2} \lambda_{4}\left(\lambda_{3}-\lambda_{6}\right)^{2}\left(\lambda_{3} \lambda_{6}-2 \lambda_{6}^{2}-\lambda_{2}^{2}\right)$

$e \psi_{k}^{(j)}$ são funçôes integrais dos $\lambda_{i}$.

Vale observar que, por recorrência e pela aplicação das condições $1-4$ do Teorema 2.1.23, obtemos as expressões de $v_{1}$ a $v_{7}$. Para a prova de $v_{k}, k \geq 8$, sugerimos consultar $[53,12]$.

Na Seção 2.1.2, mostramos a relação entre as constantes de Lyapunov e os coeficientes da expansão da série de Taylor da aplicação de Poincaré (vide (2.1.33)). Observe que as funções $v_{i}\left(2 \pi, \lambda_{i}\right), \quad i \geq 2$ definidas acima, são justamente os coeficientes da expansão da série de Taylor da aplicação de Poincaré dada na Eq. (2.1.46), para $\theta=2 \pi$. Portanto, satisfeitas as condições 1-4 do Teorema 2.1.23, segue pela Proposição 2.1.24 que, todas as constantes de Lyapunov são nulas e consequentemente, a origem de (2.1.43) é um centro.

\subsection{Singularidades degeneradas linearmente nulas}

Singularidades degeneradas podem ser do tipo nilpotente, elementares ou linearmente nulas - cf. Definição 1.1.10. Em geral, para pontos singulares degenerados o problema do 
centro-foco pode ser decomposto em duas partes - cf. [1, 38]:

1. problema da monodromia, que consiste em determinar sob quais condições o ponto singular é monodrômico;

2. problema da estabilidade, que visa decidir, uma vez conhecido que o ponto singular é monodrômico, se é um centro ou um foco.

De acordo com Andronov at al. [5], pontos singulares elementares podem ser apenas do tipo sela, nó, ou sela-nó e portanto, não são monodrômicos. O caso de singularidades nilpotentes será estudado, em detalhes, no Capítulo 3.

A caracterização de centros para pontos singulares degenerados com parte linear identicamente nula ainda é um caso em aberto. Os resultados obtidos até o momento se referem a famílias específicas de sistemas diferenciais - cf. [38, 24, 9].

Em geral, a investigação do problema do centro-foco para este tipo de singularidade se divide em duas modalidades, dependendo da existência ou não de direções características. No caso em que o ponto singular não tem direções características, sua estabilidade pode ser investigada por meio de ferramentas como as constantes de Lyapunov generalizadas, dentre outras. As principais dificuldades que surgem são do tipo computacional - cf. [27]. Na presença de direções características, diversas técnicas vem sendo desenvolvidas, mas nenhuma tão eficiente quanto as técnicas usadas nos casos de singularidades não degeneradas e nilpotentes - cf. [38].

Apresentamos a seguir, alguns resultados extraídos de [29].

Seja $p$ uma singularidade degenerada com parte linear identicamente nula que, sem perda de generalidade, consideraremos como sendo a origem, de um sistema diferencial analítico. Então, de acordo com a Proposição 1.2.11, este sistema pode ser escrito na forma

$$
\left\{\begin{array}{l}
\dot{x}=F(x, y), \\
\dot{y}=G(x, y),
\end{array}\right.
$$

onde $F(x, y)$ e $G(x, y)$ são funções analíticas numa vizinhança da origem, com $F(0,0)=$ $G(0,0)=D F(0,0)=D G(0,0)=0$.

No que se segue, um centro de um sistema diferencial analítico em $\mathbb{R}^{2}$ é chamado de degenerado se puder ser escrito na forma (2.2.1).

Com base nos resultados que serão posteriormente apresentados na Seção 3.2, para os chamados centros nilpotentes, apresentamos a seguinte definição: 
Definição 2.2.1. Suponhamos que a origem do sistema diferencial analítico (2.2.1) seja um centro. Dizemos que este sistema é limite de centros lineares se existem funções analiticas $G_{1}$ e $G_{2}$ em $x, y, e \varepsilon$, sem termos constantes e lineares em $x$ e $y$, tais que o sistema

$$
\dot{x}=\varepsilon y+F_{1}(x, y)+\varepsilon G_{1}(x, y, \varepsilon), \quad \dot{y}=-\varepsilon x+F_{2}(x, y)+\varepsilon G_{2}(x, y, \varepsilon)
$$

tem um centro linear na origem, para todo $\varepsilon \neq 0$ suficientemente pequeno.

A partir desta definição, enunciamos o seguinte teorema:

Teorema 2.2.2. Suponha que a origem do sistema diferencial analítico real (2.2.1) é monodrômica, e que o sistema é limite de centros lineares da forma (2.2.2). Suponha ainda que não existe ponto singular de (2.2.2) tendendo para a origem, quando $\varepsilon$ tende a zero. Então, o sistema (2.2.1) tem um centro na origem.

A demonstração deste teorema é análoga àquela do Teorema 3.2.5, da Seção 3.2. A condição de ausência de pontos singulares tendendo à origem, quando $\varepsilon$ tende a zero, pode ser facilmente verificada usando os termos de menor ordem do sistema perturbado (2.2.2).

Uma das dificuldades na investigação do problema do centro-foco para este tipo de singularidades vem do fato de que este problema pode não ser algebricamente solúvel, isto é, pode não existir uma sequência infinita de expressões polinomiais independentes envolvendo os coeficientes do sistema, de tal forma que seu anulamento simultâneo garanta a existência de um centro - cf. [32].

O próximo resultado trata de centros degenerados que são limite de centros lineares.

Teorema 2.2.3. Para um centro analítico degenerado, as seguintes afirmações são válidas:

(a) Um centro degenerado hamiltoniano é sempre limite de centros hamiltonianos lineares;

(b) Um centro degenerado reversível no tempo é sempre limite de centros lineares reversiveis no tempo; 
(c) Existem centros degenerados que não são hamiltonianos ou reversíveis no tempo, que são limite de centros lineares;

(d) Centros degenerados insolúveis algebricamente não são limite de centros lineares;

(e) Existem centros degenerados solúveis algebricamente que não são limite de centros lineares;

(f) Existem centros degenerados com direções características que são limite de centros degenerados sem direções características.

A demonstração deste teorema encontra-se em [29].

Como mencionamos anteriormente, há vários outros resultados que tratam do estudo de centros linearmente nulos e não são discutidos nesta dissertação. 


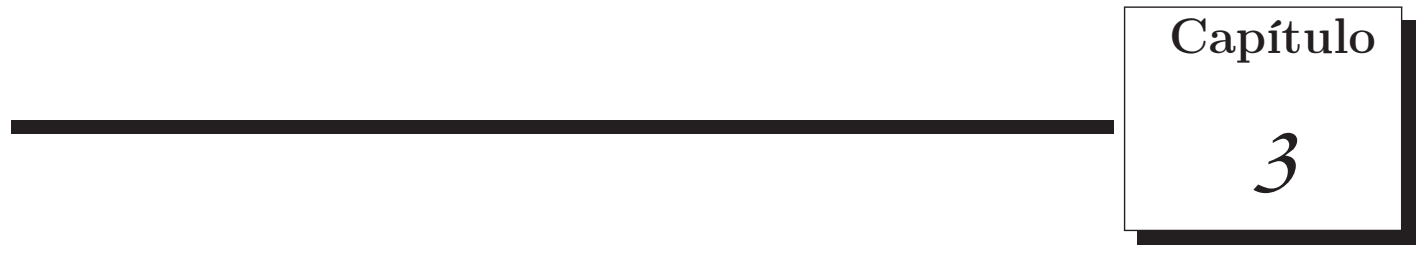

\section{Problema do centro-foco: singularidades nilpotentes}

Neste capítulo, vamos investigar detalhadamente o problema do centro-foco em sistemas diferenciais analíticos com singularidades nilpotentes. Este problema ainda é bastante estudado, uma vez que, não existe até o momento, um algoritmo comparável ao método de Lyapunov usado no estudo de campos com singularidades não-degeneradas cf. [29].

Duas técnicas bastante distintas serão apresentadas. A primeira, desenvolvida por Álvarez \& Gasull - cf. [1, 2], utiliza a teoria das formas normais, e aborda o problema da maneira clássica, dividindo-o na investigação da monodromia e no estudo da estabilidade. O segundo método, devido à Giacomini, Giné \& Llibre - cf. [29], investiga os sistemas diferenciais analíticos com centros nilpotentes como limite de sistemas com centros do tipo linear.

\subsection{Método de Álvarez-Gasull}

Conforme mencionado no Capítulo 2, para pontos singulares degenerados, o problema do centro-foco usualmente é decomposto em duas partes: 
1. problema da monodromia, que consiste em determinar sob quais condições o ponto singular é monodrômico;

2. problema da estabilidade, que visa decidir, uma vez conhecido que o ponto singular é monodrômico, se é um centro ou um foco.

O problema da monodromia para pontos singulares deste tipo foi solucionado por Andreev [3], em 1958 e o problema da estabilidade por Moussu [41], em 1982. Nas próximas seções, discutimos cada um destes problemas, apresentando demonstrações mais elegantes dos teoremas de Andreev e Moussu, que utilizam a teoria das formas normais e a técnica de blow-up $(1, n)$-quasehomogêneo, dentre outros.

\subsubsection{Problema da monodromia}

A solução para o problema da monodromia, determinada por Andreev [3], consiste em analisar todas as possíveis configurações topológicas que um sistema diferencial não-linear planar, com singularidade degenerada nilpotente, pode assumir numa vizinhança desta singularidade. Esta análise extensiva permitiu a Andreev obter condições necessárias e suficientes para caracterização da monodromia.

A demonstração deste resultado, apesar de clássica, é um tanto extensa e trabalhosa. Optamos, então, por uma demonstração mais elegante e curta, obtida por Álvarez e Gasull [1] em 2005.

Seja o sistema diferencial analítico no plano

$$
\left\{\begin{array}{l}
\dot{x}=P(x, y), \\
\dot{y}=Q(x, y),
\end{array}\right.
$$

e seja $p$ um ponto singular nilpotente deste sistema. Sem perda de generalidade, podemos assumir que $p$ é a origem (vide Lema 1.2.7). Pela Proposição 1.2.10, o sistema (3.1.1) pode ser escrito na forma

$$
\left\{\begin{array}{l}
\dot{x}=y+X_{2}(x, y) \\
\dot{y}=Y_{2}(x, y)
\end{array}\right.
$$

onde $X_{2}, Y_{2}$ são funções analíticas cujas séries de potências começam, pelo menos, com termos de grau 2. Então, vale o seguinte resultado: 
Teorema 3.1.1 (Teorema da Monodromia para Singularidades Nilpotentes). [3]

Considere o sistema (3.1.2), e assuma que a origem seja uma singularidade isolada. Defina as funções:

$$
\begin{aligned}
f(x) & :=Y_{2}(x, F(x)) \\
& =a x^{\alpha}+O\left(\|x\|^{\alpha+1}\right), a \neq 0, \alpha \geq 2 \\
\phi(x) & :=\left.\operatorname{div}\left(y+X_{2}(x, y), Y_{2}(x, y)\right)\right|_{y=F(x)} \\
& =\left.\left[\frac{\partial\left(y+X_{2}(x, y)\right)}{\partial x}+\frac{\left.\partial Y_{2}(x, y)\right)}{\partial y}\right]\right|_{y=F(x)}
\end{aligned}
$$

onde $\phi(x)=b x^{\beta}+O\left(\|x\|^{\beta+1}\right), b \neq 0, \beta \geq 1$ ou $\phi \equiv 0$. A função $y=F(x)$ é a solução de $y+X_{2}(x, y)=0$ passando por $(0,0)$.

Então, a origem de (3.1.2) é monodrômica se, e somente se, a é negativo, a é um número natural ímpar ( $\alpha=2 n-1$, para algum $n)$, e vale uma das três condições abaixo:

(i) $\beta>n-1$;

(ii) $\beta=n-1$ e $b^{2}+4 a n<0$;

(iii) $\phi \equiv 0$.

Este teorema, que é o principal resultado desta seção, caracteriza a monodromia de singularidades isoladas em sistemas nilpotentes. Para sua demonstração, são necessárias algumas ferramentas adicionais que apresentamos a seguir.

Definição 3.1.2. [6] Seja $f: \mathbb{R}^{n} \rightarrow \mathbb{R}^{n}$ uma função diferenciável, tal que $f(0)=0$, e seja $p$ um valor regular numa vizinhança da origem. Definimos o indice de $f$ na origem como sendo

$$
\operatorname{ind}_{0}(f)=\sum_{x \in \mathbb{R}^{n}: x \in f^{-1}(p)} \operatorname{sgn}\left(J_{f}(x)\right)
$$

onde $J_{f}(x)$ denota o determinante da matriz jacobiana de $f$ no ponto $x$ e sgn é a função sinal.

O próximo resultado, devido a Poincaré - cf. [6], simplifica o cálculo do índice no caso planar. 
Proposição 3.1.3. Seja $C$ uma curva fechada simples em $\mathbb{R}^{2}$ e $\mathrm{X}$ um campo vetorial definido em uma região aberta simplesmente conexa de $\mathbb{R}^{2}$ contendo C. Seja L uma reta do plano. Suponha que existe um número finito de pontos $M_{k}, k=1, \ldots, n$ de $C$ tais que o vetor $\mathrm{X}\left(M_{k}\right)$ é paralelo a $L$. Seja $M$ um ponto que percorre a curva $C$ no sentido horário e p (respectivamente q) o número de pontos $M_{k}$, tais que $\mathrm{X}(M)$ intercepta a direção $L$ no sentido horário (respectivamente no sentido anti-horário). Então, o índice de $\mathrm{X}$ em $C$ é $\frac{p-q}{2}$. Adicionalmente, o indice de $\mathrm{X}$ em qualquer ponto crítico isolado $p$ é o índice de $\mathrm{X}$ em qualquer curva fechada simples que não possui pontos críticos sobre seu traço e que contém apenas o ponto crítico $p$ em seu interior.

Exemplo 3.1.4. O indice de um campo vetorial analítico em um ponto crítico isolado monodrômico é +1 .

De fato, numa vizinhança suficientemente pequena de um ponto crítico isolado monodrômico de um campo vetorial analítico, as órbitas não possuem direção característica (observe que, de acordo com [33], uma singularidade monodrômica de um campo vetorial analítico é um centro ou um foco).

Logo, tomando-se qualquer curva fechada simples $\gamma$ na vizinhança do ponto crítico $e$ fixada uma direção L, ao percorrermos esta curva com um ponto $M$ no sentido horário, existirão sempre apenas dois pontos $M_{1}$ e $M_{2}$ na curva tais que $\mathrm{X}(M)$ intercepta a direção $L$ no sentido horário, e nenhum ponto em que $\mathrm{X}(M)$ intercepta a direção $L$ no sentido anti-horário, de modo que $p=2$ e $q=0$. Ou seja, o índice é +1 . A figura a seguir ilustra o procedimento:

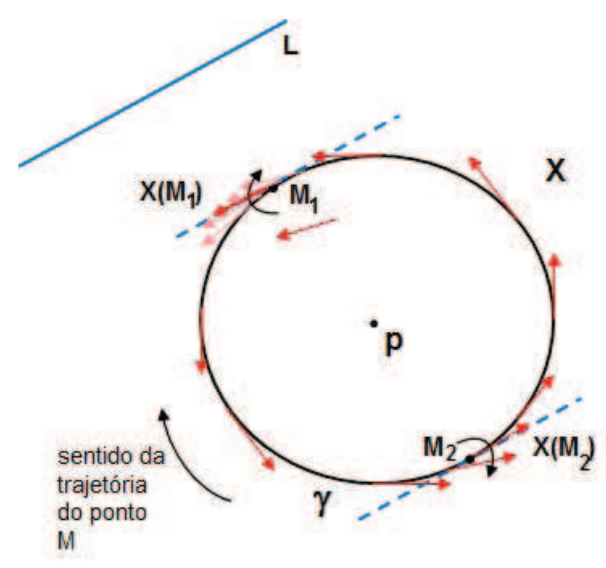

Figura 3.1: Índice de um campo vetorial analítico com monodromia 
O próximo lema nos mostra como a técnica de blow-up (1,n)-quasehomogêneo - cf. 1.4, é eficiente no estudo do problema do centro-foco.

Lema 3.1.5. Considere o seguinte sistema diferencial planar

$$
\left\{\begin{array}{l}
\dot{x}=y\left(-1+X_{1}(x, y)\right) \\
\dot{y}=f(x)+\phi(x) y+Y_{0}(x, y) y^{2}
\end{array}\right.
$$

onde

$$
\begin{aligned}
& X_{1}(x, y)=\sum_{i+j \geq 1} d_{i j} x^{i} y^{j}, \\
& f(x)=x^{2 n-1}+\sum_{i \geq 0} a_{i} x^{2 n+i}, \\
& \phi(x)=b x^{\beta}+\sum_{i \geq 1} b_{i} x^{\beta+i}, \\
& Y_{0}(x, y)=\sum_{i+j \geq 0} e_{i j} x^{i} y^{j},
\end{aligned}
$$

com $\beta \geq 1$ um número natural arbitrário. Tome o valor de $n$ dado pela expressão $f(x)=$ $x^{2 n-1}+O\left(\|x\|^{2 n}\right)$.

Então, a expressão de (3.1.3) em coordenadas $(1, n)$-quasehomogêneas - ver (1.4.7) é dada por:

$$
\left\{\begin{array}{l}
\dot{r}=P_{1}(\theta) r^{\beta+1}+P_{2}(\theta) r^{n+1}+P_{3}(\theta) r^{n+2}+P_{4}(\theta) r^{n+3}+O\left(\|r\|^{n+4}\right), \\
\dot{\theta}=Q_{1}(\theta) r^{\beta}+Q_{2}(\theta) r^{n}+Q_{3}(\theta) r^{n+1}+O\left(\|r\|^{n+2}\right),
\end{array}\right.
$$

onde $P_{i}(\theta)=P_{i}(\operatorname{Cs}(\theta), \operatorname{Sn}(\theta))$, para $i=1, \ldots, 4$, e $Q_{i}(\theta)=Q_{i}(\operatorname{Cs}(\theta), \operatorname{Sn}(\theta))$, para $i=$ $1, \ldots, 3$ são definidos como se segue:

$$
\begin{aligned}
& P_{1}(x, y)=\mathcal{X}_{\{\beta \leq-1\}} b x^{\beta} y^{2}, \\
& P_{2}(x, y)=\left(a_{0}+d_{10}\right) x^{2 n} y+\mathcal{X}_{\{\beta=n\}} b x^{n} y^{2}+e_{00} y^{3}, \\
& P_{3}(x, y)=\left(a_{1}+d_{20}\right) x^{2 n+1} y+\left[\mathcal{X}_{\{\beta=n\}} b_{1}+\mathcal{X}_{\{\beta=n+1\}} b\right] x^{n+1} y^{2}+e_{10} x y^{3}+\mathcal{X}_{\{n=2\}} d_{01} x^{2 n-1} y^{2},
\end{aligned}
$$




$$
\begin{aligned}
P_{4}(x, y)= & \left(a_{2}+d_{30}\right) x^{2 n+2} y+\left[\mathcal{X}_{\{\beta=n\}} b_{2}+\mathcal{X}_{\{\beta=n+1\}} b_{1}+\mathcal{X}_{\{\beta=n+2\}} b\right] x^{n+2} y^{2}+e_{20} x^{2} y^{3} \\
& +\mathcal{X}_{\{n=2\}}\left[d 11 x^{2 n} y^{2}+e_{01} y^{4}\right]+\mathcal{X}_{\{n=3\}}\left[d_{01} x^{2 n-1} y\right] \\
Q_{1}(x, y)= & 1+\mathcal{X}_{\{\beta \leq n-1\}} b x^{\beta+1} y, \\
Q_{2}(x, y)= & a_{0} x^{2 n+1}+\left(e_{00}-n d_{10}\right) x y^{2}+\mathcal{X}_{\{\beta=n\}} b x^{n+1} y, \\
Q_{3}(x, y)= & a_{1} x^{2 n+2}+\left(e_{10}-n d_{20}\right) x^{2} y^{2}+\left[\mathcal{X}_{\{\beta=n\}} b_{1}+\mathcal{X}_{\{\beta=n+1\}} b\right] x^{n+2} y-\mathcal{X}_{\{n=2\}} 2 d_{01} y^{3}
\end{aligned}
$$

Demonstração. Substituindo as expressões de (1.4.7) e de (3.1.3) em (1.4.8) obtemos

$$
\begin{aligned}
r^{2 n-1} \dot{r}= & (r \operatorname{Cs}(\theta))^{2 n-1}\left\{r^{n} \operatorname{Sn}(\theta)\left[-1+\sum_{i+j \geq 1} d_{i j}(r \operatorname{Cs}(\theta))^{i}\left(r^{n} \operatorname{Sn}(\theta)\right)^{j}\right]\right\} \\
& +r^{n} \operatorname{Sn}(\theta)\left\{(r \operatorname{Cs}(\theta))^{2 n-1}+\sum_{i \geq 0} a_{i}(r \operatorname{Cs}(\theta))^{2 n+i}\right. \\
& +r^{n} \operatorname{Sn}(\theta)\left[b(r \operatorname{Cs}(\theta))^{\beta}+\sum_{i \geq 1} b_{i}(r \operatorname{Cs}(\theta))^{\beta+i}\right] \\
& \left.+\left(r^{n} \operatorname{Sn}(\theta)\right)^{2}\left[\sum_{i+j \geq 0} e_{i j}(r \operatorname{Cs}(\theta))^{i}\left(r^{n} \operatorname{Sn}(\theta)\right)^{j}\right]\right\}
\end{aligned}
$$

Efetuando-se os cálculos necessários, chega-se na seguinte expressão:

$$
\begin{aligned}
\dot{r}= & \sum_{i+j \geq 1} d_{i j} r^{n+i+n j} \operatorname{Cs}^{2 n+i-1}(\theta) \operatorname{Sn}^{j+1}(\theta)+\sum_{i \geq 0} a_{i} r^{n+i+1} \operatorname{Cs}^{2 n+i}(\theta) \operatorname{Sn}(\theta) \\
& +b r^{\beta+1} \operatorname{Cs}^{\beta}(\theta) \operatorname{Sn}^{2}(\theta)+\sum_{i \geq 1} b_{i} r^{\beta+i+1} \operatorname{Cs}^{\beta+i}(\theta) \operatorname{Sn}^{2}(\theta) \\
& +\sum_{i+j \geq 0} e_{i j} r^{n+i+n j+1} \operatorname{Cs}^{i}(\theta) \operatorname{Sn}^{j+3}(\theta)
\end{aligned}
$$

Destacamos que, considerando-se os índices em cada somatório, não existem termos em $r^{n}$ na expressão acima. Tomando-se $x=\operatorname{Cs}(\theta)$ e $y=\operatorname{Sn}(\theta)$, obtemos a expressão para $\dot{r}$ apresentada em (3.1.4).

A expressão para $\dot{\theta}$ é calculada de maneira similar e por isso, vamos omiti-la.

Lema 3.1.6. O sistema diferencial (3.1.2) pode ser escrito na forma

$$
\left\{\begin{array}{l}
\dot{x}=y\left(1+X_{1}(x, y)\right) \\
\dot{y}=f(x)+\phi(x) y+Y_{0}(x, y) y^{2}
\end{array}\right.
$$


com as funções $f$ e $\phi$ definidas no enunciado do Teorema 3.1.1. As funções $x_{1}$ e $Y_{0}$ são analíticas na origem. Além disso, $X_{1}(0,0)=0$.

Observe que, como a origem é uma singularidade isolada, a função $f(x)$ não pode ser identicamente nula. De fato, se $f(x) \equiv 0$, então todos os pontos da forma $(x, 0)$ seriam singularidades do sistema (3.1.5), e portanto a origem não seria singularidade isolada. Logo, $a \neq 0$.

Demonstração. Mostraremos que a mudança de coordenadas analítica a seguir, efetua a transformação desejada.

$$
\left\{\begin{array}{l}
x=x, \\
y=y_{1}+F(x),
\end{array}\right.
$$

onde $y=F(x)$ é a solução de $y+X_{2}(x, y)=0$ passando por $(0,0)$

De fato, aplicando (3.1.6) em (3.1.2), e usando que $F(x)=-X_{2}(x, F(x))$, pois $y=$ $F(x)$ é solução de $y+X_{2}(x, F(x))=0$, temos para $\dot{x}$ :

$$
\begin{aligned}
\dot{x} & =y_{1}+F(x)+X_{2}\left(x, y_{1}+F(x)\right) \\
& =y_{1}-X_{2}(x, F(x))+X_{2}\left(x, y_{1}+F(x)\right) \\
& =y_{1}+y_{1}\left(\frac{\left.X_{2}\left(x, y_{1}+F(x)\right)-X_{2}(x, F(x))\right)}{y_{1}}\right)
\end{aligned}
$$

Defina $X_{1}\left(x, y_{1}\right):=\frac{X_{2}\left(x, y_{1}+F(x)\right)-X_{2}(x, F(x))}{y_{1}}$.

A função $X_{1}$ é analítica. De fato, como $X_{2}$ é analítica por hipótese, podemos obter as expansões em séries de Taylor de $X_{2}\left(x, y_{1}+F(x)\right)$ e $X_{2}(x, F(x))$. Nestas séries, os termos da forma $a_{k} x^{k}, k \geq 2$ são idênticos e assim, se cancelam na expressão de $X_{1}$. Assim,

$$
X_{2}\left(x, y_{1}+F(x)\right)-X_{2}(x, F(x))=y_{1} \sum_{i+j \geq 1} x^{i} y_{1}^{j}
$$

e portanto,

$$
X_{1}\left(x, y_{1}\right)=\sum_{i+j \geq 1} x^{i} y_{1}^{j}
$$


Para $\dot{y}$, o argumento é semelhante. A seguir, fornecemos um esboço da demonstração:

$$
\begin{aligned}
& \dot{y}_{1}+\dot{F}(x)=Y_{2}\left(x, y_{1}+F(x)\right) \\
& \dot{y}_{1}+\frac{\partial F(x)}{\partial x} \dot{x}=Y_{2}\left(x, y_{1}+F(x)\right) \\
& \dot{y}_{1}=Y_{2}\left(x, y_{1}+F(x)\right)-\frac{\partial F(x)}{\partial x} y_{1}\left[1+X_{1}(x, y)\right]
\end{aligned}
$$

Observe que, como todas as funções na expressão acima são analíticas, usando suas respectivas expansões em série de Taylor é possível escrever $\dot{y}_{1}=p(x)+y_{1} q(x)+y_{1}^{2} r(x, y)$. Esta forma, entretanto, não necessariamente fornece as funções $\phi(x)$ e $Y_{0}$ do Lema 3.1.6.

Como $y=F(x)$ é solução de $y+X_{2}(x, F(x))=0$, temos, pelo Teorema da Função Implítica, $\frac{\partial F(x)}{\partial x}=-X_{2}(x, F(x))\left[1+\frac{\partial Y_{2}(x, F(x))}{\partial y}\right]^{-1}$.

Usando que $\left[1+\frac{\partial Y_{2}(x, F(x))}{\partial y}\right]^{-1}=\left[1-\frac{\partial Y_{2}(x, F(x))}{\partial y}+O\left(\|(x, y)\|^{2}\right)\right]$, e considerando a expansão de $Y_{2}\left(x, y_{1}+F(x)\right)$ em parcelas respectivamente independente de $y_{1}$, linear em $y_{1}$ e quadrada em $y_{1}$ e rearranjando a expressão de maneira conveniente, obtemos a forma desejada (3.1.5).

Procederemos agora à demonstração do Teorema 3.1.1.

Demonstração. (Teorema 3.1.1) Distinguimos dois casos, de acordo com a paridade de $\alpha$. Em ambos os casos, calcularemos o índice do campo vetorial na origem. Conforme apresentado no Exemplo 3.1.4, todo ponto crítico monodrômico tem índice +1 .

Assuma que $\alpha$ seja par. Fazendo uma reparametrização, podemos considerar $a=1$. Escolha a curva fechada simples $C_{\delta}=\left\{x^{2}+y^{2}=\delta^{2}\right\}$, com $\delta$ suficientemente pequeno, e a reta $L=\{x=0\}$. Aplicaremos a Proposição 3.1.3 para calcular o índice na origem.

Primeiramente, observamos que existem apenas dois pontos em $C_{\delta}$ com campo vetorial paralelo a $L, M_{1}=(\delta, 0)$ e $M_{2}=(-\delta, 0)$. De fato, para ser paralelo a $L$, o campo tem que assumir a forma $(0, k), k \neq 0$. Como $X_{1}(0,0)=0$, numa vizinhança suficientemente pequena da origem temos $\left(1+X_{1}(x, y)\right)>0$. Logo, para que $\dot{x}=0$ em (3.1.5), precisamos ter $y=0$. Isso implica que $\dot{y}=f(x) \not \equiv 0$. Assim, o campo vetorial associado ao sistema de equações diferenciais (3.1.5) será paralelo a $L$ se, e somente se, $y=0$.

Agora, vamos estudar o campo vetorial na vizinhança de $M_{1}$. Para $M_{2}$, o estudo é análogo. 
Podemos escrever os pontos da curva $C_{\delta}$, numa vizinhança de $M_{1}=(\delta, 0)$, como $M_{\varepsilon}=$ $\left(\delta-\varepsilon, \pm \sqrt{2 \delta \varepsilon-\varepsilon^{2}}\right), 0 \leq \varepsilon \leq \delta$. Como anteriormente, considerando-se uma vizinhança suficientemente pequena da origem, temos $\left(1+X_{1}(x, y)\right)>0$. Tomando-se os pontos de $C_{\delta}$ em que $y \geq 0$, conforme (3.1.5) temos $\dot{x} \geq 0$ e o sinal de $\dot{y}$ é determinado pelo sinal de $a x^{\alpha}$. Como $a=1>0$ e $\alpha$ é par, por hipótese, $\dot{y}>0$.

Assim, se tomarmos $0 \leq \varepsilon \leq \delta$ e $y \geq 0$, à medida que $\varepsilon$ diminui (isto é, percorremos a curva $C_{\delta}$ no sentido horário), o campo gira no sentido anti-horário, até que, para $\varepsilon=0$, temos o ponto $M_{1}=(\delta, 0)$, em que o campo é paralelo a $L$.

Analogamente, concluímos que o campo gira no sentido horário em torno de $M_{2}$. Portanto, o índice de (3.1.5) na origem é zero e neste caso, a origem não pode ser monodrômica.

Vamos agora assumir que $\alpha$ seja ímpar. Aplicando a Proposição 3.1.3 de modo análogo ao realizado anteriormente, tomando-se a mesma curva fechada simples $C_{\delta}$ e a direção $L$, concluímos que o índice da origem é $-\operatorname{sign}(a)$. De fato, para $a>0$, a situação é idêntica à anterior, porém o campo em $M_{2}=(-\delta, 0)$ terá sentido inverso, pois o sinal de $\dot{y}$ será determinado pelo sinal de $x$ (lembre que o sinal de $\dot{y}$ é determinado por $a x^{\alpha}$, mas neste caso, $a>0$ e $\alpha$ é ímpar), que numa vizinhança de $M_{2}$ é negativo. Assim, o índice do campo na origem neste caso será -1 .

Para $a<0$, temos $a x^{\alpha}<0$ para $x>0$ e $a x^{\alpha}>0$ para $x<0$. Como o sinal de $\dot{y}$ é determinado por $a x^{\alpha}$, não é difícil de verificar que em $M_{1}$ e $M_{2}$ o campo gira no sentido horário. Logo, o índice é igual a +1 .

Assim, para $\alpha$ ímpar, o índice do campo na origem é - sign(a). Portanto, os únicos sistemas candidatos a ter a origem como um ponto crítico monodrômico são aqueles com $\alpha$ ímpar e $a<0$.

Como nem todos os pontos singulares nilpotentes com índice +1 são monodrômicos - por exemplo, campos vetoriais com singularidade nilpotente do tipo nó possuem índice +1 ( - cf. [22] p.116-117) - efetuamos a reparametrização:

$$
\left\{\begin{array}{l}
x=\left(-\frac{1}{a}\right)^{\frac{1}{2 n-2}} \bar{x}, \\
y=-\left(-\frac{1}{a}\right)^{\frac{1}{2 n-2}} \bar{y}
\end{array}\right.
$$


renomeando $\bar{x}=x$ e $\bar{y}=y$, obtemos exatamente o sistema (3.1.5), estudado no Lema 3.1.6. Considerando-se as possíveis relações entre $\beta$ e $n-1$, temos os seguintes casos:

Caso $\beta<\mathbf{n}-\mathbf{1}$. Nesta situação, usando as expressões dadas por (3.1.4) do Lema 3.1.5, temos:

$$
\begin{aligned}
& P_{1}(x, y)=b x^{\beta} y^{2} \\
& P_{2}(x, y)=\left(a_{0}+d_{10}\right) x^{2 n} y \\
& P_{3}(x, y)=\left(a_{1}+d_{20} x^{2 n+1} y+\mathcal{X}_{\{n=2\}} d_{01} x^{2 n-1} y^{2} ;\right. \\
& P_{4}(x, y)=\left(a_{2}+d_{30}\right) x^{2 n+2} y+\mathcal{X}_{\{n=2\}}\left[d 11 x^{2 n} y^{2}+e_{01} y^{4}\right]+\mathcal{X}_{\{n=3\}}\left[d_{01} x^{2 n-1} y\right] ; \\
& Q_{1}(x, y)=1+b x^{\beta+1} y, \\
& Q_{2}(x, y)=a_{0} x^{2 n+1}+\left(e_{00}-n d_{10}\right) x y^{2}, \\
& Q_{3}(x, y)=a_{1} x^{2 n+2}+\left(e_{10}-n d_{20}\right) x^{2} y^{2}-\mathcal{X}_{\{n=2\}} 2 d_{01} y^{3} .
\end{aligned}
$$

onde $P_{i}(x, y)=P_{i}(\operatorname{Cs}(\theta), \operatorname{Sn}(\theta))=P_{i}(\theta)$, o mesmo valendo para $Q_{i}$.

Assim, temos

$$
\tilde{X}=\tilde{X}(r, \theta)=\left\{\begin{array}{l}
\dot{r}=b \operatorname{Cs}^{\beta}(\theta) \operatorname{Sn}^{2}(\theta) r^{\beta+1}+O\left(\|r\|^{\beta+2}\right) \\
\dot{\theta}=b \operatorname{Cs}^{\beta+1}(\theta) \operatorname{Sn}(\theta) r^{\beta}+O\left(\|r\|^{\beta+1}\right)
\end{array}\right.
$$

Observe que, nas equações (3.1.4), $P_{2}, P_{3}, P_{4}$ são coeficientes de potências de $r$ com expoentes respectivamente $n+1, n+2, n+3$. Como por hipótese $\beta<n-1$, segue que $n+i>\beta+i, i=1,2,3$. Procedimento análogo foi usado para $Q_{i}, i=2,3$.

Dividindo-se ambas as equações por $r^{\beta}$ :

$$
\bar{X}=\left\{\begin{array}{l}
\dot{r}=b \operatorname{Cs}^{\beta}(\theta) \operatorname{Sn}^{2}(\theta) r+O\left(\|r\|^{2}\right) \\
\dot{\theta}=b \operatorname{Cs}^{\beta+1}(\theta) \operatorname{Sn}(\theta)+O\left(\|r\|^{1}\right)
\end{array}\right.
$$

Fazendo $r=0$, obtemos, então:

$$
\left\{\begin{array}{l}
\dot{r}=0, \\
\dot{\theta}=b \mathrm{Cs}^{\beta+1}(\theta) \operatorname{Sn}(\theta) .
\end{array}\right.
$$


concluímos que o sistema possui $\theta=\{0, T / 2, T, 3 T / 2\}$, onde $T$ é o período das funções $\operatorname{Cs}(\theta)$ e $\operatorname{Sn}(\theta)$, como direções características. Consequentemente, as trajetórias tendem a, ou se afastam da origem com direção definida e portanto, a singularidade não pode ser monodrômica.

Caso $\beta>\mathbf{n}-\mathbf{1}$ ou $\phi \equiv \mathbf{0}$. Aplicando as expressões dadas por (3.1.4) do Lema 3.1.5, obtemos:

$$
\left\{\begin{array}{l}
\dot{r}=P_{2}(\theta) r^{n+1}+O\left(\|r\|^{n+2}\right), \\
\dot{\theta}=r^{n-1}+Q_{2}(\theta) r^{n}+O\left(\|r\|^{n+1}\right)
\end{array}\right.
$$

Após divisão por $r^{n-1}$ :

$$
\left\{\begin{array}{l}
\dot{r}=P_{2}(\theta) r^{2}+O\left(\|r\|^{3}\right), \\
\dot{\theta}=1+Q_{2}(\theta) r+O\left(\|r\|^{n+1}\right)
\end{array}\right.
$$

Para $r=0$, o sistema não possui singularidades. Consequentemente, a origem pode ser um centro ou um nó.

Observamos que as expressões acima são válidas tanto para a condição $\beta>n-1$ quanto para $\phi \equiv 0$. Neste último caso, usando a expressão de $\phi(x)$ dada em (3.1.3), tomamos $b=b_{i}=0, \forall i>1$.

Caso $\beta=\mathbf{n}-\mathbf{1}$. Analogamente ao efetuado nos dois outros casos, aplicamos as expressões dadas por (3.1.4) do Lema 3.1.5 para obtermos:

$$
\left\{\begin{array}{l}
\dot{r}=r^{n}\left(b \mathrm{Cs}^{\beta}(\theta) \operatorname{Sn}^{2}(\theta)\right)+P_{2}(\theta) r^{n+1}+O\left(\|r\|^{n+2}\right), \\
\dot{\theta}=r^{n-1}\left(1+b \mathrm{Cs}^{\beta+1}(\theta) \operatorname{Sn}(\theta)\right)+Q_{2}(\theta) r^{n}+O\left(\|r\|^{n+1}\right)
\end{array}\right.
$$

Dividindo por $r^{n-1}$,obtemos:

$$
\left\{\begin{array}{l}
\dot{r}=r\left(b \operatorname{Cs}^{\beta}(\theta) \operatorname{Sn}^{2}(\theta)\right)+O\left(\|r\|^{2}\right) \\
\dot{\theta}=1+b \operatorname{Cs}^{\beta+1}(\theta) \operatorname{Sn}(\theta)+O(\|r\|)
\end{array}\right.
$$

Para $r=0$, considere a função $g(\theta)=1+b \operatorname{Cs}^{n}(\theta) \operatorname{Sn}(\theta)$.

Usando a Propriedade 1 da Proposição 1.4.12, podemos rescrever a função como $g(\theta)=$ $\operatorname{Cs}^{2 n}(\theta)+n \operatorname{Sn}^{2}(\theta)+b \operatorname{Cs}^{n}(\theta) \operatorname{Sn}(\theta)$. Fazendo a substituição:

$$
\left\{\begin{array}{l}
\operatorname{Cs}^{n}(\theta)=t \\
\operatorname{Sn}(\theta)=w
\end{array}\right.
$$


obtemos $g(t, w)=t^{2}+n w^{2}+b t w \stackrel{t \neq 0}{=} n\left(\frac{w}{t}\right)^{2}+b\left(\frac{w}{t}\right)+1$. Observe que, se $\operatorname{Cs}^{n}(\theta)=t=0$, então $g(\theta) \equiv 1 \neq 0$.

O discriminante desta função do segundo grau em $\frac{w}{t}$ é $\triangle=b^{2}-4 n$. Distinguimos então três possibilidades:

(i) Se $b^{2}-4 n<0$, a função $g(\theta)$ não se anula. Logo, usando argumento similar ao caso anterior $(\beta>n-1)$, concluímos que o sistema não tem direções características, isto é, a origem é monodrômica;

(ii) Se $b^{2}-4 n>0, g(\theta)$ apresenta quatro zeros simples, ou seja, admite separatrizes e consequentemente, não pode ser monodrômica .

(iii) Se $b^{2}-4 n=0$, então $g(\theta)$ não muda de sinal e possui duas raízes duplas. Essas raízes representam singularidades degeneradas do sistema e para determinar se neste caso há monodromia, efetuamos uma análise mais detalhada, a seguir.

Efetuando uma nova mudança de coordenadas $x=x, w=\frac{y}{x^{n}}$, o sistema resultante, após divisão por $x^{n-1}$ é:

$$
\left\{\begin{array}{l}
\dot{x}=x w(-1+O(\|(x, w)\|), \\
\dot{w}=1+b w+w^{2}+O(\|(x, w)\|) .
\end{array}\right.
$$

Observe que o ponto $(x, w)=\left(0, \frac{-b}{2 n}\right)$ é uma singularidade semi-hiperbólica deste sistema. Esta singularidade, de fato, é do tipo sela-nó. Em particular, para o sistema acima existem infinitas órbitas tendendo a esta singularidade. Em consequência, a origem não pode ser monodrômica.

Desta forma, concluímos que o único caso de monodromia, para $\beta=n-1$, ocorre sob a condição $b^{2}-4 n<0$.

\subsubsection{Constantes de Lyapunov generalizadas}

As constantes de Lyapunov generalizadas buscam estender a ideia empregada por Lyapunov na caracterização de centros em sistemas com singularidade não degeneradas ( - cf. [37]), para sistemas que possuem ponto singular isolado degenerado. Neste trabalho, vamos nos restringir ao estudo das constantes de Lyapunov generalizadas para pontos 
singulares nilpotentes. O caso de sistemas com pontos singulares linearmente nulos ainda é um problema em aberto, apesar de já existirem alguns resultados utilizando constantes de Lyapunov generalizadas em famílias particulares de sistemas (ver por exemplo [38, 24, 9]).

Na seção anterior, mostramos no Lema 3.1.5 que o sistema (3.1.3), em coordenadas polares, é expresso por (3.1.4). A demonstração do Teorema 3.1.1 nos assegura que, no caso monodrômico, a componente $\dot{\theta}$ em (3.1.4) não se anula em uma vizinhança do ponto singular, à exceção deste próprio ponto. Portanto, o sistema diferencial (3.1.4) pode ser escrito, nesta vizinhança da origem como:

$$
\frac{d r}{d \theta}=\sum_{i=1}^{\infty} R_{i}(\theta) r^{i}
$$

onde $R_{i}(\theta), i \geq 1$ são funções T-periódicas, T sendo o período das coordenadas $(1, n)$-quasehomogêneas (vide Definição 1.4 .10 e Eq. (1.4.7)).

Considere a solução da Eq. (3.1.7), tal que $r=\rho$ para $\theta=0$. Podemos escrevê-la como

$$
r(\theta, \rho)=\sum_{i=1}^{\infty} u_{i}(\theta) \rho^{i},
$$

$\operatorname{com} u_{1}(0)=1, u_{i}(0)=0, \forall i \geq 2$, pois $r(0, \rho)=\rho=\sum_{i=1}^{\infty} u_{i}(0) \rho^{i}$.

Consequentemente, a aplicação de primeiro retorno é dada pela série:

$$
\Pi(\rho)=\sum_{i=1}^{\infty} u_{i}(T) \rho^{i} .
$$

Para um dado sistema, o único termo significativo é o primeiro que faz com que a aplicação de primeiro retorno seja diferente da aplicação identidade. Este termo determinará a estabilidade do sistema. Por outro lado, se considerarmos uma família de sistemas com dependência em parâmetros, cada um dos $u_{i}(T)$ dependerá destes parâmetros.

Definição 3.1.7. Definimos a k-ésima constante de Lyapunov generalizada, denotada por $V_{k}$, como sendo o valor de $u_{k}(T)$, assumindo $u_{1}(T)=1$ e $u_{2}(T)=\ldots=u_{k-1}(T)=0$. Ou seja, $V_{k}:=u_{k}(T), u_{1}(T)=1$ e $u_{i}(T)=0, \forall i, 2 \leq i \leq m-1$.

Observe que o sistema (3.1.2) tem um centro na origem se, e somente se, $\Pi(\rho) \equiv \rho$, isto é, $V_{1}=1$ e $V_{k}=0, \forall k \geq 2$. 
As constantes de Lyapunov generalizadas também possibilitam investigar bifurcações de ciclos limites a partir da origem. Não abordaremos este estudo na dissertação.

\subsubsection{Problema da estabilidade}

Uma vez solucionado o problema da monodromia, precisamos investigar o problema da estabilidade, isto é, dado um sistema com singularidade monodrômica nilpotente isolada, desejamos determinar se esta singularidade é um foco ou um centro.

O problema da estabilidade foi resolvido por Moussu [41], em 1982. Usaremos uma abordagem distinta da originalmente adotada por Moussu, baseada em artigo de Gasull \& Álvarez [2], de 2006.

Seja um sistema diferencial planar analítico, com singularidade nilpotente $p$, que, sem perda de generalidade, assumiremos como sendo a origem (Lema 1.2.7). Pela Proposição 1.2.10, este sistema pode ser escrito na forma

$$
\left\{\begin{array}{l}
\dot{x}=-y+X_{2}(x, y), \\
\dot{y}=Y_{2}(x, y)
\end{array}\right.
$$

onde $X_{2}, Y_{2}$ são funções analíticas cujas séries de potências começam em termos de segunda ordem ou superior.

Takens [51] provou que um sistema com parte linear nilpotente, isto é, sistema da forma (3.1.9), pode ser formalmente transformado em um sistema Liénard generalizado:

$$
\left\{\begin{array}{l}
\dot{x}=-y, \\
\dot{y}=a(x)+y \tilde{b}(x),
\end{array}\right.
$$

onde $a(x)=a_{s-1} x^{s-1}(1+O(\|x\|)), s \geq 3$ e $\tilde{b}(x)$ com $\tilde{b}(0)=0$ são séries de potência formais. Além do artigo original de Takens [51], a demonstração deste resultado também pode ser encontrada em [8], p.82-83.

Em 2002, Stróżyna \& Żoładek [50] provaram que esta forma normal pode ser obtida por meio de mudança de coordenadas analítica. 
Lema 3.1.8. Numa vizinhança da origem e após uma mudança de coordenadas e reparametrização do tempo, o sistema (3.1.10)

$$
\left\{\begin{array}{l}
\dot{x}=-y \\
\dot{y}=a(x)+y \tilde{b}(x)
\end{array}\right.
$$

com $a(x)=x^{2 n-1}\left(a_{2 n-1}+O(\|x\|)\right), a_{2 n-1}>0$, pode ser transformado em:

$$
\left\{\begin{array}{l}
\dot{x}=-y \\
\dot{y}=x^{2 n-1}+b(x)
\end{array}\right.
$$

onde b(x) é uma função analítica fornecida na demonstração do lema.

Demonstração. Efetuamos as seguintes mudanças de coordenadas e reparametrização do tempo:

$$
\begin{aligned}
u & =\sqrt[2 n]{2 n \int_{0}^{x} a(s) d s}:=\Phi(x)=x \sqrt[2 n]{\left.a_{2 n-1}+O(\|x\|)\right)} \\
\frac{d t}{d t_{1}} & =\frac{u^{2 n-1}}{a(x)}=\frac{\left(a_{2 n-1} x^{2 n-1}+O\left(\|x\|^{2 n-1}\right)\right)^{(2 n-1) /(2 n)}}{a_{2 n-1} x^{2 n-1}+O\left(\|x\|^{2 n}\right)} \\
& =a_{2 n-1}^{-1 /(2 n)}+O(\|x\|)
\end{aligned}
$$

Temos então:

$$
\begin{aligned}
\frac{d u}{d t}=\dot{u} & =\frac{1}{2 n}\left(2 n \int_{0}^{x} a(s) d s\right)^{\frac{1}{2 n}-1} 2 n a(x) \dot{x} \\
& =\left[a(x)\left(2 n \int_{0}^{x} a(s) d s\right)^{\left(-\frac{2 n-1}{2 n}\right)}\right](-y) \\
& \stackrel{(3.1 .12)}{=} a(x) u^{-(2 n-1)}(-y) .
\end{aligned}
$$

Agora, $u^{\prime}=\frac{d u}{d t_{1}}=\frac{d u}{d t} \frac{d t}{d t_{1}}$.

Efetuando as substituições convenientes, obtemos $u^{\prime}=-y$. 
Analogamente, temos:

$$
\begin{aligned}
y^{\prime} & =\frac{d y}{d t_{1}}=\frac{d y}{d t} \frac{d t}{d t_{1}} \\
& =\frac{u^{2 n-1}}{a(x)}[a(x)+y \tilde{b}(x)] \\
& \stackrel{x=\Phi^{-1}(u)}{=} u^{2 n-1}+y\left[\frac{u^{2 n-1} \tilde{b}\left(\Phi^{-1}(u)\right)}{a\left(\Phi^{-1}(u)\right)}\right] .
\end{aligned}
$$

Ou seja,

$$
\left\{\begin{array}{l}
u^{\prime}=-y, \\
y^{\prime}=u^{2 n-1}+y b(u),
\end{array}\right.
$$

$\operatorname{com} b(u)=\frac{u^{2 n-1} \tilde{b}\left(\Phi^{-1}(u)\right)}{a\left(\Phi^{-1}(u)\right)}$ função analítica numa vizinhança da origem (composição de funções analíticas), como desejado.

Proposição 3.1.9 (Caracterização de Centros). Considere o sistema analítico

$$
\left\{\begin{array}{l}
\dot{x}=-y, \\
\dot{y}=x^{2 n-1}+y b(x),
\end{array}\right.
$$

com $b(x)=\sum_{j \geq \beta} b_{j} x^{j}$, e assuma que a origem é uma singularidade monodrômica deste sistema, isto é, uma das seguintes condições é satisfeita:

(i) $\beta>n-1$,

(ii) $\beta=n-1$ e $b_{\beta}^{2}-4 n<0$,

(iii) $b(x) \equiv 0$.

Então, a origem é um centro se, e somente se, $b^{e}(x):=\frac{b(x)+b(-x)}{2} \equiv 0$.

Demonstração. Do Teorema 3.1.3, segue que as condições de monodromia estão satisfeitas para o sistema (3.1.13). 
Suponha que $b^{e}(x) \equiv 0$. Aplicando a mudança de coordenadas $x=-\bar{x}$ e a reparametrização do tempo $t=-\bar{t}$, obtemos:

$$
\frac{d \bar{x}}{d \bar{t}}=\frac{d \bar{x}}{d t} \frac{d t}{d \bar{t}}=-\frac{d \bar{x}}{d t} \stackrel{x=-\bar{x}}{=} \frac{d x}{d t}=-y
$$

Analogamente,

$$
\frac{d y}{d \bar{t}}=\frac{d y}{d t} \frac{d t}{d \bar{t}}=-\frac{d y}{d t}
$$

Como $b^{e}(x) \equiv 0$ por hipótese, segue que $b(x)=-b(-x)=-b(\bar{x})$. Portanto,

$$
\left.-\frac{d y}{d \bar{t}}=(-\bar{x})^{2 n-1}+y(-b(\bar{x}))=-(\bar{x})^{2 n-1}-y b(\bar{x})\right)
$$

Logo, $\frac{d y}{d \bar{t}}=\bar{x}^{2 n-1}+y b(\bar{x})$. Ou seja, obtemos o mesmo sistema.

Usando o Critério de Reversibilidade de Poincaré ( - cf. [44], Teorema 6 p.145), concluímos que a origem do sistema (3.1.13) é um centro.

Reciprocamente, suponha que a origem seja um centro. A prova é feita por contradição.

Assuma que $b^{e}(x)=x^{2 l}\left(b_{2 l}+O(\|x\|)\right)$, para algum $b_{2 l} \neq 0$. Defina $b^{o}(x)=b(x)-b^{e}(x)$. Então, dado um sistema analítico (3.1.13), podemos construir, a partir deste sistema, o seguinte sistema

$$
\left\{\begin{array}{l}
\dot{x}=-y, \\
\dot{y}=x^{2 n-1}+y b^{o}(x) .
\end{array}\right.
$$

Observe que o sistema (3.1.14) possui $\left(b^{o}\right)^{e}(x) \equiv 0$ por construção. Já demonstramos que sistemas com tal condição possuem um centro na origem.

Lembremos que, se $p \in \mathbb{R}^{2}$ é um ponto singular de um sistema diferencial analítico planar, então $p$ é um centro se, e somente se, existe uma integral primeira $C^{\infty}$ definida em alguma vizinhança de $p$ - cf. [39]. Assim, se a origem de (3.1.14) é um centro, então existe uma função $V=V(x, y): U \subset \mathbb{R}^{2} \rightarrow \mathbb{R}, U$ uma vizinhança da origem, tal que $V(x, y)=k$, são curvas integrais de (3.1.14). Logo, $\dot{V}(x, y)=\frac{\partial V}{\partial x} \dot{x}+\frac{\partial V}{\partial y} \dot{y}$.

Portanto,

$$
\begin{aligned}
\dot{V}(x, y) & =\left(-x^{2 n-1}-y b^{o}(x)\right)(-y)+(-y)\left(x^{2 n-1}+y b(x)\right) \\
& =y^{2} x^{2 l}\left(b_{2 l}+O(\|x\|)\right)
\end{aligned}
$$


Deste modo, $\dot{V}$ não muda de sinal numa vizinhança da origem. No entanto, pelo Teorema de Lyapunov - cf. [52], a origem não pode ser um centro, contradizendo nossa hipótese. Logo, e segue o resultado.

Finalmente podemos enunciar o principal teorema desta seção.

Teorema 3.1.10 (Teorema da Estabilidade para Singularidades Nilpotentes e Cálculo das Constantes de Lyapunov Generalizadas). Seja um sistema analítico planar, com uma singularidade nilpotente monodrômica. Então, existe uma mudança de coordenadas analítica, tal que o sistema pode ser escrito como:

$$
\left\{\begin{array}{l}
\dot{x}=-y \\
\dot{y}=x^{2 n-1}+y b(x)
\end{array}\right.
$$

$\operatorname{com} b(x) \equiv 0$ ou $b(x)=\sum_{j \geq \beta} b_{j} x^{j}, b_{\beta} \neq 0$ e satisfazendo uma das seguintes condições:

(i) $\beta>n-1$,

(ii) $\beta=n-1$ e $b_{\beta}^{2}-4 n<0$.

Além disso:

1. Se $b(x)=b^{o}(x)+x^{2 l}\left(b_{2 l}+O(\|x\|)\right)$, com $b_{2 l} \neq 0$, e $b^{o}(x):=\frac{b(x)-b(-x)}{2}$, então $a$ primeira constante de Lyapunov generalizada significativa é:

(a) $V_{2-n+2 l}=K b_{2 l}$, onde ou $\beta>n-1$, ou $\beta=n-1$ e $\beta$ é ímpar. $K=K\left(n, l, b_{n-1}\right)$ é uma constante positiva fornecida na demonstração;

(b) $V_{1}=\exp \frac{2 b_{\beta} \pi}{n \sqrt{4 n-b_{\beta}^{2}}}$, onde $\beta=2 l=n-1$.

2. A origem é um centro se, e somente se, $b^{e}(x):=b(x)-b^{o}(x) \equiv 0$.

Para provar o Teorema 3.1.10, apresentamos um importante resultado, inspirado em $[19,54]$. Este resultado fornece uma maneira de computar a primeira constante de Lyapunov significativa para um sistema definido em um cilindro, aproveitando-se de um centro "nas proximidades" do referido sistema. 
Proposição 3.1.11. Seja uma equação diferencial ordinária analítica, da forma

$$
\frac{d r}{d \theta}=S(r, \theta)+r^{m}(v(\theta)+O(\|r\|)), \quad m \geq 1
$$

definida em um cilindro $(r, \theta) \in \mathbb{R} \times \mathbb{R} /[0, T]$ e assuma que a equação

$$
\frac{d r}{d \theta}=S(r, \theta)=r\left(S_{1}(\theta)+O(\|r\|)\right)
$$

é tal que $r=0$ é uma solução periódica, e além disso, que todas as soluções nas vizinhanças desta solução também são periódicas ( $r=0$ é um centro).

Então:

(a) se $m=1$, então $V_{1}=\exp \left(\int_{0}^{T} v(\theta) d \theta\right)$;

(b) se $m>1$, então

(b.1) $V_{1}=1$;

(b.2) $V_{i}=0, \forall i=2, \ldots, m-1 ; e$

(b.3) $V_{m}=\int_{0}^{T} v(\theta) e^{(m-1) \int_{0}^{\theta} S_{1}(\psi) d \psi} d \theta$.

Demonstração. Por meio de procedimento similar ao realizado em [19, 54], expressamos (3.1.16) em um novo sistema de coordenadas $(R, \theta)$, onde $R=F(r, \theta)$ uma mudança de coordenadas tal que $\frac{d R}{d \theta} \equiv 0$. Vamos construir esta mudança de coordenadas.

A ideia geométrica é associar a cada ponto da órbita periódica de (3.1.17), o valor da intersecção da órbita com a reta $\theta=0$. Mais concretamente, seja $\varphi\left(\theta ;\left(\theta_{0}, r_{0}\right)\right)$ a solução de (3.1.17), tal que $r=r_{0}$, quando $\theta=\theta_{0}$. Então, definimos:

$$
R=F(\theta, r):=\varphi(-\theta ;(\theta, r))
$$

a Eq. (3.1.17) se escreve como $\frac{d R}{d \theta}=0$.

A inversa da mudança acima é dada por:

$$
r=G(\theta ; R):=\varphi(\theta ;(0, R)),
$$


que toma um ponto na reta $\theta=0$ de coordenadas $(0, R)$, e leva ao ponto $\varphi(\theta ; \varphi(\theta ;(0, R)))$, da órbita periódica $\varphi$ de (3.1.17). Escrevendo

$$
\varphi(\theta ;(0, R))=R\left(u_{1}(\theta)+O(\|R\|)\right)
$$

obtemos

$$
u_{1}(\theta)=\exp \left(\int_{0}^{\theta} S_{1}(\psi) d \psi\right)
$$

De fato, como $\varphi$ é solução da Eq. (3.1.17), temos:

$$
\frac{d}{d \theta}(\varphi(\theta ;(0, R)))=\varphi(\theta ;(0, R))\left[S_{1}(\theta)+O(\|\varphi(\theta ;(0, R))\|)\right]
$$

que pode ser reescrita, por (3.1.20), como

$$
\frac{d}{d \theta}\left(R\left(u_{1}(\theta)+O(\|R\|)\right)=R\left(u_{1}(\theta)+O(\|R\|)\left[S_{1}(\theta)+O(\|\varphi(\theta ;(0, R))\|]\right.\right.\right.
$$

Agora, efetuando a Regra da Cadeia no primeiro membro da equação acima e usando o fato que $\frac{d R}{d \theta} \equiv 0$, obtemos, com mais algumas operações algébricas simples no segundo membro, o seguinte resultado:

$$
\frac{d}{d \theta} u_{1}(\theta)=u_{1}(\theta) s_{1}(\theta)
$$

e integrando a expressão acima, obtemos (3.1.21).

A expressão (3.1.21) prova que (3.1.18) e (3.1.19) são de mudanças de coordenadas, e também que

$$
\begin{aligned}
r & =G(\theta, R)=R\left(u_{1}(\theta)+O(\|R\|)\right. \\
R & =F(\theta, r)=r\left(\frac{1}{u_{1}(\theta)}+O(\|r\|)\right)
\end{aligned}
$$

Das relações acima, temos:

$$
\frac{\partial F(\theta, r)}{\partial \theta}=-\frac{r}{u_{1}(\theta)} S_{1}(\theta)=-\left(\frac{1}{u_{1}(\theta)}+O(\|r\|)\right) r S_{1}(\theta)=-\frac{\partial F(\theta, r)}{\partial r} S(r, \theta) .
$$


Vamos escrever Eq. (3.1.16) neste novo sistema de coordenadas:

$$
\begin{aligned}
\frac{d R}{d \theta} & =\frac{\partial F(\theta, r)}{\partial \theta}+\frac{\partial F(\theta, r)}{\partial r} \frac{d r}{d \theta} \\
& =\frac{\partial F(\theta, r)}{\partial \theta}+\frac{\partial F(\theta, r)}{\partial r}\left[S(r, \theta)+r^{m}(v(\theta)+O(\|r\|))\right] \\
& \left.=\frac{\partial F(\theta, r)}{\partial \theta}+\frac{\partial F(\theta, r)}{\partial r} S(r, \theta)+\frac{\partial F(\theta, r)}{\partial r} r^{m}(v(\theta)+O(\|r\|))\right] \\
& \stackrel{(3.1 .22)}{=} \frac{\partial F(\theta, r)}{\partial \theta}\left[r^{m}(v(\theta)+O(\|r\|))\right]=r^{m}\left(\frac{v(\theta)}{u_{1}(\theta)}+O(\|r\|)\right) \\
& =R^{m}\left(u_{1}^{m-1}(\theta) v(\theta)+O(\|R\|)\right) .
\end{aligned}
$$

Como em $\theta=0$ as duas coordenadas $r$ e $R$ coincidem, nós calculamos a aplicação de Poincaré de $\theta=0$ até $\theta=T$ na última variável. Agora, escrevendo a solução começando em $R=r=\rho$ quando $\theta=0$, como

$$
R(\theta, \rho)=\sum_{i \geq 1} w_{i}(\theta) \rho^{i}
$$

$\operatorname{com} w_{1}(0)=1$ e $w_{i}(0)=0, \forall i \geq 1$, pois $R(0, \rho)=\rho=\sum_{i \geq 1} w_{i}(0) \rho^{i}$

Se $m=1$, pelas Eqs. (3.1.23) e (3.1.24) temos $\frac{\partial R}{\partial \theta}=R(v(\theta)+O(\|R\|))=$ $=\sum_{i \geq 1} w_{i}(\theta) \rho^{i}(v(\theta)+O(\|R\|))$. Por outro lado, derivando a Eq. (3.1.24), temos $\frac{\partial R}{\partial \theta}=$ $\sum_{i \geq 1} w_{i}^{\prime}(\theta) \rho^{i}, w_{i}^{\prime}=\frac{d w_{i}}{d \theta}$. Igualando estas duas expressões segue que $\sum_{i \geq 1} w_{i}^{\prime}(\theta) \rho^{i}=\sum_{i \geq 1} w_{i}(\theta) \rho^{i}(v(\theta)+$ $O(\|R\|))$. Daí, tomando $i=1$ resulta em $w_{1}^{\prime}(\theta)=w_{1}(\theta) v(\theta)$ e então $w_{1}(\theta)$ pode ser facilmente obtida.

Se $m>1$, então efetuando-se operações análogas àquelas do caso anterior temos $\sum_{i \geq 1} w_{i}^{\prime}(\theta) \rho^{i}=\left(\sum_{i \geq 1} w_{i}(\theta) \rho^{i}\right)^{m}\left(u_{1}^{m-1}(\theta) v(\theta)+O(\|R\|)\right)$ e portanto, segue que $w_{i}^{\prime}(\theta)=0, \forall i=$ $1, \ldots, m-1$. Como $w_{1}(0)=1$ e $w_{i}(0)=0, \forall i \geq 1$, seque que $w_{1}(\theta)=w_{1}(0)=1 \mathrm{e}$ $w_{i}(\theta)=w_{i}(0)=0, \forall i=1, \ldots, m-1$.

Para $i=m, w_{m}^{\prime}(\theta)=w_{m}(\theta) u_{1}^{m-1}(\theta) v(\theta)$. Logo,

$$
w_{m}(\theta)=\int_{0}^{\theta} u_{1}^{m-1}(\psi) d(\psi)
$$

Como $V_{m}=w_{m}(T)$, o resultado segue. 
Demonstração. (Teorema 3.1.10) Conforme já comentado no início desta seção, a existência de uma mudança de coordenadas analítica que possibilita escrever um sistema analítico planar, com uma singularidade nilpotente monodrômica, na forma (3.1.15) é provada em [50].

Para dar a expressão das constantes de Lyapunov generalizadas da forma normal (3.1.15), vamos escrever este sistema usando coordenadas $(1, n)$-quasehomogêneas $(x, y)$

$$
\left\{\begin{array}{l}
x=r \operatorname{Cs}(\theta), \\
y=r^{n} \operatorname{Sn}(\theta) .
\end{array}\right.
$$

Lembramos que um sistema diferencial analítico planar da forma

$$
\left\{\begin{array}{l}
\dot{x}=X(x, y) \\
\dot{y}=Y(x, y)
\end{array}\right.
$$

se escreve, em coordenadas (1,n)-quasehomogêneas como

$$
\left\{\begin{array}{l}
\dot{r}=\frac{x^{2 n-1} X(x, y)+y Y(x, y)}{r^{2 n-1}}, \\
\dot{\theta}=\frac{x Y(x, y)-n y X(x, y)}{r^{n+1}} .
\end{array}\right.
$$

conforme apresentado pela expressão (1.4.8), na Seção 3.1.1.

Para o sistema (3.1.15), temos

$$
\dot{r}=\frac{(r \operatorname{Cs} \theta)^{2 n-1}\left(-r^{n} \operatorname{Sn} \theta\right)+\left(r^{n} \operatorname{Sn} \theta\right)\left[(r \operatorname{Cs} \theta)^{2 n-1}+\left(r^{n} \operatorname{Sn} \theta\right)+\sum_{j \geq \beta} b_{j}\left(r^{n} \operatorname{Cs} \theta\right)^{j}\right]}{r^{2 n-1}}
$$




$$
\begin{aligned}
\dot{\theta} & =\frac{(r \operatorname{Cs} \theta)\left[(r \operatorname{Cs} \theta)^{2 n-1}+\left(r^{n} \operatorname{Sn} \theta\right) \sum_{j \geq \beta} b_{j}\left(r^{n} \operatorname{Cs} \theta\right)^{j}\right]-n\left(r^{n} \operatorname{Sn} \theta\right)\left(-r^{n} \operatorname{Sn} \theta\right)}{r^{n+1}} \\
& =r^{n-1} \operatorname{Cs}^{2 n} \theta+\operatorname{Sn} \theta \operatorname{Cs} \theta \sum_{j \geq \beta} b_{j}\left(r^{n} \operatorname{Cs} \theta\right)^{j}+n r^{n-1} \operatorname{Sn}^{2} \theta \\
& =r^{n-1}\left(\operatorname{Cs}^{2 n} \theta+n \operatorname{Sn}^{2} \theta\right)+\sum_{j \geq \beta} b_{j} r^{j} \operatorname{Sn} \theta \operatorname{Cs}^{j+1} \theta \stackrel{\text { Prop.3.1.22 }}{=} r^{n-1}+\sum_{j \geq \beta} b_{j} r^{j} \operatorname{Sn} \theta \mathrm{Cs}^{j+1} \theta .
\end{aligned}
$$

$\operatorname{Assim}, \frac{d r}{d \theta}=\frac{\sum_{j \geq \beta} b_{j} \operatorname{Sn}^{2} \theta \mathrm{Cs}^{j} \theta r^{j+1}}{r^{n-1}+\sum_{j \geq \beta} b_{j} r^{j} \operatorname{Sn} \theta \mathrm{Cs}^{j+1} \theta}$.

Dividindo o numerador e o denominador por $r^{n-1}$, temos então:

$$
\frac{d r}{d \theta}:=T(r, \theta)=\frac{\sum_{j \geq \beta} b_{j} \operatorname{Sn}^{2} \theta \mathrm{Cs}^{j} \theta r^{2-n+j}}{1+\sum_{j \geq \beta} b_{j} \operatorname{Sn} \theta \mathrm{Cs}^{j+1} \theta} r^{1-n+j} .
$$

Considere agora, a nova equação diferencial

$$
\frac{d r}{d \theta}:=S(r, \theta)=\frac{\sum_{j \geq \beta, \mathrm{j} \text { impar }} b_{j} \operatorname{Sn}^{2} \theta \mathrm{Cs}^{j} \theta r^{2-n+j}}{1+\sum_{j \geq \beta, \mathrm{j} \text { ímpar }} b_{j} \operatorname{Sn} \theta \mathrm{Cs}^{j+1} \theta} r^{1-n+j} .
$$

Observe que (3.1.26) é expressão de algum sistema da forma (3.1.13), em coordenadas polares generalizadas, em que todas as potências de $x$ em $b(x)$ possuem grau ímpar, por construção. Assim, pela Proposição 3.1.9, segue que a origem é um centro para Eq. (3.1.26).

Para aplicarmos a Proposição 3.1.11, vamos calcular $T(r, \theta)-S(r, \theta)$. Lembramos que, por hipótese, $b_{2 l} \neq 0$ e vale uma das condições, $\beta>n-1$ ou $\beta=n-1$. Como $2 l \geq \beta$, segue que $2 l \geq n-1$. Então

$$
T(r, \theta)-S(r, \theta)=b_{2 l} r^{2-n+2 l}(\bar{v}(\theta)+O(\|r\|)),
$$


onde

$$
\bar{v}(\theta)= \begin{cases}\operatorname{Sn}^{2} \theta \mathrm{Cs}^{2 l} \theta, & \text { se } \beta>n-1, \\ \frac{\operatorname{Sn}^{2} \theta \mathrm{Cs}^{2 l} \theta}{\left(1+b_{n-1} \operatorname{Sn} \theta \operatorname{Cs}^{n} \theta\right)^{2}}, & \text { se } \beta=n-1, \text { e } \beta \text { é ímpar, } \\ \frac{\operatorname{Sn}^{2} \theta \mathrm{Cs}^{2 l} \theta}{1+b_{n-1} \operatorname{Sn} \theta \mathrm{Cs}^{n} \theta}, & \text { se } \beta=2 l=n-1 .\end{cases}
$$

De fato, considere as expressões de $T(r, \theta)$ e $S(r, \theta)$ dadas, respectivamente, pelas Eqs. (3.1.25) e (3.1.26). Considere ainda, a aproximação $(1+O(\|r\|))^{-1} \simeq(1-O(\|r\|))$, válida numa vizinhança suficientemente pequena da origem. Vamos então, analisar separadamente cada um dos casos.

Caso 1: $\beta>\mathbf{n}-\mathbf{1}$. Em ambos os denominadores das expressões (3.1.25) e (3.1.26), o menor grau em $r$ é $1-n+\beta=\beta-(n-1) \geq 1$. Assim, utilizando a aproximação mencionada acima, podemos considerar os denominadores de $T(r, \theta)$ e $S(r, \theta)$ como unitários, numa vizinhança suficientemente pequena da origem. Como os índices de $b_{i}$ de $S(r, \theta)$ são todos ímpares, então a diferença $T(r, \theta)-S(r, \theta)=\sum_{j \geq 2 l, \mathrm{j} \text { par }} b_{j} \operatorname{Sn}^{2} \theta \mathrm{Cs}^{j} \theta r^{2-n+j}=$ $b_{2 l} r^{2-n+2 l}\left(\operatorname{Sn}^{2} \theta \mathrm{Cs}^{2 l} \theta+O(\|r\|)\right)$.

Caso 2: $\beta=\mathbf{n}-1$ e $\beta$ ímpar. Em ambos os denominadores das expressões de $T(r, \theta)$ e $S(r, \theta)$, o menor grau em $r$ é $1-n+\beta=\beta-(n-1)=0$. Logo, os denominadores de (3.1.25) e (3.1.26) são, respectivamente, da forma $\left[1+b_{n-1} \operatorname{Sn} \theta \operatorname{Cs}^{n} \theta+b_{n} \operatorname{Sn} \theta \operatorname{Cs}^{n+1} \theta r+\right.$ ... e $\left[1+b_{n-1} \operatorname{Sn} \theta \operatorname{Cs}^{n} \theta+b_{n+1} \operatorname{Sn} \theta \operatorname{Cs}^{n+2} \theta r^{2}+\ldots\right]$. Para computar $T(r, \theta)-S(r, \theta)$, precisamos calcular o mínimo múltiplo comum entre essas expressões. Efetuando-se um cálculo simples, obtemos $\left(1+b_{n-1} \operatorname{Sn} \theta \mathrm{Cs}^{n} \theta\right)^{2}+O(\|r\|)$, que pode ser aproximado para $(1+$ $\left.b_{n-1} \operatorname{Sn} \theta \operatorname{Cs}^{n} \theta\right)^{2}$ numa vizinhança suficientemente pequena da origem. Para o numerador, o cálculo é similar.

Caso 3: $\beta=2 \mathbf{l}=\mathbf{n}-\mathbf{1}$. O procedimento é análogo ao realizado nos dois casos anteriores, observando-se que neste caso, como $\beta$ é par, o denominador de $S(r, \theta)$ não possui termo da forma $b_{n-1} \operatorname{Sn} \theta \operatorname{Cs}^{n} \theta$.

Precisamos ainda, calcular, em cada um dos casos acima, a expressão $S(r, \theta)=$ $r\left(S_{1}(\theta)+O(\|r\|)\right)$. Nos Casos 1 e 3, $S_{1}(\theta) \equiv 0$. De fato, da Eq. (3.1.26) e usando a aproximação $(1+O(\|r\|))^{-1} \simeq(1-O(\|r\|))$, segue que o menor grau de $r$ em $S(r, \theta)$ é 
$k=j-(n-2)$, com $j=\beta$, se $\beta$ for ímpar, ou $j=\beta+1$, se $\beta$ for par. No Caso 1 , temos $\beta>n-1$. Daí, $k=j-(n-2) \stackrel{\beta>n-1}{\geq} 2$ e consequentemente, $S(r, \theta)$ não possui termos lineares em $r$. Para o Caso 3, $\beta$ é par, o que implica $k=j-(n-2)=\beta+1-(n-2) \stackrel{\beta=n-1}{\geq} 2$ e assim, tal como no primeiro caso, $S(r, \theta)$ não possui termos lineares em $r$.

No Caso 2 , como $\beta=n-1$ é impar, o grau da variável $r$ no primeiro termo de $S(r, \theta)$ é $k=\beta-(n-2)=1$. Assim,

$$
S_{1}(\theta)=\frac{b_{n-1} \operatorname{Sn}^{2} \theta \mathrm{Cs}^{n-1} \theta}{1+b_{n-1} \operatorname{Sn} \theta \mathrm{Cs}^{n} \theta}
$$

Finalmente temos todos os requisitos para aplicar a Proposição 3.1.11.

Se $\beta=2 l=n-1$ (Caso 2), então da Eq. (3.1.27) segue que $m=2-n+2 l=1$ e portanto,

$$
V_{1}=\exp \left[\frac{\operatorname{Sn}^{2} \theta \mathrm{Cs}^{2 l} \theta}{1+b_{n-1} \operatorname{Sn} \theta \mathrm{Cs}^{n} \theta}\right]
$$

Nos Casos 1 e $3,2 l>n-1$ e portanto, da Eq. (3.1.27), segue que $m=2-n+2 l>1$. Consequemente $V_{1}=1, V_{2}=\ldots=V_{1-n+2 l}=0$ e $V 2-n+2 l=b_{2 l} \int_{0}^{T} w(\theta) d \theta$, onde

$$
w(\theta)= \begin{cases}\operatorname{Sn}^{2} \theta \mathrm{Cs}^{2 l} \theta, & \text { se } \beta>n-1, \\ \frac{\operatorname{Sn}^{2} \theta \mathrm{Cs}^{2 l} \theta \exp \left[(1-n+2 l) \int_{0}^{\theta} S_{1}(\psi) d \psi\right]}{\left(1+b_{n-1} \operatorname{Sn} \theta \mathrm{Cs}^{n} \theta\right)^{2}}, & \text { se } \beta=n-1, \text { e } \beta \text { é ímpar }\end{cases}
$$

onde $S_{1}(\psi)$ é dada em (3.1.28).

As expressões acima fornecem a demonstração para o item (1) do Teorema 3.1.10, pois $w(\theta)$ é de fato, uma função não negativa (composição de funções quadráticas e exponencial).

Para finalizar a demonstração, calculamos explicitamente as integrais acima, em dois dos três casos.

Para computar a expressão (3.1.29), introduzimos a variável $x=\frac{\operatorname{Sn} \theta}{\operatorname{Cs}^{n} \theta}$. Segue que:

$$
\frac{\operatorname{Sn}^{2} \theta \mathrm{Cs}^{2 l} \theta}{1+b_{n-1} \operatorname{Sn} \theta \mathrm{Cs}^{n} \theta}=\frac{b_{n-1} x^{2} \mathrm{Cs}^{2 n} \theta \mathrm{Cs}^{n-1} \theta}{\left(\frac{1}{\mathrm{Cs}^{2 n}}+b_{n-1} x\right) \mathrm{Cs}^{2 n} \theta} \stackrel{\left(\mathrm{Cs}^{2 n} \theta=\frac{1}{\theta+n x^{2}}\right)}{=} \frac{b_{n-1} x^{2} \mathrm{Cs}^{3 n-1} \theta}{\left(1+b_{n-1} x+n x^{2}\right) \mathrm{Cs}^{2 n} \theta} .
$$


Além disso,

$$
\begin{aligned}
d x & \stackrel{\text { Def.1.4.6 }}{=} \frac{\mathrm{Cs}^{2 n-1} \theta \mathrm{Cs}^{n} \theta-\operatorname{Sn} \theta n \mathrm{Cs}^{n-1} \theta(-\operatorname{Sn} \theta)}{\mathrm{Cs}^{2 n} \theta} d \theta \\
& =\frac{\mathrm{Cs}^{n-1} \theta\left(\mathrm{Cs}^{2 n} \theta+n \operatorname{Sn}^{2} \theta\right)}{\mathrm{Cs}^{2 n} \theta} d \theta \\
& =\frac{1}{\mathrm{Cs}^{n+1} \theta} d \theta
\end{aligned}
$$

isto é, $d \theta=\mathrm{Cs}^{n+1} \theta d x$.

Substituindo as expressões acima em (3.1.29), obtemos

$$
\begin{aligned}
V_{1} & =2 \int_{-\infty}^{+\infty} \frac{b_{n-1} x^{2} \mathrm{Cs}^{3 n-1} \theta}{\left(1+b_{n-1} x+n x^{2}\right) \mathrm{Cs}^{2 n} \theta} \mathrm{Cs}^{n+1} \theta d x \\
& =\int_{-\infty}^{+\infty} \frac{2 b_{n-1} x^{2}}{\left(1+b_{n-1} x+n x^{2}\right)} \mathrm{Cs}^{2 n} \theta d x \\
& =\int_{-\infty}^{+\infty} \frac{2 b_{n-1} x^{2}}{\left(1+b_{n-1} x+n x^{2}\right)\left(1+n x^{2}\right)} d x \\
& =\exp \left(\frac{2 b_{n-1} \pi}{n \sqrt{4 n-b_{n-1}^{2}}}\right) .
\end{aligned}
$$

Quando $\beta>n-1$, obtemos

$$
V_{2-n+2 l}=b_{2 l} \int_{0}^{T} \operatorname{Sn}^{2} \theta \operatorname{Cs}^{2 l} \theta d \theta=\frac{\sqrt{\pi} \Gamma\left(\frac{2 l+1}{2 n}\right)}{\sqrt{n^{3}} \Gamma\left(\frac{3 n+2 l+1}{2 n}\right)} b_{2 l},
$$

onde usamos a Proposição 1.4.12.

A demonstração da afirmação (2) do Teorema 3.1.10 é resultado direto da Proposição 3.1 .9 .

Essencialmente, o método apresentado neste capítulo faz uso da teoria das formas normais para computar as constantes de Lyapunov generalizadas, que por sua vez são utilizadas para resolução do problema do centro-foco para singularidades nilpotentes. Apesar de interessante, esta abordagem é computacionalmente dispendiosa, conforme ilustraremos em detalhes, no Capítulo 4.

Apresentamos a seguir uma outra técnica, que investiga o problema do centro-foco para singularidades nilpotentes utilizando o método de Lyapunov para caracterização de centros em singularidades não degeneradas. 


\subsection{Método de Giacomini-Giné-Llibre}

Seja $p \in \mathbb{R}^{2}$ um ponto singular de um sistema diferencial em $\mathbb{R}^{2}$. Se $p$ é um centro, um problema interessante consiste em verificar a existência de uma integral primeira $H$ definida em alguma vizinhança $U$ de $p$ (i.e. uma função não constante $H: U \rightarrow \mathbb{R}$ tal que $H$ é constante sobre as órbitas do sistema diferencial).

Mais especificamente, vamos assumir um sistema diferencial analítico com um centro em $p$. Então, sabe-se que existe uma integral primeira $C^{\infty}$ definida em alguma vizinhança de $p$, ver [39]. Também é conhecido que existe uma primeira integral analítica definida em $U \backslash\{p\}$, para alguma vizinhança $U$ de $p$, ver [35]. Mas, em geral, essa integral primeira analítica não pode ser estendida a $p$. De fato, fixado um sistema diferencial analítico em $\mathbb{R}^{2}$ com um centro em $p$, ainda é um problema em aberto a caracterização da existência de uma integral primeira analítica numa vizinhança de $p$, ou como usualmente dizemos, uma integral primeira analítica local em $p$ - cf. [27].

Seja $p \in \mathbb{R}^{2}$ um ponto singular de um sistema diferencial analítico em $\mathbb{R}^{2}$, e assuma que $p$ é um centro. Sem perda de generalidade, podemos assumir que $p$ é a origem do sistema de coordenadas. Então, pelas Proposições 1.2.8, 1.2.10 e 1.2.11, o sistema pode ser escrito em uma das três formas seguintes:

$$
\begin{array}{ll}
\dot{x}=-y+F_{1}(x, y), & \dot{y}=x+F_{2}(x, y) ; \\
\dot{x}=y+F_{1}(x, y), & \dot{y}=F_{2}(x, y) ; \\
\dot{x}=F_{1}(x, y), & \dot{y}=F_{2}(x, y) ;
\end{array}
$$

onde $F_{1}(x, y)$ e $F_{2}(x, y)$ são funções reais analíticas sem termos constantes e lineares, definidas numa vizinhança da origem.

No que se segue, um centro de um sistema diferencial analítico em $\mathbb{R}^{2}$ é chamado de linear, nilpotente ou degenerado se, após uma mudança de coordenadas afim e um reescalonamento de tempo, puder ser escrito na forma (3.2.1), (3.2.2) ou (3.2.3), respectivamente.

O estudo dos centros lineares, em termos da existência de uma integral primeira analítica, é devido a Poincaré [45] e Lyapunov [36], ver também Moussu [42]. 
Teorema 3.2.1 (Teorema do Centro Linear). O sistema diferencial analítico real (3.2.1) tem um centro na origem se, e somente se, existe uma integral primeira analítica local da forma $H=x^{2}+y^{2}+F(x, y)$, definida numa vizinhança da origem, onde $F$ possui termos de grau maior ou igual a 2 .

O Algoritmo de Lyapunov - cf. 2.1.3, consiste justamente em determinar quando um sistema da forma (3.2.1) tem uma integral primeira analítica local na origem, e consequentemente, um centro neste ponto.

Para centros nilpotentes, apresentamos o teorema a seguir, em que o Algoritmo de Lyapunov é utilizado para investigar o problema do centro-foco.

Teorema 3.2.2 (Teorema do Centro Nilpotente). Suponha que a origem do sistema diferencial analítico (3.2.2) seja um centro. Então, existem funções analíticas $G_{1}$ e $G_{2}$, cujas expressões são fornecidas na demonstração, tais que o sistema

$$
\dot{x}=y+F_{1}(x, y)+\varepsilon G_{1}(x, y), \quad \dot{y}=-\varepsilon x+F_{2}(x, y)+\varepsilon G_{2}(x, y)
$$

tem um centro linear na origem, para todo $\varepsilon>0$.

Para demonstrarmos este teorema, vamos precisar de alguns resultados preliminares, que apresentamos a seguir.

A caracterização dos centros nilpotentes em termos da existência de uma simetria é devida a Berthier e Moussu - cf. [11] (ver também [50]), que obtiveram o seguinte teorema:

Teorema 3.2.3. Se o sistema analítico (3.2.2) tem um centro na origem, então existe uma mudança de coordenadas analítica, tal que, o novo sistema também apresenta a forma (3.2.2) e é invariante pela mudança de coordenadas $(x, y, t) \rightarrow(-x, y-t)$.

De [15], se o sistema analítico (3.2.2) tem um centro na origem e existe uma mudança de coordenadas analítica tal que o novo sistema também apresenta a forma (3.2.2) e é invariante pela mudança de coordenadas $(x, y, t) \rightarrow(-x, y-t)$, então o sistema tem uma primeira integral analítica definida numa vizinhança da origem.

Também precisaremos do próximo teorema, extraído de [44] p. 145: 
Teorema 3.2.4. Seja $E \subset \mathbb{R}^{2}$ aberto contendo a origem, e seja $f \in C^{1}(E), f(0)=0$. Se o sistema não linear $\dot{x}=f(x)$ é simétrico com respeito ao eixo $x$ ou ao eixo $y$, e se a origem for um centro para a parte linear do sistema, então a origem de $\dot{x}=f(x)$ é um centro.

Demonstração. (Teorema 3.2.2) Assuma que a origem do sistema (3.2.2) é um centro. O Teorema 3.2.3 e sua demonstração nos garantem que, para todo centro nilpotente (3.2.2), correspondendo a um campo vetorial analítico $X(x, y)$, existe uma mudança de coordenadas analítica $(x, y) \rightarrow(u, v)$ da forma

$$
x=u+\ldots \quad y=v+\ldots
$$

tal que $X(x, y)$ escrito nestas novas variáveis é um campo vetorial da forma

$$
Y(u, v)=\left(v+\bar{F}_{1}(u, v), \bar{F}_{2}(u, v)\right)
$$

onde $\bar{F}_{1}(u, v)$ e $\bar{F}_{2}(u, v)$ são funções analíticas com termos de segundo grau em $u$ e $v$ e o sistema diferencial associado é invariante sob a mudança de coordenadas $(x, y, t) \rightarrow$ $(-x, y-t)$.

Considere agora a seguinte perturbação do campo vetorial (3.2.6):

$$
Y_{\varepsilon}(u, v)=\left(v+\bar{F}_{1}(u, v),-\varepsilon u+\bar{F}_{2}(u, v)\right)
$$

$\operatorname{com} \varepsilon>0$. Então,

$$
D J Y_{\varepsilon}(0,0)=\left(\begin{array}{cc}
0 & 1 \\
-\varepsilon & 0
\end{array}\right)=\varepsilon>0
$$

e os autovalores da origem são $\pm \sqrt{\varepsilon} i$. Consequentemente, a origem é um centro para a parte linear de (3.2.7). Disto, e do fato de que o sistema diferencial associado ao campo vetorial (3.2.7) é invariante sob a mudança de coordenadas $(x, y, t) \rightarrow(-x, y-t)$ (pois o sistema não perturbado é invariante), segue do Teorema 3.2.4 que a origem do campo vetorial (3.2.7) é um centro linear, $\forall \varepsilon>0$.

Observe que, a mudança de coordenadas (3.2.5) preserva a parte linear do campo, pois seu jacobiano é da forma $\left(\begin{array}{ll}1 & 0 \\ 0 & 1\end{array}\right)$. Assim, a mudança de coordenadas inversa de (3.2.5) 
pode ser escrita como:

$$
\left\{\begin{array}{l}
u=x+f_{1}(x, y) \\
v=y+f_{2}(x, y)
\end{array}\right.
$$

onde $f_{1}(x, y), f_{2}(x, y)$ são funções analíticas sem termos constantes ou lineares. Diferenciando (3.2.8), obtemos

$$
\begin{aligned}
& \dot{u}=\left(1+\frac{\partial f_{1}(x, y)}{\partial x}\right) \dot{x}+\frac{\partial f_{1}(x, y)}{\partial y} \dot{y}, \\
& \dot{v}=\frac{\partial f_{2}(x, y)}{\partial x} \dot{x}+\left(1+\frac{\partial f_{2}(x, y)}{\partial y}\right) \dot{y},
\end{aligned}
$$

Aplicando as expressões de $\dot{u}$ e $\dot{v}$ dadas pelas equações associadas ao campo perturbado (3.2.7), em (3.2.9), , e efetuando-se os cálculos necessários, obtemos:

$$
\left\{\begin{array}{l}
\dot{x}=y+F_{1}(x, y)+\varepsilon G_{1}(x, y), \\
\dot{y}=-\varepsilon x+F_{2}(x, y)+\varepsilon G_{2}(x, y)
\end{array}\right.
$$

onde

$$
\begin{aligned}
& G_{1}(x, y)=\left(x+f_{1}(x, y)\right) \frac{\partial f_{1}(x, y)}{\partial y} \\
& G_{2}(x, y)=\left(-\left(x+f_{1}(x, y)\right) \frac{\partial f_{1}(x, y)}{\partial x}-f_{1}(x, y)\right) .
\end{aligned}
$$

Observe que, $G_{1}$ e $G_{2}$ são funções analíticas, pois $f_{1}$ é uma função analítica.

Seja $X_{\varepsilon}(x, y)$ o campo vetorial associado ao sistema (3.2.10). Uma vez que $Y_{\varepsilon}(u, v)$ tem um centro linear na origem, para todo $\varepsilon>0$, o mesmo vale para $X_{\varepsilon}(x, y)$ e assim, concluímos a demonstração.

De modo simplificado, o teorema acima nos diz que um centro nilpotente analítico é limite de centros lineares analíticos. Do Teorema do Centro Linear, segue que o sistema (3.2.4) admite integral primeira local $H_{\varepsilon}(x, y)$ em $(0,0)$, para todo $\varepsilon>0$. Além disso, se $H(x, y)=\lim _{\varepsilon \rightarrow 0^{+}} H_{\varepsilon}(x, y)$ existe e está bem definido numa vizinhança de $(0,0)$, então $H$ é integral primeira local de (3.2.2).

Logo, o Teorema do Centro Nilpotente reduz o estudo dos centros nilpotentes para o caso dos centros lineares. Podemos aplicar o Algoritmo de Lyapunov - cf. 2.1.3, ao sistema 
(3.2.4), buscando integrais primeiras analíticas da forma $H=\left(\varepsilon x^{2}+y^{2}\right) / 2+F(x, y, \varepsilon)$, onde $F$ começa com termos de ordem maior ou igual a 2 nas variáveis $x$ e $y$. As constantes de Lyapunov $V_{2 k}$ são determinadas a partir de (2.1.21).

Teorema 3.2.5. Suponha que a origem do sistema diferencial analítico real (3.2.2) é monodrômica, e que o sistema é limite de sistemas com centros lineares da forma (3.2.4). Suponha ainda que não existe ponto singular de (3.2.4) tendendo para a origem, quando $\varepsilon$ tende a zero. Então, o sistema (3.2.2) tem um centro na origem.

Demonstração. Considere um sistema analítico da forma (3.2.2), que denotaremos por $(P, Q)$, tendo a origem como ponto singular monodrômico. Suponha que este sistema seja limite de sistemas com centros lineares da forma (3.2.4), denotados por $\left(P_{\varepsilon}, Q_{\varepsilon}\right)$.

Como a origem por hipótese é singularidade monodrômica de $(P, Q)$, se $S$ é uma curva suficientemente pequena com extremidade na origem, então a aplicação de primeiro retorno $\Pi: S \rightarrow S$, associada ao sistema $(P, Q)$ está bem definida e o termo líder de sua expansão de Taylor é linear, basta escolher convenientemente uma curva algébrica semi-transversal, que pode ter uma singularidade no ponto singular - cf. [40].

Por hipótese, a aplicação de primeiro retorno $\prod_{\varepsilon}: S \rightarrow S$ associada ao sistema $\left(P_{\varepsilon}, Q_{\varepsilon}\right)$ é a identidade, $\forall \varepsilon>0$. Logo, pelo Teorema da Dependência Contínua em Relação às Condições Iniciais, seque que $\Pi=\lim _{\varepsilon \rightarrow 0^{+}} \Pi_{\varepsilon}$.

A condição de ausência de pontos singulares tendendo à origem, quando $\varepsilon$ tende a zero, pode ser facilmente verificada usando os termos de menor ordem do sistema perturbado $(3.2 .4)$.

Os Teoremas 3.2.2 e 3.2.5 podem ser usados para detectar centros nilpotentes de sistemas diferenciais analíticos, por meio da aplicação do Algoritmo de Lyapunov - ver Seção 2.1.3. No caso particular de sistemas polinomiais, o método é descrito a seguir. Considere o sistema (3.2.2), com $F_{1}$ e $F_{2}$ polinômios sem termos constantes ou lineares contendo um conjunto de parâmetros arbitrários e tais que a origem é um ponto singular monodrômico. Nestas condições, de acordo com o Teorema 3.2.2, para detectar os centros do sistema precisamos considerar o sistema perturbado

$$
\dot{x}=y+F_{1}(x, y)+\varepsilon G_{1}(x, y), \quad \dot{y}=-\varepsilon x+F_{2}(x, y)+\varepsilon G_{2}(x, y)
$$

onde $G_{1}, G_{2}$ são funções analíticas começando com termos quadráticos em $x$ e $y$. 
Aplicamos então o Algoritmo de Lyapunov para determinar condições necessárias para que a origem do sistema (3.2.12) seja um centro. Em geral, estas condições são satisfeitas escolhendo-se convenientemente os coeficientes das funções analíticas $G_{1}$ e $G_{2}$. Quando isto não é possível, devemos fazer uso dos parâmetros do sistema polinomial não perturbado da forma (3.2.2). Deste modo, obtemos condições necessárias para a existência de um centro da origem deste sistema.

Condições suficientes de centros para um sistema não perturbados da forma (3.2.2) são obtidas em um número finito de passos, pois o Teorema da Base de Hilbert assegura que este processo é finito. A cada vez que encontramos uma condição necessária para que a origem do sistema não perturbado (3.2.2) seja um centro, precisamos conferir se este sistema tem efetivamente um centro na origem. Como o número de passos é finito e para a determinação de cada constante de Lyapunov do sistema perturbado (3.2.12) nós precisamos de apenas um jato finito, a perturbação necessária para detectar os casos de centro são polinômios, isto é, as funções $G_{1}$ e $G_{2}$ são polinômios.

No Capítulo 4, exibimos exemplos de aplicação do Teorema 3.2.2 na investigação de centros em famílias de sistemas diferenciais analíticos. 


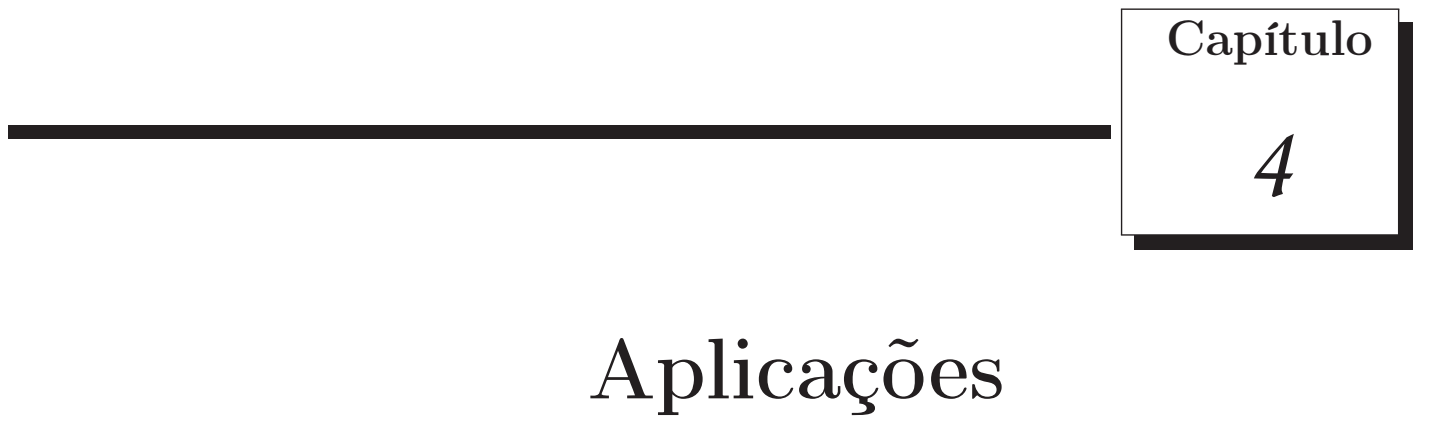

Esta seção é dedicada à aplicação das duas técnicas apresentadas nesta dissertação para investigação do problema do centro-foco em sistemas diferenciais analíticos planares com singularidades nilpotentes.

Primeiramente, utilizamos os Teoremas 3.1.1 e 3.1.10, desenvolvidos por Álvarez \& Gasull - cf. [1, 2], que fazem uso da teoria das formas normais para calcular as constantes de Lyapunov generalizadas, para uma família de sistemas diferenciais planos fixada. Em etapa posterior, aplicamos o Teorema do Centro Nilpotente (Teorema 3.2.2), devido a [29], que investiga centros nilpotentes como limite de centros do tipo linear para uma segunda família.

A aplicação das duas técnicas nos possibilitou avaliar sua eficiência, bem como identificar possíveis dificuldades, tais como o custo computacional envolvido. Vale destacar que a primeira família de sistemas diferenciais analíticos que investigamos é bastante conhecida, tendo sido estudada anteriormente por Gasull \& Torregrosa - cf. [23, 25]. Nossos resultados coincidem parcialmente com os apresentados nestes dois trabalhos, à exceção de uma das condições para centro do sistema. Comentaremos mais a respeito deste assunto ao final deste capítulo.

Para efetuar os cálculos necessários, utilizamos os softwares Maple ${ }^{\circledR}$ v.14.0 e Mathematica ${ }^{\circledR}$ v.8.0, em um PC-Pentium 2.84GHz 4GB RAM. A seguir, apresentamos os resultados obtidos. 


\subsection{Método de Álvarez-Gasull}

Nesta seção, aplicaremos os Teoremas 3.1.1 e 3.1.10, devidos a Álvarez \& Gasull - cf. $[1,2]$, para caracterização do sistema diferencial apresentado a seguir:

$$
\left\{\begin{array}{l}
\dot{x}=-y+p_{3,0} x^{3}+p_{1,1} x y \\
\dot{y}=x^{3}+q_{4,0} x^{4}+q_{2,1} x^{2} y+q_{0,2} y^{2}
\end{array}\right.
$$

onde $p_{i, j}, q_{i, j} \in \mathbb{R}$.

\section{Problema da Monodromia}

Para aplicarmos o Teorema 3.1.1, precisamos que o sistema (4.1.1) seja escrito na forma

$$
\left\{\begin{array}{l}
\dot{x}=y+X_{2}(x, y) \\
\dot{y}=Y_{2}(x, y)
\end{array}\right.
$$

com $X_{2}, Y_{2}$ funções analíticas cujas séries de potências começam, pelo menos, com termos de grau 2. Para isso, basta efetuarmos a mudança de coordenadas $x=-x$ no sistema (4.1.1) obtendo

$$
\left\{\begin{array}{l}
\dot{x}=y-p_{3,0} x^{3}-p_{1,1} x y, \\
\dot{y}=-x^{3}+q_{4,0} x^{4}+q_{2,1} x^{2} y+q_{0,2} y^{2}
\end{array}\right.
$$

e portanto, temos $X_{2}(x, y)=-p_{3,0} x^{3}-p_{1,1} x y$ e $Y_{2}(x, y)=-x^{3}+q_{4,0} x^{4}+q_{2,1} x^{2} y+q_{0,2} y^{2}$. Utilizando a notação do Teorema 3.1.1, e com auxílio do Maple ${ }^{\circledR}$, obtemos as funções $f(x)$ e $\phi(x)$ para o sistema (4.1.1):

$$
\begin{aligned}
f(x) & =Y_{2}(x, F(x))=a x^{\alpha}+O\left(\|x\|^{\alpha+1}\right), a \neq 0, \alpha \geq 2, \\
\stackrel{(4.1 .2)}{\Rightarrow} f(x) & =-x^{3}+q_{4,0} x^{4}+q_{2,1} p_{3,0} x^{5}+\left(q_{2,1} p_{3,0} p_{1,1}+q_{0,2} p_{3,0}{ }^{2}\right) x^{6}+O\left(\|x\|^{7}\right) . \\
\phi(x) & =\left.\operatorname{div}\left(y+X_{2}(x, y), Y_{2}(x, y)\right)\right|_{y=F(x)} \\
& =b x^{\beta}+O\left(\|x\|^{\beta+1}\right), b \neq 0, \beta \geq 1 \text { ou } \phi \equiv 0, \\
\stackrel{(4.1 .2)}{\Rightarrow} \phi(x) & =\left(-3 p_{3,0}+q_{2,1}\right) x^{2}+\left(2 q_{0,2} p_{3,0}-p_{1,1} p_{3,0}\right) x^{3}+O\left(\|x\|^{4}\right) .
\end{aligned}
$$

onde a função $y=F(x)$ é a solução de $y+X_{2}(x, y)=0$ passando por $(0,0)$. 
Consequentemente, temos $a=-1<0, \alpha=3, n=\frac{\alpha+1}{2}=2$. Como a função $\phi$ é da forma $\phi(x)=p_{3,0}\left[-3 x^{2}+\frac{p_{1,1} x^{3}}{-1+p 1,1 x}-\frac{2 q_{0,2} x^{3}}{-1+p_{1,1} x}\right]+q_{2,1} x^{2}$, segue que $\phi(x) \equiv 0 \Leftrightarrow$ $p_{3,0}=q_{2,1}=0$. Esta relação, de fato, será a primeira condição de centro para o sistema (4.1.2), como veremos mais tarde.

Se $\phi(x) \not \equiv 0$, então $\beta=2$. Então, $2=\beta>n-1=1$, e consequentemente, o sistema (4.1.2) satisfaz a condição $(i)$ do Teorema 3.1.1.

Portanto, o sistema (4.1.1) satisfaz as condições do Teorema 3.1.1, e desta forma, possui uma singularidade nilpotente monodrômica na origem. A seguir, apresentamos os detalhes.

\section{Problema da Estabilidade}

Para aplicarmos o Teorema 3.1.10 e caracterizarmos os centros deste sistema, é necessário realizar alguns passos preliminares.

\section{Forma Normal}

Utilizando o Teorema da Forma Normal, obtivemos, com o auxílio do Maple ${ }^{\circledR}$, a forma normal do sistema (4.1.1), com truncamento de ordem 7:

$$
\left\{\begin{array}{l}
\dot{x}=-y \\
\dot{y}=a(x)+y \tilde{b}(x)
\end{array}\right.
$$

onde:

$$
\begin{aligned}
a(x)= & x^{3}+\left(-\frac{1}{2} q_{0,2}-\frac{3}{2} p_{1,1}+q_{4,0}\right) x^{4}+\left(q_{2,1} p_{3,0}+\frac{3}{2} p_{1,1} q_{0,2}+\frac{5}{4} p_{1,1}{ }^{2}+\frac{1}{4} q_{0,2}^{2}\right. \\
& \left.-q_{4,0} q_{0,2}-2 q_{4,0} p_{1,1}\right) x^{5}+\left(-\frac{5}{4} p_{1,1} q_{0,2}{ }^{2}-\frac{15}{8} p_{1,1}^{2} q_{0,2}-\frac{1}{8} q_{0,2}{ }^{3}+\frac{5}{6} q_{4,0} q_{0,2}{ }^{2}\right. \\
& \left.+3 q_{4,0} p_{1,1} q_{0,2}-\frac{3}{4} p_{1,1}^{3}-\frac{3}{2} q_{2,1} p_{3,0} p_{1,1}-\frac{3}{2} q_{2,1} p_{3,0} q_{0,2}+\frac{13}{6} q_{4,0} p_{1,1}{ }^{2}+q_{0,2} p_{3,0}{ }^{2}\right) x^{6} \\
& +\left(p_{1,1} q_{0,2}{ }^{3}+\frac{25}{12} p_{1,1}^{2} q_{0,2}^{2}+3 q_{2,1} p_{3,0} p_{1,1} q_{0,2}+\frac{7}{120} q_{0,2}^{4}+\frac{3}{2} p_{1,1}{ }^{3} q_{0,2}-\frac{2}{3} q_{4,0} q_{0,2}{ }^{3}\right. \\
& -\frac{5}{3} q_{4,0} p_{1,1}^{3}-\frac{13}{3} q_{4,0} p_{1,1}^{2} q_{0,2}+\frac{5}{3} q_{2,1} p_{3,0} q_{0,2}^{2}-\frac{10}{3} q_{4,0} p_{1,1} q_{0,2}{ }^{2}+\frac{43}{120} p_{1,1}^{4} \\
& \left.+\frac{4}{3} q_{2,1} p_{3,0} p_{1,1}^{2}-2 q_{0,2}^{2} p_{3,0}^{2}-q_{0,2} p_{3,0}^{2} p_{1,1}\right) x^{7}
\end{aligned}
$$




$$
\begin{aligned}
& \tilde{b}(x)=\left(3 p_{3,0}+q_{2,1}\right) x^{2}+\left(-q_{0,2} p_{3,0}-q_{2,1} q_{0,2}-2 p_{1,1} p_{3,0}-q_{2,1} p_{1,1}\right) x^{3}+\left(2 p_{1,1} p_{3,0} q_{0,2}\right. \\
& \left.+\frac{5}{4} p_{1,1}^{2} p_{3,0}-\frac{1}{4} q_{0,2}^{2} p_{3,0}+\frac{3}{2} q_{2,1} p_{1,1} q_{0,2}+\frac{7}{12} q_{2,1} p_{1,1}^{2}+\frac{11}{12} q_{2,1} q_{0,2}^{2}\right) x^{4} \\
& +\left(-\frac{1}{2} p_{3,0} p_{1,1}{ }^{3}-\frac{5}{6} q_{2,1} q_{0,2}{ }^{3}-\frac{1}{4} q_{2,1} p_{1,1}^{3}-2 p_{3,0} p_{1,1}^{2} q_{0,2}-\frac{3}{2} p_{3,0} p_{1,1} q_{0,2}^{2}\right. \\
& \left.+q_{0,2}{ }^{3} p_{3,0}-\frac{7}{4} q_{2,1} q_{0,2}{ }^{2} p_{1,1}-\frac{7}{6} q_{2,1} q_{0,2} p_{1,1}^{2}\right) x^{5}+\left(-\frac{22}{15} q_{0,2}{ }^{4} p_{3,0}+\frac{137}{180} q_{2,1} q_{0,2}{ }^{4}\right. \\
& +\frac{31}{360} q_{2,1} p_{1,1}^{4}+\frac{7}{40} p_{1,1}^{4} p_{3,0}+\frac{11}{12} q_{0,2}^{3} p_{3,0} p_{1,1}+\frac{55}{24} q_{0,2}^{2} p_{3,0} p_{1,1}^{2}+\frac{13}{12} p_{1,1}^{3} p_{3,0} q_{0,2} \\
& \left.+\frac{15}{8} q_{2,1} q_{0,2}^{3} p_{1,1}+\frac{119}{72} q_{2,1} q_{0,2}{ }^{2}{p_{1,1}}^{2}+5 / 8 q_{2,1} p_{1,1}{ }^{3} q_{0,2}\right) x^{6}+\left(-\frac{7}{10} q_{2,1} q_{0,2}{ }^{5}\right. \\
& -\frac{1}{40} q_{2,1} p_{1,1}{ }^{5}-\frac{1}{20} p_{1,1}{ }^{5} p_{3,0}+\frac{53}{30} q_{0,2}{ }^{5} p_{3,0}-\frac{25}{24} q_{2,1} q_{0,2}{ }^{2} p_{1,1}{ }^{3}-\frac{29}{15} q_{2,1} q_{0,2}{ }^{4} p_{1,1} \\
& -\frac{31}{120} q_{2,1} q_{0,2} p_{1,1}^{4}-\frac{49}{24} q_{2,1} q_{0,2}{ }^{3}{p_{1,1}}^{2}-\frac{55}{24} q_{0,2}{ }^{3} p_{3,0} p_{1,1}{ }^{2}-\frac{19}{12} q_{0,2}^{2} p_{3,0} p_{1,1}{ }^{3} \\
& \left.-\frac{11}{30} q_{0,2}^{4} p_{3,0} p_{1,1}-\frac{19}{40} q_{0,2} p_{3,0} p_{1,1}^{4}\right) x^{7}
\end{aligned}
$$

\section{Aplicação do Lema 3.1.8}

De (4.1.3), segue que $a(x)=x^{3}(1+O(\|x\|))$. Logo, $2 n-1=3$, isto é, $n=2$. Podemos então escrever a função $\Phi^{-1}(u)$, do Lema 3.1.8, para o sistema (4.1.3): $\Phi(x)=$ $x \sqrt[4]{1+O(\|x\|)}$.

Utilizando o Maple ${ }^{\circledR}$ para:

- expandir a função $\Phi^{-1}(u)$ em série de Taylor até ordem 7,e

- calcular a forma normal de $\Phi^{-1}(u)$ até o grau 7 ,

obtemos o sistema:

$$
\left\{\begin{array}{l}
\dot{x}=-y, \\
\dot{y}=x^{2 n-1}+y b(x),
\end{array}\right.
$$

onde $b(x)$ é dado por:

$$
\begin{aligned}
& b(x)=\sum_{j \geq \beta} b_{j} x^{j}, b_{\beta} \neq 0 \\
& =A x^{2}+B x^{3}+C x^{4}+D x^{5}+E x^{6} .
\end{aligned}
$$


onde $A, B, C, D, E$ são funções de $p_{i, j}, q_{i, j}$, dadas no Anexo A.

\section{Teorema 3.1.10}

Com os resultados obtidos, finalmente podemos aplicar o Teorema 3.1.10.

Da expressão de $b(x)$, obtida anteriormente, segue que $\beta=2 l=2$ e portanto, $2=$ $\beta>n-1=1$. Desta forma, estamos na condição 1.(a) do Teorema 3.1.10.

Segue que $2-n+2 l=2$ e desta forma, a primeira constante de Lyapunov generalizada significativa é $V_{2}$. Efetuando-se os cálculos necessários, com o auxílio do Maple ${ }^{\circledR}$, obtemos $V_{2}=K_{2}\left(3 p_{3,0}+q_{2,1}\right)$.

Procedendo de forma análoga, obtemos $V_{4}=K_{4}\left(-\frac{1}{3} q_{2,1}\left(2 q_{0,2}+p_{1,1}\right)\left(p_{1,1}-q_{0,2}-q_{4,0}\right)\right) \mathrm{e}$ $V_{6}=K_{6}\left(\frac{1}{216} q_{2,1}\left(2 q_{0,2}+p_{1,1}\right)\left(4 q_{0,2}+9 q_{4,0}\right)\left(3 q_{4,0} q_{0,2}+6 q_{4,0}^{2}+2 q_{2,1}^{2}\right)\right)$. Se a origem é um centro, todas as constantes generalizadas são nulas. Assim, obtemos as seguintes condições:

(a) $p_{3,0}=q_{2,1}=0$;

(b) $p_{1,1}+2 q_{0,2}=q_{2,1}+3 p_{3,0}=0$;

(c) $4 p_{1,1}+5 q_{4,0}=q_{2,1}+3 p_{3,0}=4 q_{0,2}+9 q_{4,0}=0$;

(d) $p_{1,1}-q_{0,2}-q_{4,0}=6 p_{3,0}^{2}+q_{0,2} q_{4,0}+2 q_{4,0}^{2}=q_{2,1}+3 p_{3,0}=0$.

\section{Discussão dos resultados}

A técnica de Alvarez \& Gasull - cf. [1, 2] faz uso da teoria das formas normais para calcular as constantes de Lyapunov generalizadas. A aplicação desta técnica numa família concreta de sistemas diferenciais nos permitiu identificar alguns aspectos importantes, que destacamos a seguir.

- Truncamento da forma normal: a aplicação da técnica exige truncamento da forma normal até ordem conveniente. Entretanto, o método não explicita qual deve ser esta ordem. Outros truncamentos necessários, como a ordem da expansão da série de Taylor da função inversa $\Phi^{-1}$ (vide Lema 3.1.8), também requerem cuidado, uma vez que truncar a série em ordem muito baixa pode resultar em expressões que não contemplam todas as condições de centro do sistema analisado; 
- Custo computacional: a aplicação da técnica mostrou-se computacionalmente dispendiosa. A obtenção da forma normal e da função inversa $\Phi^{-1}$, bem como os demais cálculos necessários à aplicação do Teorema 3.1.10, demandaram considerável tempo de execução do computador utilizado para a pesquisa - PC Pentium $2.84 \mathrm{GHz}$ 4GB de RAM. Para os cálculos com formas normais truncadas em grau maior que 9, e com expansão de Taylor de $\Phi^{-1}$ superior a 8 , foi preciso recorrer a máquina com mais recursos, nesse caso, um MacAir i7 1.8GHz dual chip 8GB de RAM;

- Constantes de Lyapunov generalizadas: a afirmação (2) do Teorema 3.1.10 fornece condições necessárias e suficientes para que a origem seja um centro. Na prática, no entanto, calculamos as constantes de Lyapunov generalizadas e verificamos as condições para que sejam iguais a zero, uma vez que a origem é um centro se, e somente se, todas as constantes forem nulas. Entretanto, o método também não fornece a quantidade de constantes generalizadas que precisam ser calculadas para obter todas as condições de centro de um dado sistema.

Com relação aos resultados obtidos para a família de sistemas diferenciáveis analíticos (4.1.1), destacamos que nossos resultados diferem dos apresentados por Gasull \& Torregrosa - cf. [23, 25], em uma das condições, a saber, a condição (c). Em seu lugar, os referidos autores apresentam a condição $2 p_{1,1}+q_{4,0}=q_{2,1}+3 p_{3,0}=4 q_{0,2}+9 q_{4,0}=0$. Não identificamos erros em nossos cálculos e tampouco fomos capazes de mostrar que a condição obtida em nossos trabalhos ou aquela apresentada por Gasull \& Torregrosa não é válida. Vale observar que, devido a dificuldades computacionais, a investigação da mesma família de sistemas diferenciais segundo a abordagem apresentada na próxima seção, não pôde ser concluída.

\subsection{Método de Giacomini-Giné-Llibre}

Nesta seção, discutimos a aplicação do Teorema do Centro Nilpotente (Teorema 3.2.2) - cf. [29], que investiga centros nilpotentes como limite de centros do tipo linear. Para tanto, vamos aplicar o método para uma família fixada de sistemas diferenciais proposta em [29]. Vale ressaltar que os resultados obtidos em [29] coincidem parcialmente com os apresentados abaixo, devido a uma diferença nas expressões de $G_{1}$ e $G_{2}$, corrigida posteriormente pelos autores do paper. Para efetuar os cálculos, utilizamos os softwares Mathematica ${ }^{\circledR}$ e Maple ${ }^{\circledR}$. 
Considere a seguinte família de sistemas:

$$
\left\{\begin{array}{l}
\dot{x}=-y+x^{2}+k_{2} x y \\
\dot{y}=k_{1} x^{2}-x^{3}, \quad k_{1}, k_{2} \in \mathbb{R}
\end{array}\right.
$$

Vamos aplicar o Algoritmo de Lyapunov - cf. 2.1.3 ao sistema perturbado abaixo:

$$
\left\{\begin{array}{l}
\dot{x}=-y+x^{2}+k_{2} x y+\varepsilon G_{1}(x, y) \\
\dot{y}=k_{1} x^{2}-x^{3}+\varepsilon G_{2}(x, y)
\end{array}\right.
$$

onde $\varepsilon>0$, e $G_{1}(x, y)=\sum_{i+j \geq 2}^{7} a_{i j} x^{i} y^{j}, G_{2}(x, y)=\sum_{i+j \geq 2}^{7} b_{i j} x^{i} y^{j}$. Vale ressaltar que, nas expansões de $G_{1}$ e $G_{2}$ não encontramos monômio da forma $\alpha y^{2}, \alpha \in \mathbb{R}$, devido à forma como foram definidas em (3.2.11). Utilizando o software Mathematica ${ }^{\circledR}$, obtivemos a primeira constante de Lyapunov:

$$
\begin{aligned}
V_{1}= & \frac{1}{8 \varepsilon^{\frac{3}{2}}}\left[2 k_{1}+\left(2 b_{20}+2 a_{20} k_{1}+b_{11} k_{1}-k_{2}\right) \varepsilon\right. \\
& \left.+\left(-a_{11}+3 a_{30}+2 a_{20} b_{20}+b_{11} b_{20}+b_{21}-a_{20} k_{2}\right) \varepsilon^{2}+\left(a_{12}-a_{11} a_{20}+3 b_{03}\right) \varepsilon^{3}\right] .
\end{aligned}
$$

Observe que $V_{1}$ possui apenas coeficientes dos termos quadráticos e cúbicos das funções $G_{1}$ e $G_{2}$. Para que $V_{1}$ se anule, precisamos que todos os coeficientes de cada potência de $\varepsilon$ seja zero. Assim, obtemos a condição necessária para centro $k_{1}=0$ para o sistema (4.2.1) e para uma perturbação arbitrária. Também obtemos as condições $b_{20}=k_{2} / 2$, $b_{21}=a_{11}-3 a_{30}-b_{11} b_{20}$ e $b_{03}=\left(-a_{12}+a_{11} a_{20}\right) / 3$.

A segunda constante de Lyapunov é:

$$
V_{2}=\frac{1}{192 \varepsilon^{\frac{7}{2}}}\left[-36 k_{2} \varepsilon^{2}+O\left(\|\varepsilon\|^{3}\right)\right]
$$

$\mathrm{Na}$ expressão de $V_{2}$ temos contribuições dos coeficientes dos termos de grau 2 até grau 5 de $G_{1}$ e $G_{2}$.

Assim, as condições $k_{1}=k_{2}=0$ são necessárias para que a origem do sistema (4.2.1) seja um centro. Estas condições também são suficientes, como mostraremos a seguir. 
Proposição 4.2.1. O sistema

$$
\left\{\begin{array}{l}
\dot{x}=-y+x^{2}, \\
\dot{y}=-x^{3},
\end{array}\right.
$$

tem um centro nilpotente na origem.

Demonstração. Primeiramente, vamos aplicar o Teorema 3.1.1 para verificar que a origem do sistema (4.2.2) é monodrômica. As funções $f(x)$ e $\phi(x)$, conforme Teorema 3.1.1 neste caso são bastante simples:

$$
\left\{\begin{array}{l}
f(x)=-x^{3}, \\
\phi(x)=2 x
\end{array}\right.
$$

Seque que $a=-1<0, \alpha=3, n=2, b=2$ e $\beta=1$. Assim, $\beta=1=n-1$ e $b^{2}+4 a n=-4<0$ e pela condição (ii) do Teorema 3.1.1, a origem do sistema (4.2.2) é monodrômica.

O próximo passo é mostrar que a origem do sistema (4.2.2) é de fato um centro. Com o auxílio do Maple ${ }^{\circledR}$, escrevemos o sistema (4.2.2) na forma:

$$
\left\{\begin{array}{l}
\dot{x}=-y, \\
\dot{y}=x^{3}+y(-2 x) .
\end{array}\right.
$$

Logo, $b(x)=-2 x$, isto é, $b^{e}(x) \equiv 0$ e pelo item 2 do Teorema 3.1.10, a origem é um centro.

Vale salientar que, apesar da origem do sistema (4.2.2) ser um centro, este sistema não possui uma primeira integral analítica local, e nem uma primeira integral formal definida na origem - cf. [15]. Esse Exemplo portanto, ilustra a impossibilidade de estendermos o Teorema do Centro Linear - cf. Teorema 3.2.1, para sistemas com singularidades degeneradas.

O procedimento apresentado no exercício anterior para investigação de centros nilpotentes é usual na literatura. Ou seja, uma vez obtidas condições necessárias para a singularidade ser um centro, utilizamos outros resultados e técncas que nos permitam obter condições condições suficientes. Tais resultados, em geral, são específicos para a família 
a ser investigada. Este fato é uma das grandes dificuldades na caracterização de centros nilpotentes. 


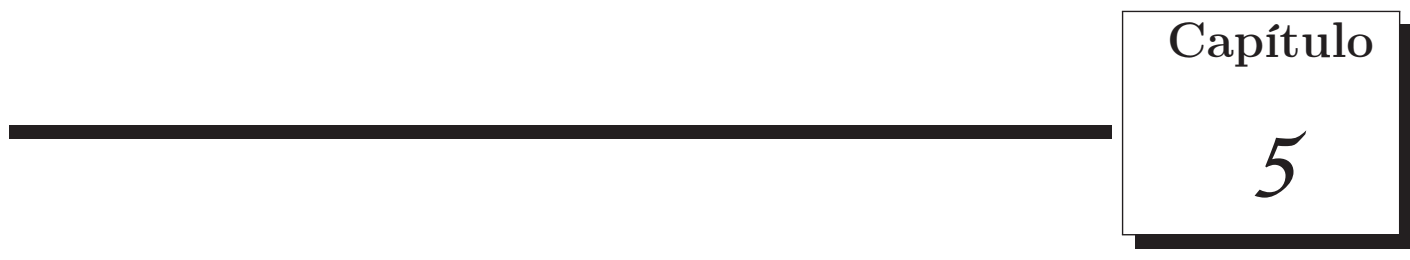

\section{Conclusões}

O estudo de duas técnicas distintas para investigação do problema do centro-foco em sistemas analíticos com singularidade nilpotente nos permitiu conhecer, em profundidade, este que é um dos problemas clássicos da teoria qualitativa das EDO's. A aplicação das técnicas em famílias concretas de sistemas diferenciais possibilitou verificar as dificuldades computacionais e demais obstruções, bem como avaliar a eficiência de cada um dos métodos.

Pretendemos dar continuidade ao estudo de sistemas diferenciais analíticos planares com centros do tipo nilpotente e degenerado com parte linear identicamente nula, em particular, sistemas diferenciais polinomiais de grau 3 e 4 . Conforme observado em [26], o problema do centro-foco está fortemente relacionado ao $16^{\circ}$ Problema de Hilbert e à caracterização de sistemas diferenciais com integral primeira. Deste modo, também pretendemos investigar questões relacionadas à bifurcação de ciclos limites e a sistemas diferenciais com integral primeira local. 


\section{Referências Bibliográficas}

[1] M. J. Álvarez; A. Gasull. Monodromy and Stability of Nipotent Critical Points, I. J. Bifurcation and Chaos (2005) 1253-1265.

[2] M. J. Álvarez; A. Gasull. Generating limit cycles form a nilpotent critical point via normal forms, J. Math. Anal. Appl. 318 (2006) 271-287.

[3] $\Lambda$ Andreev. Investigation of the behaviour of the integral curves of a system of two differential equations in the neighborhood of a singular point, Transl. Amer. Math. Soc. 8 (1958) 187-207.

[4] A. A. Andronov; E. A. Leonvich; I. I. Gordon; and A. G. Maier. Qualitative yheory of second-order dynamic systems, John Wiley \& Sons, NY, 1973.

[5] A. A. Andronov; E. A. Leonvich; I. I. Gordon; and A. G. Maier. Theory of bifurcation of dynamical systems on a plane, John Wiley \& Sons , NY, 1973.

[6] V. I. Arnol'd. Index of a singular point of a vector field, the Petrovskii-Oleinik inequality, and mixed hodge structures, Funct. Anal. Appl., 12 (1978) 1-12.

[7] V. I. Arnol'd; Y. S. Ilyashenko. Ordinary Differential Equations, Encyclopedia Math. Sci. 1, Springer, Berlim, 1988.

[8] D. K. Arrowsmith; C. M. Place. An Introduction to Dynamical Systems, Cambridge University Press, 1990.

[9] E. Batcheva; N. B. Medvedeva. The second terms of the assymtoptics of the monodromy map in case of two even edges of newton diagram, Electron. J. Qualit. Th. Diff. Eqs. 19 (2000) 1-15. 
[10] N. N. Bautin. On the number of limit cycles which appear with the variation of coefficients from an equilibrium position of focus or center type., Amer. Math. Soc. Translation 100 (1962) 1-19.

[11] M. Berthier; R. Moussu. Réversibilité et classification des centres nilpotents, Am. Inst. Fourier (Grenoble) 44 (1994) 465-494.

[12] L. C. V. Estudo do teorema de Bautin para campos quadráticos no plano. 2011. 88 p. Dissertação (Mestrado). Programa de Pós-Graduação em Física e Matemática Aplicada, Universidade Federal de Itajubá, Itajubá, 2011.

[13] M. Brunella. Topological equivalence of a plane vector field with its principal part defined through Newton polyhedra, J. Differential Equations 85 (1990) 338-366.

[14] J. Chavarriga; H. Giacomini; J. Giné; J. Llibre. On the integrability of twodimensional flows, J. Diffrential Equations 157 (1999) 163-182.

[15] J. Chavarriga; H. Giacomini; J. Giné; J. Libbre. Local analytic integrability for nilpotent centers, Ergodic Theory Dynam. Systems 23 (2003) 417-428.

[16] C. Chicone.Ordinary Differential Equations with Applications, Applied Mathematics, Springer-Verlag, New York, 1999.

[17] S. N. Chow; C. Li; D. Wang. Normal forms and bifurcation of planar vector fields, Cambridge University Press, 1994.

[18] C. Christopher. Advanced course on Limit cycles of differential equations. Notes of the course, Centre de Recerca Matemàtica, Bellaterra, Espanha, 2006.

[19] A. Cima; A. Gasull; F. Mañosas. Ciclycity of a Family of Vector Fields, J. Math. Anal. Appl. 196 (1995) 921-937.

[20] T. V. Davies; E. M. James. Nonlinear Differential Equations, Addison-Wesley Publishing Company, 1966.

[21] M. Frommer. Über sas aufreten von wirblen und strudeln (geschlossener und spiraliger integralkurven) in der ungebung rationaler unbestimmheitssltellen, , Math. Ann. 109 (1934), 395-424.

[22] F. Dumortier; J. Llibre; and J. C. Artés. Qualitative theory of planar differential systems, UniversiText, Springer-Verlag, 2006. 
[23] A. Gasull, J. Torregrosa. Centre problem for several differential equations via Cherka's method, J. Math. Anal. Appl. 228 (2) (1998) 322-343.

[24] A. Gasull; J. Llibre; Victor Mañosa; Francesc Mañosas. The Focus-Centre Problem for a Type of Degenerate System, Nonlinearity 13 (2000) 699-729.

[25] A. Gasull; J. Torregrosa. A new algorithm for the computation of the Lyapunov constants for some degenerate critical points, Nonlin. Anal. 47 (2001) 4479-4490.

[26] A. Gasull; J. Giné. Cyclicity versus center problem, Qual. Theory Dyn. Syst. 9 (2010), no. 1-2 101-113.

[27] J. Giné. Sufficient conditions for a center at a completely degenerate critical point, Internat. J. Bifur. Chaos Appl. Sci. Engrg. 12 (2002), no. 7, 1659-1666.

[28] J. Giné. On some open problems in planar differential systems and Hilbert's 16th problem, Chaos, Solitons and Fractals 31 (2007) 1118-1134.

[29] H. Giacomini; J. Giné; J. Llibre. The problem of distinguishing between a center and a focus for nilpotent and degenerate analytic systems, J. Differential Equations $\mathbf{2 2 7}$ (2006) 406-426.

[30] J. Guckenheimer; P. Holmes. Nonlinear oscilattions, dynamical systems, and bifurcations of vector fields, Applied Mathematical Sciences 42, Springer, 1983.

[31] M. Hirsch; S. Smale. Differential Equation, Dynamical Systems and Linear Algebra, Academic Press, 1974.

[32] Y. S. Ilyashenko. Algebraic nonsolvability and almost algebraic solvability of the center-focus problem, Funct. Anal. Appl. 6 (1972) 30-37.

[33] Y. S. Ilyashenko. Finiteness theorems for limit cycles, Translations of Math. Monographs, 94, Amer. Math. Soc., Providence, R.I., 1991.

[34] N. V. Kuznetsov. Computation of Lyapunov quantities, $6^{\text {th }}$ EUROMECH Nonlinear Dynamics Conference, 2008.

[35] W. Li; J. Llibre; M. Nicolau, X. Zhang. On the differentiability of the first integrals of two-dimensional flows, Proc. Amer. Math. Soc. 130 (2002) 2079-2088. 
[36] M. A. Lyapunov. Problème général de la stabilité du mouvement, Ann. of Math. Stud. 17, Princeton University Press, 1947.

[37] M. A. Lyapunov Stability of Motion, Mathematics in Science and Engineering, 30, Academic Press, NY-London, 1966.

[38] V. Mañosa. On the center problem for degenerate singular points of planar vector fields, Int. J. Bifurcation and Chaos, 12 (2002) 687-707.

[39] L. Mazzi; M. Sabatini. A characterization of centres via first integrals, J. Differential Equations 76 (1988) 222-237.

[40] M. D. Medvedeva. The principal term of the monodromy transformation of a monodromic singular point is linear, Siberian Math. J. 33 (1992) 280-288.

[41] R. Moussu. Symétrie et forme normale des centres et foyers dégénérés, Ergod. Th. Dyn. Syst. 2 (1982) 241-251.

[42] R. Moussu. Une démonstration d'un théorème de Lyapunov-Poincaré, Astérisque 98/99 (1982) 216-223.

[43] J. Palis Jr; W. Melo. Introdução aos Sistemas Dinâmicos, IMPA/ CNPq, 1977.

[44] L. Perko. Differential Equations and Dynamical Systems, 2nd. ed., Springer-Verlag, 1996.

[45] H. Poincaré. Mémoire sur les courbes définies par les équations différentielles, Journal de Mathématiques 37 (1881) 375-422; 8 (1882) 251-296; Oeuvres de Henri Poincaré, vol. I, Gauthier-Villars, Paris, 1951, pp. 3-84.

[46] L. S. Pontryagin. Ordinary Differential Equations, Addison-Wesley Publishing Company, 1962.

[47] V. G. Romanovski; Shafer, D. S. The center and cyclicity problems: a computational algebra approach, Boston-Basel-Berlin: Birkhauser, 2009.

[48] J. Sotomayor. Lições de equações diferenciais, Projeto Euclides, CNPq, 1979.

[49] J. Sotomayor. Curvas definidas por equações diferenciais no plano, $13^{\circ}$. Colóquio Brasileiro de Matemática, 1981. 
[50] E. Stróżyna; H. Żoładek. The analytic and formal normal form for the nilpotent singularity. J. Differential Equations 179 (2002) 479-537.

[51] F. Takens. Singularities of vector fields, Inst. Hautes Études Sci. Publ. Math. 43 (1974) 47-100.

[52] S. Wiggins. Introduction to applied nonlinear dynamical systems and chaos, Texts in Applied Mathematics 2, Springer, 1990.

[53] Ye Yan-Qian at al. Theory of limit cycles, Translations of Mathematical Monographs 66 Amer. Math. Soc. Translation, Providence, RI 1986.

[54] C. Zuppa. Order of Cyclicity of the Singular Point of Liénard's Polynomial Vector Fields, Bol. Soc. Brasil Math. 12 (1981) 105-111. 


\section{Apêndice}

\section{Componentes da função $b(x)$}

$$
\begin{aligned}
A & =\left(3 p_{3,0}+q_{2,1}\right) \\
B & =\left(3 p_{3,0}+q_{2,1}\right)\left(\frac{1}{4} q_{0,2}+\frac{3}{4} p_{1,1}-\frac{1}{2} q_{4,0}\right)-q_{0,2} p_{3,0}-2 p_{1,1} p_{3,0}-q_{2,1} q_{0,2}-q_{2,1} p_{1,1} \\
& +\left(-3 p_{3,0}-q_{2,1}\right)\left(-\frac{1}{8} q_{0,2}-\frac{3}{8} p_{1,1}+\frac{1}{4} q_{4,0}\right) \\
C & =\left(( - 3 p _ { 3 , 0 } - q _ { 2 , 1 } ) \left(\frac{87}{64} p_{1,1} q_{0,2}+\frac{229}{128} p_{1,1}^{2}-\frac{79}{32} q_{4,0} p_{1,1}+\frac{29}{128} q_{0,2}^{2}-\frac{29}{32} q_{4,0} q_{0,2}+\frac{21}{32} q_{4,0}^{2}\right.\right. \\
& +\frac{1}{4} q_{2,1} p_{3,0}+\left(\frac{1}{8} q_{0,2}+\frac{3}{8} p_{1,1}-\frac{1}{4} q_{4,0}\right)^{2}+\left(\frac{1}{8} q_{0,2}+\frac{3}{8} p_{1,1}-\frac{1}{4} q_{4,0}\right)\left(\frac{1}{4} q_{0,2}\right. \\
& \left.\left.+\frac{3}{4} p_{1,1}-\frac{1}{2} q_{4,0}\right)+\left(-\frac{1}{2} q_{0,2}-\frac{3}{2} p_{1,1}+q_{4,0}\right)\left(\frac{1}{2} q_{0,2}+\frac{3}{2} p_{1,1}-q_{4,0}\right)\right)+\left(3 p_{3,0}+q_{2,1}\right) \\
& \left(-\frac{3}{32} p_{1,1} q_{0,2}+\frac{23}{64} p_{1,1}^{2}-\frac{5}{16} q_{4,0} p_{1,1}-\frac{1}{64} q_{0,2}^{2}+\frac{1}{16} q_{4,0} q_{0,2}+\frac{7}{16} q_{4,0}{ }^{2}-\frac{1}{2} q_{2,1} p_{3,0}\right. \\
& \left.+\left(\frac{1}{8} q_{0,2}+\frac{3}{8} p_{1,1}-\frac{1}{4} q_{4,0}\right)^{2}\right)+\left(-q_{0,2} p_{3,0}-2 p_{1,1} p_{3,0}-q_{2,1} q_{0,2}-q_{2,1} p_{1,1}\right)\left(\frac{3}{8} q_{0,2}+\frac{9}{8} p_{1,1}\right. \\
& \left.-\frac{3}{4} q_{4,0}\right)+\frac{11}{12} q_{2,1} q_{0,2}^{2}+\frac{3}{2} q_{2,1} q_{0,2} p_{1,1}+\frac{7}{12} q_{2,1} p_{1,1}{ }^{2}-\frac{1}{4} q_{0,2}^{2} p_{3,0}+2 p_{1,1} p_{3,0} q_{0,2} \\
& +\frac{5}{4} p_{1,1}^{2} p_{3,0}+\left(-\frac{1}{8} q_{0,2} p_{3,0}-\frac{11}{8} p_{1,1} p_{3,0}+\frac{9}{4} p_{3,0} q_{4,0}+\frac{5}{8} q_{2,1} q_{0,2}-\frac{1}{8} q_{2,1} p_{1,1}\right. \\
& \left.\left.+\frac{3}{4} q_{2,1} q_{4,0}\right)\left(-\frac{1}{8} q_{0,2}-\frac{3}{8} p_{1,1}+\frac{1}{4} q_{4,0}\right)\right)
\end{aligned}
$$




$$
\begin{aligned}
& D=\left(-3 p_{3,0}-q_{2,1}\right)\left(( - 1 / 2 q _ { 0 , 2 } - 3 / 2 p _ { 1 , 1 } + q _ { 4 , 0 } ) \left(-3 / 16 p_{1,1} q_{0,2}+\frac{23}{32} p_{1,1}{ }^{2}-5 / 8 q_{4,0} p_{1,1}\right.\right. \\
& -1 / 32 q_{0,2}^{2}+1 / 8 q_{4,0} q_{0,2}+\frac{7}{8} q_{4,0}^{2}-q_{2,1} p_{3,0}+2\left(1 / 8 q_{0,2}+3 / 8 p_{1,1}-1 / 4 q_{4,0}\right)^{2}+\left(1 / 4 q_{0,2}\right. \\
& \left.\left.+3 / 4 p_{1,1}-1 / 2 q_{4,0}\right)^{2}\right)+\left(1 / 8 q_{0,2}+3 / 8 p_{1,1}-1 / 4 q_{4,0}\right)\left(-\frac{3}{32} p_{1,1} q_{0,2}+\frac{23}{64} p_{1,1}{ }^{2}\right. \\
& -\frac{5}{16} q_{4,0} p_{1,1}-\frac{1}{64} q_{0,2}^{2}+1 / 16 q_{4,0} q_{0,2}+\frac{7}{16} q_{4,0}^{2}-1 / 2 q_{2,1} p_{3,0}+\left(1 / 8 q_{0,2}+3 / 8 p_{1,1}\right. \\
& \left.\left.-1 / 4 q_{4,0}\right)^{2}\right)+3 a_{4}+2\left(1 / 8 q_{0,2}+3 / 8 p_{1,1}-1 / 4 q_{4,0}\right)\left(-\frac{3}{64} p_{1,1} q_{0,2}+\frac{23}{128} p_{1,1}{ }^{2}\right. \\
& \left.-\frac{5}{32} q_{4,0} p_{1,1}-\frac{1}{128} q_{0,2}^{2}+1 / 32 q_{4,0} q_{0,2}+\frac{7}{32} q_{4,0}^{2}-1 / 4 q_{2,1} p_{3,0}\right)+\left(-\frac{3}{64} p_{1,1} q_{0,2}\right. \\
& \left.+\frac{23}{128} p_{1,1}^{2}-\frac{5}{32} q_{4,0} p_{1,1}-\frac{1}{128} q_{0,2}^{2}+1 / 32 q_{4,0} q_{0,2}+\frac{7}{32} q_{4,0}^{2}-1 / 4 q_{2,1} p_{3,0}\right) \\
& \left(1 / 4 q_{0,2}+3 / 4 p_{1,1}-1 / 2 q_{4,0}\right)+\left(1 / 4 q_{0,2}^{2}-q_{4,0} q_{0,2}+5 / 4 p_{1,1}^{2}+3 / 2 p_{1,1} q_{0,2}-2 q_{4,0} p_{1,1}\right. \\
& \left.+q_{2,1} p_{3,0}\right)\left(5 / 8 q_{0,2}+\frac{15}{8} p_{1,1}-5 / 4 q_{4,0}\right)-5 / 4 p_{1,1} q_{0,2}{ }^{2}-\frac{15}{8} p_{1,1}^{2} q_{0,2}-1 / 8 q_{0,2}^{3} \\
& -3 / 4 p_{1,1}^{3}+q_{0,2} p_{3,0}^{2}+5 / 6 q_{4,0} q_{0,2}^{2}+3 q_{4,0} p_{1,1} q_{0,2}+\frac{13}{6} q_{4,0} p_{1,1}^{2}-3 / 2 q_{2,1} p_{3,0} p_{1,1} \\
& \left.-3 / 2 q_{2,1} p_{3,0} q_{0,2}\right)+\left(-q_{0,2} p_{3,0}-2 p_{1,1} p_{3,0}-q_{2,1} q_{0,2}-q_{2,1} p_{1,1}\right)\left(-\frac{9}{64} p_{1,1} q_{0,2}\right. \\
& +\frac{69}{128} p_{1,1}^{2}-\frac{15}{32} q_{4,0} p_{1,1}-\frac{3}{128} q_{0,2}^{2}+\frac{3}{32} q_{4,0} q_{0,2}+\frac{21}{32} q_{4,0}{ }^{2}-3 / 4 q_{2,1} p_{3,0} \\
& +\left(1 / 8 q_{0,2}+3 / 8 p_{1,1}-1 / 4 q_{4,0}\right)^{2}+\left(1 / 8 q_{0,2}+3 / 8 p_{1,1}-1 / 4 q_{4,0}\right)\left(1 / 4 q_{0,2}+3 / 4 p_{1,1}\right. \\
& \left.\left.-1 / 2 q_{4,0}\right)\right)+q_{0,2}^{3} p_{3,0}-1 / 2 p_{3,0} p_{1,1}^{3}-2{p_{1,1}}^{2} p_{3,0} q_{0,2}-3 / 2 p_{1,1} p_{3,0} q_{0,2}^{2}-7 / 4 q_{2,1} q_{0,2}^{2} p_{1,1} \\
& -7 / 6 q_{2,1} q_{0,2} p_{1,1}{ }^{2}-5 / 6 q_{2,1} q_{0,2}{ }^{3}-1 / 4 q_{2,1} p_{1,1}{ }^{3}+\left(\frac{11}{12} q_{2,1} q_{0,2}{ }^{2}+3 / 2 q_{2,1} q_{0,2} p_{1,1}+\frac{7}{12} q_{2,1} p_{1,1}{ }^{2}\right. \\
& \left.-1 / 4 q_{0,2}^{2} p_{3,0}+2 p_{1,1} p_{3,0} q_{0,2}+5 / 4 p_{1,1}^{2} p_{3,0}\right)\left(1 / 2 q_{0,2}+3 / 2 p_{1,1}-q_{4,0}\right)+\left(3 p_{3,0}+q_{2,1}\right)\left(2 a_{4}\right. \\
& +2\left(1 / 8 q_{0,2}+3 / 8 p_{1,1}-1 / 4 q_{4,0}\right)\left(-\frac{3}{64} p_{1,1} q_{0,2}+\frac{23}{128} p_{1,1}{ }^{2}-\frac{5}{32} q_{4,0} p_{1,1}-\frac{1}{128} q_{0,2}^{2}\right. \\
& \left.\left.+1 / 32 q_{4,0} q_{0,2}+\frac{7}{32} q_{4,0}^{2}-1 / 4 q_{2,1} p_{3,0}\right)\right)+\left(-1 / 8 q_{0,2} p_{3,0}-\frac{11}{8} p_{1,1} p_{3,0}+9 / 4 p_{3,0} q_{4,0}\right. \\
& \left.+5 / 8 q_{2,1} q_{0,2}-1 / 8 q_{2,1} p_{1,1}+3 / 4 q_{2,1} q_{4,0}\right)\left(\frac{87}{64} p_{1,1} q_{0,2}+\frac{229}{128} p_{1,1}^{2}-\frac{79}{32} q_{4,0} p_{1,1}+\frac{29}{128} q_{0,2}^{2}\right. \\
& -\frac{29}{32} q_{4,0} q_{0,2}+\frac{21}{32} q_{4,0}^{2}+1 / 4 q_{2,1} p_{3,0}+\left(1 / 8 q_{0,2}+3 / 8 p_{1,1}-1 / 4 q_{4,0}\right)^{2}+\left(1 / 8 q_{0,2}+3 / 8 p_{1,1}\right. \\
& \left.-1 / 4 q_{4,0}\right)\left(1 / 4 q_{0,2}+3 / 4 p_{1,1}-1 / 2 q_{4,0}\right)+\left(-1 / 2 q_{0,2}-3 / 2 p_{1,1}+q_{4,0}\right)\left(1 / 2 q_{0,2}+3 / 2 p_{1,1}\right. \\
& \left.\left.-q_{4,0}\right)\right)+\left(-\frac{23}{32} p_{3,0} q_{4,0} q_{0,2}+\frac{35}{32} p_{3,0} q_{4,0} p_{1,1}-\frac{29}{32} q_{2,1} q_{4,0} q_{0,2}+1 / 32 q_{2,1} q_{4,0} p_{1,1}\right. \\
& +9 / 4 q_{2,1} p_{3,0}{ }^{2}-\frac{81}{32} p_{3,0} q_{4,0}{ }^{2}+3 / 4 q_{2,1}^{2} p_{3,0}-\frac{27}{32} q_{2,1} q_{4,0}{ }^{2}+\frac{23}{64} q_{2,1} q_{0,2} p_{1,1} \\
& \left.+\frac{5}{64} p_{1,1} p_{3,0} q_{0,2}-\frac{169}{384} q_{2,1} q_{0,2}^{2}-\frac{17}{384} q_{2,1} p_{1,1}^{2}+\frac{87}{128} q_{0,2}^{2} p_{3,0}-\frac{145}{128} p_{1,1}^{2} p_{3,0}\right) \\
& \left(-1 / 8 q_{0,2}-3 / 8 p_{1,1}+1 / 4 q_{4,0}\right)
\end{aligned}
$$




$$
\begin{aligned}
& E=\left(( - 3 p _ { 3 , 0 } - q _ { 2 , 1 } ) \left(\left(-5 / 4 p_{1,1} q_{0,2}^{2}-\frac{15}{8} p_{1,1}^{2} q_{0,2}-1 / 8 q_{0,2}{ }^{3}-3 / 4 p_{1,1}{ }^{3}+q_{0,2} p_{3,0}{ }^{2}+5 / 6 q_{4,0} q_{0,2}{ }^{2}\right.\right.\right. \\
& +3 q_{4,0} p_{1,1} q_{0,2}+\frac{13}{6} q_{4,0} p_{1,1}^{2}-3 / 2 q_{2,1} p_{3,0} p_{1,1}-3 / 2 q_{2,1} p_{3,0} q_{0,2} \\
& )\left(3 / 4 q_{0,2}+9 / 4 p_{1,1}-3 / 2 q_{4,0}\right)+\left(-1 / 2 q_{0,2}-3 / 2 p_{1,1}+q_{4,0}\right)\left(2 \left(-\frac{3}{32} p_{1,1} q_{0,2}\right.\right. \\
& +\frac{23}{64} p_{1,1}^{2}-\frac{5}{16} q_{4,0} p_{1,1}-\frac{1}{64} q_{0,2}^{2}+1 / 16 q_{4,0} q_{0,2} \\
& \left.+\frac{7}{16} q_{4,0}^{2}-1 / 2 q_{2,1} p_{3,0}+\left(1 / 8 q_{0,2}+3 / 8 p_{1,1}-1 / 4 q_{4,0}\right)^{2}\right)\left(1 / 4 q_{0,2}\right. \\
& \left.+3 / 4 p_{1,1}-1 / 2 q_{4,0}\right)+4 a_{4}+4\left(1 / 8 q_{0,2}+3 / 8 p_{1,1}-1 / 4 q_{4,0}\right)\left(-\frac{3}{64} p_{1,1} q_{0,2}\right. \\
& +\frac{23}{128} p_{1,1}^{2}-\frac{5}{32} q_{4,0} p_{1,1}-\frac{1}{128} q_{0,2}^{2}+1 / 32 q_{4,0} q_{0,2} \\
& \left.\left.+\frac{7}{32} q_{4,0}^{2}-1 / 4 q_{2,1} p_{3,0}\right)\right)+a_{4}\left(1 / 4 q_{0,2}+3 / 4 p_{1,1}-1 / 2 q_{4,0}\right) \\
& +\left(1 / 8 q_{0,2}+3 / 8 p_{1,1}-1 / 4 q_{4,0}\right)\left(2 a_{4}+2\left(1 / 8 q_{0,2}+3 / 8 p_{1,1}-1 / 4 q_{4,0}\right)\right. \\
& \left(-\frac{3}{64} p_{1,1} q_{0,2}+\frac{23}{128} p_{1,1}^{2}-\frac{5}{32} q_{4,0} p_{1,1}-\frac{1}{128} q_{0,2}^{2}\right. \\
& \left.\left.+1 / 32 q_{4,0} q_{0,2}+\frac{7}{32} q_{4,0}{ }^{2}-1 / 4 q_{2,1} p_{3,0}\right)\right)+2\left(1 / 8 q_{0,2}+3 / 8 p_{1,1}-1 / 4 q_{4,0}\right) a_{4} \\
& +\left(-\frac{3}{64} p_{1,1} q_{0,2}+\frac{23}{128} p_{1,1}^{2}-\frac{5}{32} q_{4,0} p_{1,1}-\frac{1}{128} q_{0,2}{ }^{2}\right. \\
& \left.+1 / 32 q_{4,0} q_{0,2}+\frac{7}{32} q_{4,0}^{2}-1 / 4 q_{2,1} p_{3,0}\right)^{2}+\left(-\frac{3}{64} p_{1,1} q_{0,2}\right. \\
& +\frac{23}{128} p_{1,1}^{2}-\frac{5}{32} q_{4,0} p_{1,1}-\frac{1}{128} q_{0,2}^{2} \\
& \left.+1 / 32 q_{4,0} q_{0,2}+\frac{7}{32} q_{4,0}{ }^{2}-1 / 4 q_{2,1} p_{3,0}\right)\left(-\frac{3}{32} p_{1,1} q_{0,2}+\frac{23}{64} p_{1,1}^{2}\right. \\
& -\frac{5}{16} q_{4,0} p_{1,1}-\frac{1}{64} q_{0,2}^{2}+1 / 16 q_{4,0} q_{0,2}+\frac{7}{16} q_{4,0}^{2} \\
& \left.-1 / 2 q_{2,1} p_{3,0}+\left(1 / 8 q_{0,2}+3 / 8 p_{1,1}-1 / 4 q_{4,0}\right)^{2}\right)+\frac{7}{120} q_{0,2}{ }^{4} \\
& +p_{1,1} q_{0,2}{ }^{3}+\frac{25}{12} p_{1,1}^{2} q_{0,2}^{2}-2 / 3 q_{4,0} q_{0,2}{ }^{3}-5 / 3 q_{4,0} p_{1,1}{ }^{3}+3 q_{2,1} p_{3,0} p_{1,1} q_{0,2} \\
& +5 / 3 q_{2,1} p_{3,0} q_{0,2}{ }^{2}+4 / 3 q_{2,1} p_{3,0} p_{1,1}{ }^{2}-13 / 3 q_{4,0} p_{1,1}{ }^{2} q_{0,2} \\
& -10 / 3 q_{4,0} p_{1,1} q_{0,2}{ }^{2}+\frac{43}{120} p_{1,1}^{4}+3 / 2 q_{0,2} p_{1,1}{ }^{3}-2 q_{0,2}{ }^{2} p_{3,0}{ }^{2} \\
& -q_{0,2} p_{3,0}^{2} p_{1,1}+\left(1 / 4 q_{0,2}^{2}-q_{4,0} q_{0,2}+5 / 4 p_{1,1}{ }^{2}+3 / 2 p_{1,1} q_{0,2}\right. \\
& \left.-2 q_{4,0} p_{1,1}+q_{2,1} p_{3,0}\right)\left(\left(1 / 8 q_{0,2}+3 / 8 p_{1,1}-1 / 4 q_{4,0}\right)\left(1 / 2 q_{0,2}+3 / 2 p_{1,1}-q_{4,0}\right)\right. \\
& -\frac{15}{64} p_{1,1} q_{0,2}+\frac{115}{128} p_{1,1}^{2}-\frac{25}{32} q_{4,0} p_{1,1}-\frac{5}{128} q_{0,2}^{2}+\frac{5}{32} q_{4,0} q_{0,2}+\frac{35}{32} q_{4,0}{ }^{2} \\
& \left.\left.-5 / 4 q_{2,1} p_{3,0}+2\left(1 / 8 q_{0,2}+3 / 8 p_{1,1}-1 / 4 q_{4,0}\right)^{2}+\left(1 / 4 q_{0,2}+3 / 4 p_{1,1}-1 / 2 q_{4,0}\right)^{2}\right)\right) \\
& +\left(\frac{11}{12} q_{2,1} q_{0,2}{ }^{2}+3 / 2 q_{2,1} q_{0,2} p_{1,1}+\frac{7}{12} q_{2,1} p_{1,1}{ }^{2}-1 / 4 q_{0,2}{ }^{2} p_{3,0}+2 p_{1,1} p_{3,0} q_{0,2}+5 / 4 p_{1,1}{ }^{2} p_{3,0}\right) \\
& \left(-3 / 16 p_{1,1} q_{0,2}+\frac{23}{32} p_{1,1}^{2}-5 / 8 q_{4,0} p_{1,1}-1 / 32 q_{0,2}^{2}+1 / 8 q_{4,0} q_{0,2}+\frac{7}{8} q_{4,0}^{2}\right. \\
& \left.-q_{2,1} p_{3,0}+2\left(1 / 8 q_{0,2}+3 / 8 p_{1,1}-1 / 4 q_{4,0}\right)^{2}+\left(1 / 4 q_{0,2}+3 / 4 p_{1,1}-1 / 2 q_{4,0}\right)^{2}\right)[\ldots]
\end{aligned}
$$




$$
\begin{aligned}
& E=[\ldots]+\left(-q_{0,2} p_{3,0}-2 p_{1,1} p_{3,0}-q_{2,1} q_{0,2}-q_{2,1} p_{1,1}\right)\left(( 1 / 8 q _ { 0 , 2 } + 3 / 8 p _ { 1 , 1 } - 1 / 4 q _ { 4 , 0 } ) \left(-\frac{3}{32} p_{1,1} q_{0,2}\right.\right. \\
& +\frac{23}{64} p_{1,1}^{2}-\frac{5}{16} q_{4,0} p_{1,1}-\frac{1}{64} q_{0,2}^{2}+1 / 16 q_{4,0} q_{0,2}+\frac{7}{16} q_{4,0}^{2}-1 / 2 q_{2,1} p_{3,0} \\
& \left.+\left(1 / 8 q_{0,2}+3 / 8 p_{1,1}-1 / 4 q_{4,0}\right)^{2}\right)+3 a_{4}+2\left(1 / 8 q_{0,2}+3 / 8 p_{1,1}-1 / 4 q_{4,0}\right) \\
& \left(-\frac{3}{64} p_{1,1} q_{0,2}+\frac{23}{128} p_{1,1}^{2}-\frac{5}{32} q_{4,0} p_{1,1}-\frac{1}{128} q_{0,2}^{2}+1 / 32 q_{4,0} q_{0,2}+\frac{7}{32} q_{4,0}^{2}\right. \\
& \left.-1 / 4 q_{2,1} p_{3,0}\right)+\left(-\frac{3}{64} p_{1,1} q_{0,2}+\frac{23}{128} p_{1,1}^{2}-\frac{5}{32} q_{4,0} p_{1,1}-\frac{1}{128} q_{0,2}{ }^{2}\right. \\
& \left.\left.+1 / 32 q_{4,0} q_{0,2}+\frac{7}{32} q_{4,0}^{2}-1 / 4 q_{2,1} p_{3,0}\right)\left(1 / 4 q_{0,2}+3 / 4 p_{1,1}-1 / 2 q_{4,0}\right)\right)+\left(3 p_{3,0}+q_{2,1}\right)\left(2 \left(1 / 8 q_{0,2}\right.\right. \\
& \left.+3 / 8 p_{1,1}-1 / 4 q_{4,0}\right) a_{4}+\left(-\frac{3}{64} p_{1,1} q_{0,2}+\frac{23}{128} p_{1,1}^{2}-\frac{5}{32} q_{4,0} p_{1,1}-\frac{1}{128} q_{0,2}^{2}\right. \\
& \left.\left.+1 / 32 q_{4,0} q_{0,2}+\frac{7}{32} q_{4,0}^{2}-1 / 4 q_{2,1} p_{3,0}\right)^{2}\right)+\left(q_{0,2}{ }^{3} p_{3,0}\right. \\
& -1 / 2 p_{3,0} p_{1,1}{ }^{3}-2 p_{1,1}^{2} p_{3,0} q_{0,2}-3 / 2 p_{1,1} p_{3,0} q_{0,2}^{2}-7 / 4 q_{2,1} q_{0,2}{ }^{2} p_{1,1} \\
& \left.-7 / 6 q_{2,1} q_{0,2} p_{1,1}{ }^{2}-5 / 6 q_{2,1} q_{0,2}{ }^{3}-1 / 4 q_{2,1} p_{1,1}{ }^{3}\right)\left(5 / 8 q_{0,2}+\frac{15}{8} p_{1,1}-5 / 4 q_{4,0}\right) \\
& +\frac{137}{180} q_{2,1} q_{0,2}^{4}+\frac{7}{40} p_{3,0} p_{1,1}^{4}-\frac{22}{15} p_{3,0} q_{0,2}{ }^{4} \\
& +\frac{31}{360} q_{2,1} p_{1,1}^{4}+\frac{55}{24} p_{1,1}^{2} p_{3,0} q_{0,2}^{2}+5 / 8 q_{2,1} q_{0,2} p_{1,1}^{3} \\
& +\frac{11}{12} p_{1,1} p_{3,0} q_{0,2}{ }^{3}+\frac{13}{12} q_{0,2} p_{3,0} p_{1,1}^{3}+\frac{119}{72} q_{2,1} q_{0,2}^{2} p_{1,1}^{2} \\
& +\frac{15}{8} q_{2,1} q_{0,2}{ }^{3} p_{1,1}+\left(-1 / 8 q_{0,2} p_{3,0}-\frac{11}{8} p_{1,1} p_{3,0}+9 / 4 p_{3,0} q_{4,0}\right. \\
& \left.+5 / 8 q_{2,1} q_{0,2}-1 / 8 q_{2,1} p_{1,1}+3 / 4 q_{2,1} q_{4,0}\right)\left(( - 1 / 2 q _ { 0 , 2 } - 3 / 2 p _ { 1 , 1 } + q _ { 4 , 0 } ) \left(-3 / 16 p_{1,1} q_{0,2}+\frac{23}{32} p_{1,1}^{2}\right.\right. \\
& -5 / 8 q_{4,0} p_{1,1}-1 / 32 q_{0,2}^{2}+1 / 8 q_{4,0} q_{0,2}+\frac{7}{8} q_{4,0}^{2}-q_{2,1} p_{3,0} \\
& \left.+2\left(1 / 8 q_{0,2}+3 / 8 p_{1,1}-1 / 4 q_{4,0}\right)^{2}+\left(1 / 4 q_{0,2}+3 / 4 p_{1,1}-1 / 2 q_{4,0}\right)^{2}\right)+\left(1 / 8 q_{0,2}+3 / 8 p_{1,1}\right. \\
& \left.-1 / 4 q_{4,0}\right)\left(-\frac{3}{32} p_{1,1} q_{0,2}+\frac{23}{64} p_{1,1}^{2}-\frac{5}{16} q_{4,0} p_{1,1}\right. \\
& -\frac{1}{64} q_{0,2}^{2}+1 / 16 q_{4,0} q_{0,2}+\frac{7}{16} q_{4,0}^{2}-1 / 2 q_{2,1} p_{3,0} \\
& \left.+\left(1 / 8 q_{0,2}+3 / 8 p_{1,1}-1 / 4 q_{4,0}\right)^{2}\right)+3 a_{4}+2\left(1 / 8 q_{0,2}+3 / 8 p_{1,1}-1 / 4 q_{4,0}\right)\left(-\frac{3}{64} p_{1,1} q_{0,2}\right. \\
& +\frac{23}{128} p_{1,1}^{2}-\frac{5}{32} q_{4,0} p_{1,1}-\frac{1}{128} q_{0,2}^{2}+1 / 32 q_{4,0} q_{0,2}+\frac{7}{32} q_{4,0}^{2} \\
& \left.-1 / 4 q_{2,1} p_{3,0}\right)+\left(-\frac{3}{64} p_{1,1} q_{0,2}+\frac{23}{128} p_{1,1}{ }^{2}-\frac{5}{32} q_{4,0} p_{1,1}-\frac{1}{128} q_{0,2}{ }^{2}\right. \\
& \left.+1 / 32 q_{4,0} q_{0,2}+\frac{7}{32} q_{4,0}^{2}-1 / 4 q_{2,1} p_{3,0}\right)\left(1 / 4 q_{0,2}+3 / 4 p_{1,1}-1 / 2 q_{4,0}\right)+\left(1 / 4 q_{0,2}{ }^{2}-q_{4,0} q_{0,2}\right. \\
& \left.+5 / 4 p_{1,1}{ }^{2}+3 / 2 p_{1,1} q_{0,2}-2 q_{4,0} p_{1,1}+q_{2,1} p_{3,0}\right)\left(5 / 8 q_{0,2}+\frac{15}{8} p_{1,1}-5 / 4 q_{4,0}\right) \\
& -5 / 4 p_{1,1} q_{0,2}{ }^{2}-\frac{15}{8} p_{1,1}{ }^{2} q_{0,2}-1 / 8 q_{0,2}{ }^{3}-3 / 4 p_{1,1}{ }^{3}+q_{0,2} p_{3,0}{ }^{2}+5 / 6 q_{4,0} q_{0,2}{ }^{2} \\
& \left.+3 q_{4,0} p_{1,1} q_{0,2}+\frac{13}{6} q_{4,0} p_{1,1}^{2}-3 / 2 q_{2,1} p_{3,0} p_{1,1}-3 / 2 q_{2,1} p_{3,0} q_{0,2}\right)[\ldots]
\end{aligned}
$$




$$
\begin{aligned}
& E=[\ldots]+\left(-\frac{23}{32} p_{3,0} q_{4,0} q_{0,2}+\frac{35}{32} p_{3,0} q_{4,0} p_{1,1}-\frac{29}{32} q_{2,1} q_{4,0} q_{0,2}\right. \\
& +1 / 32 q_{2,1} q_{4,0} p_{1,1}+9 / 4 q_{2,1} p_{3,0}{ }^{2}-\frac{81}{32} p_{3,0} q_{4,0}^{2}+3 / 4 q_{2,1}^{2} p_{3,0} \\
& -\frac{27}{32} q_{2,1} q_{4,0}^{2}+\frac{23}{64} q_{2,1} q_{0,2} p_{1,1}+\frac{5}{64} p_{1,1} p_{3,0} q_{0,2} \\
& -\frac{169}{384} q_{2,1} q_{0,2}^{2}-\frac{17}{384} q_{2,1} p_{1,1}^{2}+\frac{87}{128} q_{0,2}^{2} p_{3,0}-\frac{145}{128} p_{1,1}^{2} p_{3,0} \\
& )\left(\frac{87}{64} p_{1,1} q_{0,2}+\frac{229}{128} p_{1,1}^{2}-\frac{79}{32} q_{4,0} p_{1,1}+\frac{29}{128} q_{0,2}{ }^{2}\right. \\
& -\frac{29}{32} q_{4,0} q_{0,2}+\frac{21}{32} q_{4,0}^{2}+1 / 4 q_{2,1} p_{3,0}+\left(1 / 8 q_{0,2}+3 / 8 p_{1,1}-1 / 4 q_{4,0}\right)^{2} \\
& +\left(1 / 8 q_{0,2}+3 / 8 p_{1,1}-1 / 4 q_{4,0}\right)\left(1 / 4 q_{0,2}+3 / 4 p_{1,1}-1 / 2 q_{4,0}\right)+\left(-1 / 2 q_{0,2}-3 / 2 p_{1,1}+q_{4,0}\right) \\
& \left.\left(1 / 2 q_{0,2}+3 / 2 p_{1,1}-q_{4,0}\right)\right)+\left(-\frac{143}{256} q_{2,1} q_{0,2}^{2} p_{1,1}+\frac{265}{768} q_{2,1} q_{0,2} p_{1,1}{ }^{2}\right. \\
& +\frac{265}{256} p_{1,1}^{2} p_{3,0} q_{0,2}-\frac{15}{16} q_{2,1} p_{3,0}^{2} q_{0,2}-\frac{37}{64} q_{2,1} q_{4,0} p_{1,1} q_{0,2} \\
& +\frac{47}{384} q_{2,1} q_{4,0} p_{1,1}^{2}-\frac{71}{64} p_{3,0} q_{4,0} p_{1,1} q_{0,2}-\frac{49}{128} p_{3,0} q_{4,0} q_{0,2}{ }^{2} \\
& +\frac{43}{32} q_{2,1} q_{4,0}{ }^{3}+q_{2,1} a_{4}+\frac{129}{32} p_{3,0} q_{4,0}{ }^{3}+\frac{243}{256} p_{1,1} p_{3,0} q_{0,2}{ }^{2} \\
& +\frac{149}{64} p_{3,0} q_{0,2} q_{4,0}^{2}+\frac{191}{128} p_{3,0} q_{4,0} p_{1,1}^{2}-\frac{89}{64} p_{3,0} p_{1,1} q_{4,0}{ }^{2} \\
& -\frac{3}{64} q_{2,1} p_{1,1} q_{4,0}{ }^{2}-\frac{311}{256} p_{3,0} p_{1,1}{ }^{3}+\frac{251}{768} q_{2,1} q_{0,2}{ }^{3} \\
& -\frac{29}{256} q_{2,1} p_{1,1}^{3}+3 q_{0,2} p_{3,0}{ }^{3}+3 p_{3,0} a_{4}-\frac{57}{8} q_{4,0} q_{2,1} p_{3,0}{ }^{2} \\
& +\frac{67}{16} q_{2,1} p_{3,0}^{2} p_{1,1}-\frac{197}{256} q_{0,2}^{3} p_{3,0}+\frac{103}{64} q_{2,1} q_{0,2} q_{4,0}^{2} \\
& +\frac{17}{16} q_{2,1}^{2} p_{3,0} p_{1,1}-\frac{19}{8} q_{4,0} q_{2,1}^{2} p_{3,0}-\frac{21}{16} q_{2,1}^{2} p_{3,0} q_{0,2} \\
& \left.+\frac{367}{384} q_{2,1} q_{4,0} q_{0,2}{ }^{2}\right)\left(-1 / 8 q_{0,2}-3 / 8 p_{1,1}+1 / 4 q_{4,0}\right) x^{6}
\end{aligned}
$$




\section{Índice Remissivo}

Aplicação de Poincaré, 45

Blow-up

(m,n)-quasehomogêneo, 32

direcional, 25,28

polar, 22,28

Centro, 8

Centro linear, 8

Constante

de Lyapunov, 55, 61

de Lyapunov generalizada, 82, 83, 88

coordenadas $(\mathrm{m}, \mathrm{n})$-quasehomogêneas, 33

Estabilidade

assintótica, 58

de Lyapunov, 57

Foco, 8

Foco fraco, 9

Função

de Lyapunov, 58

sucessão, 49

Índice de campo vetorial planar, 74

Ponto singular degenerado elementar, 8

degenerado linearmente nulo, 8

hiperbólico, 12

monodrômico, 8

não degenerado, 8

nilpotente, 8

Problema

da estabilidade, 67, 84, 105

da monodromia, 67, 72, 104

do centro-foco, 37, 71

\section{Teorema}

centro de Lyapunov, 61

da estabilidade, 88

da monodromia , 73

das formas normais, 19

de Bautin, 63

de Hartman-Grobman, 12

do centro linear, 98

do centro nilpotente, 98

do fluxo tubular, 11 